\title{
Fingerprint of Tea
}

Citation for published version (APA):

Einöther, S. J. L. (2016). Fingerprint of Tea: acute mental benefits of tea consumption. [Doctoral Thesis, Maastricht University]. Universitaire Pers Maastricht. https://doi.org/10.26481/dis.20160511se

\section{Document status and date:}

Published: 01/01/2016

DOI:

10.26481/dis.20160511se

Document Version:

Publisher's PDF, also known as Version of record

\section{Please check the document version of this publication:}

- A submitted manuscript is the version of the article upon submission and before peer-review. There can be important differences between the submitted version and the official published version of record.

People interested in the research are advised to contact the author for the final version of the publication, or visit the DOI to the publisher's website.

- The final author version and the galley proof are versions of the publication after peer review.

- The final published version features the final layout of the paper including the volume, issue and page numbers.

Link to publication

\footnotetext{
General rights rights.

- You may freely distribute the URL identifying the publication in the public portal. please follow below link for the End User Agreement:

www.umlib.nl/taverne-license

Take down policy

If you believe that this document breaches copyright please contact us at:

repository@maastrichtuniversity.nl

providing details and we will investigate your claim.
}

Copyright and moral rights for the publications made accessible in the public portal are retained by the authors and/or other copyright owners and it is a condition of accessing publications that users recognise and abide by the legal requirements associated with these

- Users may download and print one copy of any publication from the public portal for the purpose of private study or research.

- You may not further distribute the material or use it for any profit-making activity or commercial gain

If the publication is distributed under the terms of Article $25 \mathrm{fa}$ of the Dutch Copyright Act, indicated by the "Taverne" license above, 


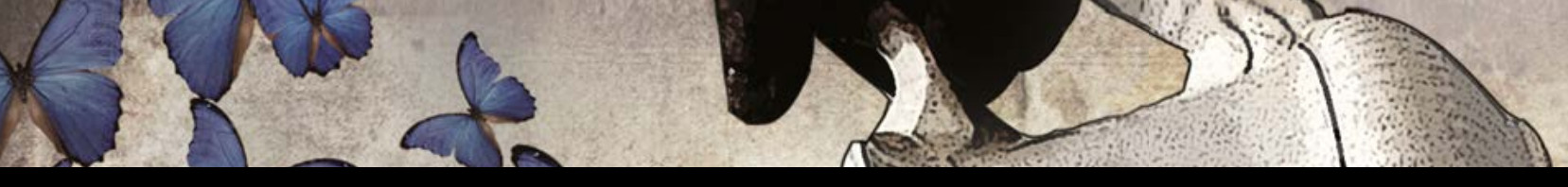

\section{Fingerprint of Tea}

Acute mental benefits of tea consumption

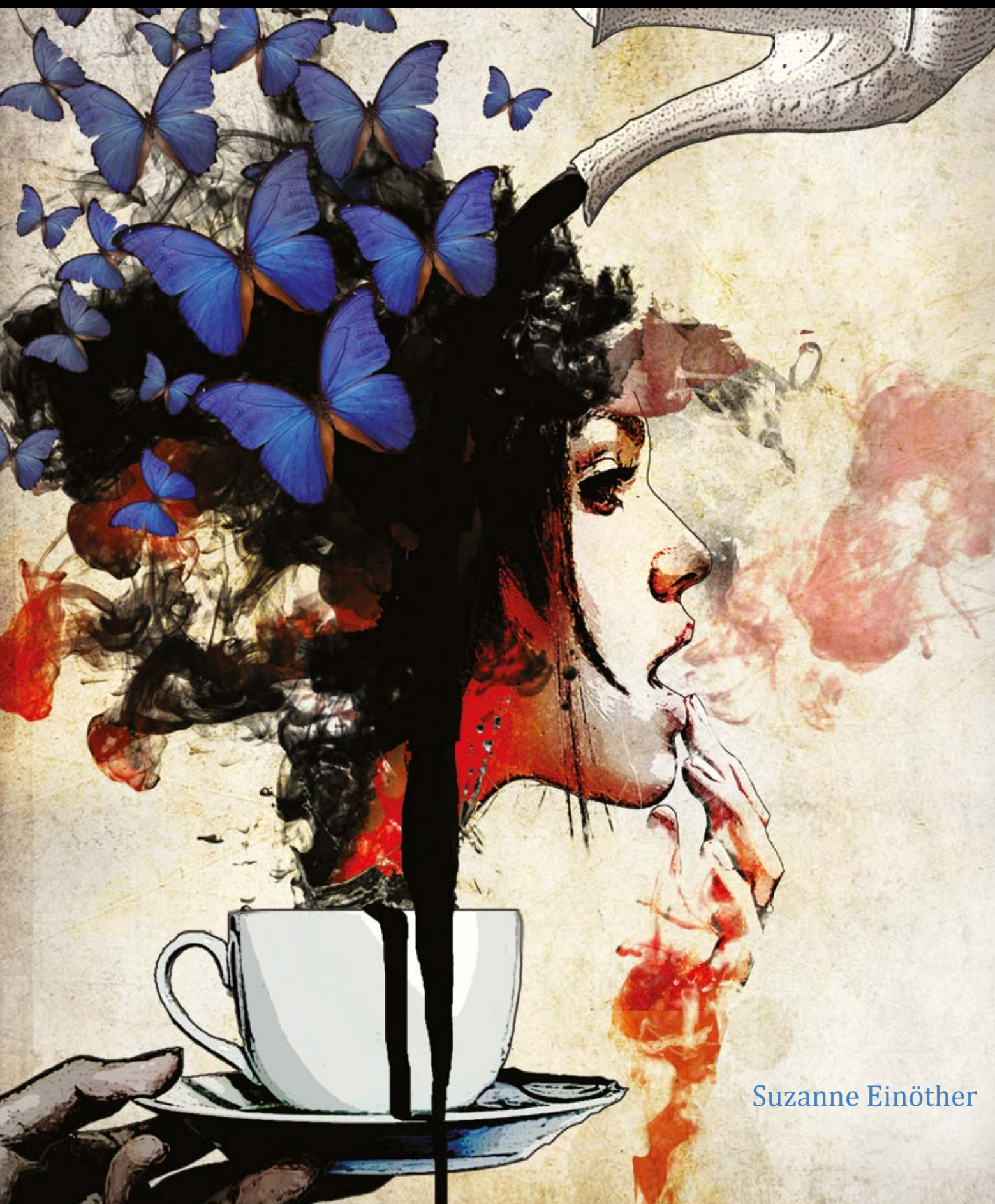


Illustration: “Cup Tea” by Stefano Messina

Publisher: Datawyse / Universitaire Pers Maastricht ISBN: 9789461595539 


\section{Fingerprint of Tea}

\section{Acute mental benefits of tea consumption}

\section{PROEFSCHRIFT}

ter verkrijging van de graad van doctor aan de Universiteit Maastricht, op gezag van de Rector Magnificus, Prof. dr. L.L.G. Soete, volgens het besluit van het College van Decanen, in het openbaar te verdedigen op woensdag 11 mei 2016, om 14.00 uur

door

\section{Suzanne Einöther}




\section{Promotiecommissie:}

Promotor:

Prof. dr. J. G. Ramaekers

Copromotor:

Dr. T. Giesbrecht

\section{Beoordelingscommissie}

Prof. dr. W.J. Riedel (voorzitter)

Prof. dr. L. Dye (University of Leeds)

Dr. P.W.M. Van Gerven

Prof. dr. M.M. Lorist (Rijksuniversiteit Groningen)

Prof. dr. C.R. Markus 


\section{Content}

1 General Introduction $\quad 7$

2 Caffeine as an attention enhancer: Reviewing existing assumptions 19

3 L-theanine \& caffeine improve task switching but not intersensory attention or subjective alertness

$4 \quad$ Acute effects of tea consumption on attention and mood 71

5 Investigating the effects of tea, water and a positive affect induction on mood and creativity

6 Infusing Pleasure: Mood effects of the consumption of a single cup of tea

$7 \quad$ General Discussion

References

Summary

Samenvatting

Valorisation Addendum

Dank | Thanks

Publications 

"My dear if you could give me a cup of tea to clear my muddle of a head, I should better understand your affairs "

- Charles Dickens (1812-1870), British novelist

"If you are cold, tea will warm you; if you are too heated, it will cool you; If you are depressed, it will cheer you; if you are excited, it will calm you. ", - William Gladstone (1809-1898), politician and former Prime Minister

Tea, the aromatic hot beverage prepared from the leaves and buds of the tropical Camellia Sinensis plant, is one of the most widely consumed beverages in the world. In fact, tea is the second most consumed beverage, only surpassed by water. According to market research data from Euromonitor in $2014^{1}$, China has the highest overall consumption of tea (excluding herbal teas) with over 700.000 kilograms of tea consumed per year, while the highest per capita tea consumption is seen in Turkey, at around $3 \mathrm{~kg}$ consumed annually per capita. In the United Kingdom, also traditionally a tea-loving nation, just under $2 \mathrm{~kg}$ tea is consumed annually per capita, while the Dutch consume $0,777 \mathrm{~kg}$ annually per capita. The Chinese and the Turks typically use six grams of tea per cup, but the Chinese continuously add hot water to the same tea leaves throughout the day. The tea bags typically used by the British and Dutch tea consumers normally include two grams of tea. Either way, this adds up to a lot of cups of tea being consumed daily across the world.

There are four varieties of tea, all prepared from the leaves of the Camellia Sinensis plant, i.e. black, green, white and oolong tea. The most consumed varieties are black and green tea. Typically, black tea is consumed more frequently in Western countries, the Middle East and West Asia (e.g. India and Sri Lanka), whereas green tea is consumed more frequently in East Asia (e.g. China and Japan). Tea varieties differ due

\footnotetext{
${ }^{1}$ Sources: http://qz.com/168690/where-the-worlds-biggest-tea-drinkers-are and http://www.worldteanews.com/news/per-capita-tea, retrieved October 13, 2015
} 
to differences in the leaf processing, specifically by the degree of fermentation or oxidation of the leaves. This affects the teas sensorial properties, such as aroma, taste, and colour, but also some tea compounds, such as flavonoids. The research in this thesis mainly focuses on black tea. Compared to other varieties, black tea is the most oxidized, hence its darker colour and stronger aroma and taste.

Why do people drink tea? An important reason for its popularity, besides aroma, taste and habit, is the acclaimed stimulating effect of tea. Tea originates in China, where it has been consumed for its alleged medicinal and stimulating properties since the Shang dynasty (1766-1122 BC) (Heiss \& Heiss, 2007). An early medicinal text $\left(3^{\text {rd }}\right.$ century $\mathrm{AD})$ by the Han dynasty surgeon Hua Tuo stated that "to drink bitter tea constantly makes one think better" (Chen, 2003). Also, Buddhist monks commonly consumed tea to facilitate their mediation practice (Macfarlane \& Macfarlane, 2004). During the $17^{\text {th }}$ century, tea became popular in the West via British traders. Initially a luxury item for the rich, reserved for special occasions such as "afternoon tea", tea finally became a common beverage for all levels of society by the late $19^{\text {th }}$ century. Notably, this was the time from which the quotes on the previous page stem.

Thus, historically and traditionally, tea has been acclaimed to provide a variety of mental benefits. More recent consumer beliefs include feelings of relaxation, but also feeling energized and having clarity of mind (Graham, 1992; Shimbo, Nakamura, Jing, Kizuki, Seino et al., 2005). These benefits are highly relevant today, in this day and age of high demand, where people constantly seek for the things that make them feel and perform better. Nevertheless, until a decade ago, the alleged mental effects of tea were solely based on anecdotal evidence. The aim of this thesis was to further investigate the acute mental benefits of the consumption of tea. 


\section{MENTAL BENEFITS OF TEA CONSUMPTION}

In this section, I will briefly outline the research to date on the mental benefits of black tea. Notably, this research has focused on two types of evidence. Firstly, epidemiological studies have aimed to identify the neuro-protective effects of habitual tea consumption over a longer period of time on mental health and wellbeing, via lasting effects on brain structure and function. Secondly, randomised placebo-controlled intervention studies aimed to identify the acute modulation of mental performance and mood in healthy subjects shortly after tea consumption.

Epidemiological research has linked frequent tea consumption (including green, black, and oolong varieties) to long-term mental benefits (Song, Xu, Liu, \& Feng, 2012). Cross-sectional studies have shown positive correlations between tea intake and higher cognitive performance or lower prevalence of cognitive impairment, in European (e.g. Nurk, Refsum, Drevon, Tell, Nygaard et al., 2009) and Asian (e.g. Feng, Gwee, Kua, \& Ng, 2010; Ng, Feng, Niti, Kua, \& Yap, 2008) elderly populations, and well as lower prevalence of depressive symptoms in the latter (Feng, Li, Kua, Lee, Yap et al., 2012; Pham, Nanri, Kochi, Kuwahara, Tsuruoka et al., 2014). Moreover, longitudinal studies have found associations with reduced cognitive decline for tea (e.g. Arab, Biggs, O'Meara, Longstreth, Crane et al., 2011) and caffeine consumption (including tea and coffee) (e.g. Van Boxtel, Schmitt, Bosma, \& Jolles, 2003). Future studies need to confirm findings and demonstrate the mechanisms underlying potential neuro-protective effects (Panza, Solfrizzi, Barulli, Bonfiglio, Guerra et al., 2015). However, these studies do give credibility to the idea that tea consumption can have lasting effects on mental well-being and performance, particularly in elderly or impaired populations (Song et al., 2012).

More importantly to the topic of this thesis, a smaller number of randomised placebo-controlled intervention studies have linked black tea consumption, and the consumption of its ingredients, to acute mental benefits (Einöther \& Martens, 2013). Black tea contains a large number of constituents. Its acute mental benefits, however, are generally attributed to two ingredients, the methylxanthine caffeine and the amino acid theanine (Bryan, 2008). A typical serving of black tea $(200 \mathrm{~mL})$ naturally contains ap- 
proximately 35 to $61 \mathrm{mg}$ of caffeine and 4.5 to $22.5 \mathrm{mg}$ of theanine. While caffeine can be found in a variety of other foods (e.g., coffee, chocolate), theanine is solely present in tea from the Camellia Sinensis plant, and in the edible bay boletes mushroom Xerocomus badius. Therefore, the combination of theanine and caffeine is unique to tea. As other chapters in this thesis will address the mechanism of action of caffeine (Chapter 2, 3 and 4) and theanine (Chapter 3 and 4), this introduction will not address these in detail All the individual studies investigating the acute mental effects of tea and theanine and caffeine consumption will also be addressed later in this thesis (Chapter 4) in more detail. In this introduction, I therefore highlight two key pieces of research: two studies on tea, and a recent review of studies on tea ingredients theanine and caffeine. Two studies investigated the effects of black tea consumption compared to a placebo tea on attention and mood (De Bruin, Rowson, Van Buren, Rycroft, \& Owen, 2011). Findings showed that consumption of two (study 1) or three cups (study 2) of tea improved attention. After consuming the tea, participants also reported feeling more alert. Moreover, the meta-analysis on theanine and caffeine effects (Camfield, Stough, Farrimond, \& Scholey, 2014) confirmed that theanine and caffeine consumed in combination increased attention and feelings alertness up to two hours after consumption. It also suggested that calmness ratings decreased as the caffeine dose increased, and increased as the theanine dose increased. Notably, insufficient data were available to conduct a metaanalysis on the green tea polyphenol epigallocatechin gallate (EGCG). While EGCG has been associated with neuro-protective effects (Scholey, Downey, Ciorciari, Pipingas, Nolidin et al., 2012), acute mental benefits have not been found (Wightman, Haskell, Forster, Veasey, \& Kennedy, 2012). Further investigation of EGCG was therefore not part of the current thesis. 


\section{A FRAMEWORK}

The objective of this thesis is to further investigate the acute mental benefits of the consumption of tea (and tea ingredients). These acute mental benefits can be characterized in terms of two benefit dimensions, namely cognitive and affective, and along three post-consumption stages, namely immediately after consumption (i.e. after 5 to 10 minutes), shortly after consumption (i.e. after 10 to 30 minutes), and 30 minutes or more after consumption (see Fig. 1). In the sections below, I explain the rationale for this framework.

Fig. 1 Framework

Immediately after

(5-10 min)
Shortly after

(10-30 min)
Over time

(30+ min)

\section{Cognitive benefits}

Affective benefits

\section{Benefit dimensions}

\section{Cognitive}

Cognitive effects of tea consumption are often established in terms of improved attention. The cognitive process of attention is vital to everyday functioning, because the human brain has limited information processing capacity. The concept of attention refers to a number of related cognitive processes, such as selective attention (i.e. the ability to focus on relevant information while ignoring the irrelevant), divided attention (i.e. the ability to perform multiple tasks concurrently), sustained attention (i.e. the ability to maintain focused for a prolonged period), and executive control (i.e. the ability to inhibit inappropriate responses) (Strayer \& Drews, 2007). 
With regard to tea, improvements in selected and divided attention, and executive control have been consistently demonstrated, specifically after 40 to 100 minutes (De Bruin et al., 2011). Typically, these effects are ascribed to the tea ingredients theanine and caffeine. Changes in other cognitive domains, such as memory or reasoning, have not yet been investigated in relation to acute effects of tea consumption.

\section{Affective}

Affective benefits refer to (changes in) mood. The concept of "mood" refers to two different but related concepts. Mood is considered a low-intensity but enduring affective state (Lucas, Diener, \& Larsen, 2009). Often however the term mood also refers to "emotions", which are relatively brief but intense affective responses that result from a specific event (Mesquita \& Frijda, 1992), Mood effects can manifest themselves in terms of subjective feelings (i.e. self-reported mood), but also in terms of physiological responses and changes in behaviour. Due to theoretical controversies, mood is classified differently in different frameworks, either reducing affective states to two dimensions such as arousal and valence or positive and negative affect (Russell \& Carroll, 1999; Watson, Clark, \& Tellegen, 1988), or viewing each affective state as a specific or discrete emotion, i.e. disgust, happiness, surprise etcetera (Ekman, 1992).

Research to date has mainly focused on feelings of arousal or alertness, particularly due to tea ingredients theanine and caffeine, and occurring during the last postconsumption stage (Einöther \& Martens, 2013). It is less clear whether tea also has effects on pleasure or valence, or whether effects of tea consumption can also occur rather immediately after consumption. Interestingly, recent studies have suggested a role for aroma volatiles from green tea or so-called "green odours", such as those from the tea plant, in stress reduction and relaxation (Murao, Yoto, \& Yokogoshi, 2013).

\section{Post consumption stages}

Acute mental benefits of tea consumption can also be characterized along three postconsumption stages, i.e., immediately after, shortly after, and 30 minutes or more after consumption. The majority of the research to date has assessed potential effects acutely 
from around 30 to 45 minutes after consumption, up until about 2 hours later. Typically, this time frame is chosen on the basis of the known mechanism of action of the tea ingredients caffeine and theanine, and relies on the time required for absorption and transport of these psychoactive ingredients to the brain (caffeine: Magkos \& Sidossis, 2005; theanine: Van der Pijl, Chen, \& Mulder, 2010). Thus, pharmacological effects of tea ingredients would not occur during or immediately/shortly after consumption.

Other aspects of tea may however contribute to more immediate benefits. Tea is unique from other beverages because of its preparation procedure of pouring hot water on a bag containing tea leaves, the temperature at which it is drunk, other sensory properties such as aroma and flavour, its astringency, and, in some cultures, ritualistic behaviours associated with its consumption (Dufresne \& Farnworth, 2001). Interestingly, research indeed points to a number of possible sources for immediate food-related emotions, including sensory attributes, experienced consequences, anticipated consequences, personal or cultural meanings (Desmet \& Schifferstein, 2008). The tea consumption experience also includes a combination of these emotion sources, which may affect the consumption experience and its subsequent effects.

Additionally, physiological effects of tea consumption seem to occur in each of the three stages (Aspen \& Quinlan, 1998; Quinlan, Lane, \& Aspinall, 1997; Quinlan, Lane, Moore, Aspen, Rycroft et al., 2000): small and transient increases in heart rate and more pronounced increases in skin conductance, likely due to sensorial properties (immediate), increases in skin temperature and skin conductance, due to its temperature (shortly after) and increases in blood pressure and skin conductance, due to caffeine (30 to $60 \mathrm{~min}$ post-consumption). This makes it probable that mental effects occur in each of the three post consumption stages, as these physiological changes may cause people to feel and perform differently. 


\section{THESIS OVERVIEW}

Following the framework presented in the previous section, the objective of this thesis is to further investigate the acute mental benefits of the consumption of tea (and tea ingredients), using the framework described of two benefit dimensions (i.e., cognitive and affective) and three post-consumption stages (i.e., immediately after, shortly after, and after 30 minutes or more). Fig. 2 shows a schematic overview of the content of this thesis in the light of the framework, and in the section below I will outline the different chapters.

\section{Fig. 2 Schematic overview of thesis using the framework}

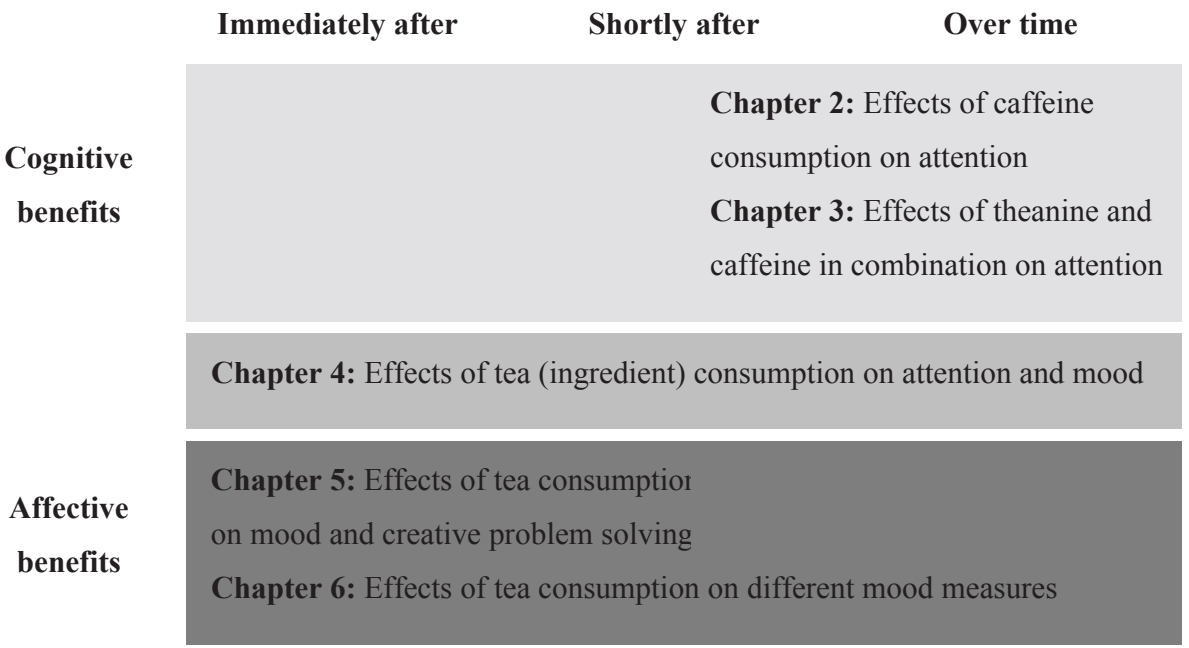

Chapter 2 provides a detailed review of the effects of caffeine on attention performance, with a number of specific hypotheses. We hypothesised that caffeine has a general effect across attention domains rather than on specific attention domains. We reviewed effects of caffeine on performance on both simple and complex attention tasks, as well as its effect on tasks related to each of the three attention networks (i.e. alerting, orienting, executive control; Posner \& Dehaene, 1994). We hypothesised that 
performance effects are more pronounced for higher levels of caffeine (i.e. doseproportional). Moreover, we reviewed studies that investigated the influence of habitual caffeine consumption on attention performance. We also addressed the issue of withdrawal. Often in caffeine studies, participants are deprived of their habitual caffeine intake for a certain period preceding the study. We hypothesized that effects of caffeine consumption are not influenced by habitual caffeine use or withdrawal. In total, we reviewed 66 peer-reviewed, double blind placebo controlled studies that assessed acute effects of caffeine on attention in healthy adult volunteers.

Chapter 3 presents a study addressing the effects of an iced tea containing a combination of theanine and caffeine on measures of attention and subjective alertness. The aim of this study was to provide further insights on the combination of theanine and caffeine, which is unique to tea. Specifically, the study aimed to replicate effects of a theanine/ caffeine combination on a switch task (Owen et al., 2008). Furthermore, we wanted to assess the specificity of this effect by employing a different attention task. While the switch task involved switching attention between visual stimuli only and required participants to attend each visual stimulus and switch between two rules for their response, our second task involved both visual and auditory stimuli and required participants to switch attention between the visual and auditory stimuli. In line with previous studies, the dose of theanine relative to caffeine in the iced tea was increased in order to amplify possible effects of theanine.

Chapter 4 consists of a review that summarizes research to date on the acute mental effects of tea, with specific reference to its ingredients theanine and caffeine, on attention and mood. Included studies were required to report data on at least one measure of attention or mood in relation to consumption of tea assessed in a sample of healthy adults. This resulted in four studies on effects of tea consumption on attention, nine on mood effects, and six on effects of a combination of caffeine and theanine, mainly on cognition. In addition, it briefly covers the research to date on tea, theanine and caffeine on relevant outcomes outside the laboratory. 
Chapter 5 pertains to an investigation of the acute effects of tea, water and a positive affect induction on mood and creativity immediately after consumption. As seen in Chapter 4, studies to date focused largely on effects of tea consumption from 30 minutes post consumption based on the known mechanism of action for theanine and caffeine. As such, they have neglected potential effects that tea consumption may have during and/or immediately or shortly after consumption due to other mechanisms, such as the pleasantness of the tea consumption experience. This may include the preparation of the tea, its sensory properties (i.e. smell, taste, mouth feel, and appearance) before, during and after consumption, and even simply providing a break from other activities. The primary aim of this study was to assess the immediate mood effects of tea preparation and consumption. Moreover, as improvements in mood have been related to improved creativity task performance, the secondary aim of this study was to assess the effect of tea preparation and consumption on creative problem solving, as a result of a potential mood change. Tea consumption was compared to water, and a validated positive affect induction in parallel conditions.

In Chapter 6, the immediate benefits of tea on aspects of mood over time are explored in more detail using different mood frameworks, such as the bipolar framework which differentiates between valence and arousal (Russell, 1989), as well as the dimensional framework which represents specific discrete emotions (Ekman, 1992). This study assessed effects of tea consumption on valence and arousal immediately after consumption (primary hypothesis) and up to 60 minutes post-consumption (secondary hypothesis). Moreover the study explored effect on self-reported discrete emotions, both immediately and over time up to 60 minutes post-consumption, and on a selection of more implicit (as compared to the above explicit) mood tests. The study employed a parallel design with three conditions: tea, water, and a placebo tea of coloured and teaflavoured water.

Finally, in Chapter 7 I will integrate and discuss findings, while addressing implications of and gaps in the research to date, as well as opportunities for future research. 



\section{Caffeine as an attention enhancer: \\ Reviewing existing assumptions}

Einöther, S.J.L., \& Giesbrecht, T. (2013). Caffeine as an attention enhancer: reviewing existing assumptions. Psychopharmacology, 225, 251-274. 


\section{ABSTRACT}

Despite the large number of studies on the behavioural effects of caffeine, an unequivocal conclusion has not been reached. In this review, we seek to disentangle a number of questions. Whereas there is a general consensus that caffeine can improve performance on simple tasks, it is not clear whether complex tasks are also affected, or if caffeine affects performance of the attention networks (alerting, orienting, executive control). Other questions being raised in this review are whether effects are more pronounced for higher levels of caffeine, are influenced by habitual caffeine use, and whether these effects are due to withdrawal reversal. This literature review summarises the results of double blind placebo controlled studies that assessed acute effects of caffeine on attention tasks in healthy adult volunteers. Caffeine improves performance on simple and complex attention tasks, and affects the alerting, and executive control networks. Furthermore, there is inconclusive evidence on dose-related performance effects of caffeine, or the influence of habitual caffeine consumption on the performance effects of caffeine. Finally, caffeine's effects cannot be attributed to withdrawal reversal. Evidence shows that caffeine has clear beneficial effects on attention, and that these effects are even more widespread than previously assumed. 


\section{INTRODUCTION}

Caffeine is the single most widely consumed psychoactive ingredient (Ferre, 2008). Eighty percent of the world's population consumes caffeinated products every day, with coffee and tea being the primary caffeine sources (Heckman, Weil, \& Gonzalez de, 2010). Other natural dietary sources of caffeine are cacao beans, kola nuts, and guarana berries. Caffeine's popularity is attributed to its perceivable and acute benefits for physiological, psychomotor and cognitive performance, as well as its beneficial effects on mood. With regard to cognitive performance, the domain of attention appears to be most notably improved by caffeine consumption.

Although a number of reviews were conducted on the effects of caffeine on human behaviour and performance, (Lorist \& Tops, 2003; Rogers, 2007; Nehlig, 2010; Ruxton, 2008; Smith, 2002) an unequivocal conclusion about the specific effects of caffeine had not been reached. This could have been the result of the considerable variation within studies, in terms of methodology, types of tests used, caffeine dose consumed, and various sample characteristics such as age, gender, habitual caffeine use (Smith, 2002). Therefore, in the current review we endeavour to disentangle a number of these factors. Furthermore, while the scope of these reviews varied from effects of caffeine on performance in general, and cognition, to withdrawal and fatigue, they are by no means complete in representing the full range of literature at the time. Finally, in the past years since, a sizeable number of studies have been conducted on caffeine's attention performance effects. This review will serve as an update to existing reviews, by including a comprehensive literature search of the field of caffeine consumption and attention and including recent papers.

The aim of the current review is to determine the acute effects of caffeine on attention as measured in terms of performance on behavioural tasks. Furthermore, we wanted to investigate a number of very specific hypotheses about the effects of caffeine on attention, motivated by the fact that attention and psychomotor performance have been most extensively investigated in caffeine studies to date. Neuroimaging data such as ERP's, although related to behaviour, are not reviewed. Because of the complexity of the data, i.e. a multitude of different study designs, attention tests and test outcomes, a 
narrative review was preferred over a meta-analytical review. Specifically, a number of studies are multi-dose, multi-outcome, and multi-task studies, sometimes including up to five different tasks, and results can therefore not be considered as truly independent, thereby leading to underestimations of study-to-study variability. Hence, a narrative review better allowed capturing the richness of the data.

\section{Caffeine mechanism}

After oral consumption, caffeine is quickly and almost completely absorbed from the gastrointestinal tract and reaches peak plasma levels at about 30 minutes post consumption. Its elimination half-live ranges from 2.5 to 10 hours (Magkos \& Sidossis, 2005). Caffeine is rapidly distributed throughout the body and brain, where it exerts its effects (Blanchard \& Sawers, 1983; Dager \& Friedman, 2000). It does not accumulate in the body but it is extensively metabolized in the liver to more than 25 derivatives, while a small fraction (less than $5 \%$ of the ingested dose) is excreted unchanged in the urine (Magkos \& Sidossis, 2005).

It is well-established that caffeine's effects primarily stem from its ability to act as an adenosine antagonist (Ferre, 2008). Adenosine is a central nervous-system modulator. As it binds to its specific receptors in the brain, primarily the A1 and A2a receptors, adenosine slows down neural activity. As such the adenosine receptor system is thought to play an important role in sleep-wake regulation. Typically, adenosine levels accumulate over the course of the day only to decrease during sleep, and this is thought to increase the susceptibility to sleep after prolonged wakefulness (Porkka-Heiskanen, 1999).

As an adenosine antagonist, caffeine attenuates these inhibitory effects by interfering with adenosine receptors, resulting in caffeine's general wake-promoting effects but also its performance benefits. The pattern of distribution of A1 and A2a receptors in the brain differs strikingly, which explains the variety of effects that caffeine exerts (Daly, Jacobson, \& Ukena, 1987; Fisone, Borgkvist, \& Usiello, 2004; Smith, 2005). A1 receptors are widely distributed throughout the brain, with particularly high levels in the hippocampus, cerebral cortex and cerebellum, and in numerous hypothalamic nuclei. They inhibit the release of various neurotransmitters, such as glutamate, dopamine and acetylcholine. By binding to A1 receptors, caffeine blocks this inhibition, thereby in- 
creasing neuronal activity. This results in increased arousal, vigilance and attention. In contrast, A2a receptors are concentrated in the striatum and have been found to inhibit psychomotor function. Caffeine competes with adenosine at the A2 receptors and blocks this inhibition, which explains caffeine's ability to enhance psychomotor activity (Ferre, 2010). Caffeine also mediates vasoconstriction through action on A2 receptors. Hence, caffeine can have both neural and vascular effects depending on the ratio of A1 to A2 receptors in a given brain region (Pelegrino, $\mathrm{Xu}, \&$ Vetri, 2010).

By antagonizing adenosine, caffeine also affects the dopaminergic system; either by facilitating pre-synaptic dopamine release, or by stimulating dopaminergic neurotransmission via interactions between adenosine and dopamine receptors (Ferre, Fredholm, Morelli, Popoli, \& Fuxe, 1997). Neuroimaging studies indicate involvement of the brain's dopaminergic regions in arousal as well as higher order complex attention processes (Fan, McCandliss, Fossella, Flombaum, \& Posner, 2005). Therefore, it is likely that dopaminergic processes are affected by caffeine. Finally, caffeine also indirectly affects the release of various other neurotransmitters, including noradrenalin, acetylcholine, serotonin, glutamate and GABA (Nehlig, Daval, \& Debry, 1992). These neurotransmitters are widely distributed throughout the brain and are hypothesized to pertain to caffeine's general arousing effect.

\section{Attention processes}

Attention is an essential aspect of cognitive functioning and an important prerequisite for many cognitive processes, such as memory and reasoning. It allows the brains to effectively deal with the vast amount of input that is continuously received through its sensory (e.g. vision, hearing) and cognitive processes (e.g. memory). It is generally accepted that the concept of attention entails a number of distinct but related processes, but the exact structure of attention remains a matter of scientific debate (Raz \& Buhle, 2006). Throughout the last decades, various taxonomies of attention processes have been developed within different areas of research. However, within the context of the present review, we will focus on two of these taxonomies, the traditional division between simple and complex attention processes, and the Attention Network Theory (Posner \& Rothbart, 2007; Posner \& Petersen, 1990) for reasons outlined in the section below. 
The division into simple (bottom-up), basic attention processes and complex (top-down), real world attention processes (Jasso \& Triesch, 2007; Lim \& Dinges, 2010; Watson et al., 1988) is often referred to in reviews of attention effects of caffeine (Lorist \& Tops, 2003; Smith, 2002) but also in other related research areas, e.g. on sleep deprivation (Lim \& Dinges, 2010; Smith, 2002). Simple information processing is largely dependent on the relatively straightforward interactions between sensory input systems (i.e. a stimulus) and output systems (i.e. a response). These processes are bottom-up and rather automatic; an event actions a response (Lim \& Dinges, 2010). Tests generally involve the rapid detection of a single type of stimulus, without involvement of any form of executive attention. Simple information-processing tasks are sensitive to the arousal or psychomotor components of pharmacological interventions. On the contrary, higher order or complex attention tasks involve more demanding attention processes involving executive control, such as active coordination of behaviours required in processes such as inhibition of responses, decision making, and planning. It involves a greater degree of top-down processing (Lim \& Dinges, 2010). These processes are generally thought to be dependent on the prefrontal cortex (Miller \& Cohen, 2001). While previous reviews leave little doubt about the effects of caffeine on simple attention processing, effects on more complex aspects of attention processing are less wellestablished. Reviewing this specific distinction in our current review allows us to clearly build on previous reviews on caffeine and attention.

The second taxonomy employed here is the highly influential Attention Network Theory (Posner \& Rothbart, 2007; Posner \& Petersen, 1990), with nearly 3500 citations of the original publication, and which has been consistently supported by findings from brain imaging (Fan et al., 2005), behavioural (Fan, McCandliss, Sommer, Raz, \& Posner, 2002), genetic (Fan, Fossella, Sommer, Wu, \& Posner, 2003) and caffeine studies (Brunye, Mahoney, Lieberman, Giles, \& Taylor, 2010a; Brunye, Mahoney, Lieberman, Giles, \& Taylor, 2010b), Posner distinguishes between three functionally and anatomically different domains of attention, namely alerting, orienting, and executive control (Posner \& Rothbart, 2007; Posner \& Petersen, 1990). The strength of Posner's network theory is the anatomical base of the model: each of the three networks represents a different attention process, each with its own key brain regions and pharmacology. Alerting pertains to achieving and maintaining a state of high sensitivity to 
incoming stimuli, and involves dense dopaminergic regions, such as the thalamus and bilateral frontal and parietal brain regions (Fan et al., 2005). Orienting on the other hand, pertains to the ability to selectively attend to regions of space by directing attention to cued areas, and it involves the cholinergic system in the parietal lobe. Finally, executive control refers to the mechanisms for resolving conflict among thoughts, feelings, and responses. It involves the anterior cingulate cortex (ACC) and lateral prefrontal cortices (Botvinick, Braver, Barch, Carter, \& Cohen, 2001; Fan et al., 2005; Bush, Luu, \& Posner, 2000). As caffeine's pharmacology is well studied, the Posner taxonomy allows for clear hypotheses on caffeine's effects on the three networks.

\section{Hypotheses}

There is considerable variation within the vast literature of caffeine studies, in terms of methodology, the types of tests used, the caffeine dose consumed, and various sample characteristics (age, gender, habitual caffeine use). In the current review we seek to disentangle a number of these variables. The specific focus of this review will be restricted to the hypotheses mentioned below.

\section{Simple and complex attention processes}

Whereas there is a general consensus that caffeine can improve more basic aspects of attention (e.g. simple reaction time) via its role in increasing arousal, the evidence for its effects on more complex attention tasks is equivocal. Smith (2002) concluded that there was very limited evidence for beneficial effects of caffeine on more complex attention processes. This is somewhat surprising, as neuroimaging studies indicate the involvement of the brain's dopaminergic regions in higher order complex attention processes (Fan et al., 2005). Thus, these processes are likely to be affected by caffeine via its effect on dopamine. Since the review by Smith (2002), a number of studies have investigated the effect of caffeine on attention tasks that involve complex processes, such as task switching (Fan et al., 2005; Tieges, Snel, Kok, Wijnen, Lorist et al., 2006). Therefore, we will revisit this conclusion in the present review and hypothesize that the acute effects of caffeine consumption affect both simple and complex attention tasks (Hypothesis 1a). 
It has been suggested that effects of caffeine on complex attention are in fact secondary to or mediated by improvements in arousal and information processing (Nehlig, 2010). Caffeine results in improved processing of stimuli and preparation of motor responses, and may improve both complex and simple task performance via this mechanism. A number of recent studies contradict this view by showing that caffeine specifically enhanced complex attention processes. In fact, whereas caffeine consumption improved performance on the executive function component of the Attention Network Task, it did not significantly improve the more basic alerting component of this task (Brunye et al., 2010a; Brunye et al., 2010b; Giles, Mahoney, Brunye, Gardony, Taylor et al., 2012). Furthermore, an fMRI study showed that caffeine selectively modulated the BOLD signal in the frontal cortex and the right anterior cingulate, regions that are associated with executive functions such as motivated attention, error detection, planning and problem solving (Koppelstaetter, Poeppel, Siedentopf, Ischebeck, Kolbitsch et al., 2010). Hence, we hypothesize that the acute effect of caffeine consumption is not solely dependent on the effect it has on simple attention processes (Hypothesis $1 b)$.

Hypothesis 1a: $\quad$ Caffeine consumption affects both simple and complex attention processes.

Hypothesis 1b: $\quad$ The effect of caffeine consumption on complex attention processes is not solely dependent on its effects on simple attention processes.

\section{Attention networks}

Caffeine is known to strongly interact with the central dopaminergic systems. Areas related to alerting and executive function are high in dopamine receptors (Ferre, Fuxe, von, Johansson, \& Fredholm, 1992; Ferre, 2008; Lumme, Aalto, Ilonen, Nagren, \& Hietala, 2007). Thus, these domains are most likely to be affected by caffeine consumption. Brain areas involved in orienting on the other hand, show limited dopaminergic activity. On the basis of its dopaminergic stimulation it is unlikely for caffeine to affect the orienting network (Corbetta, Kincade, Ollinger, McAvoy, \& Shulman, 2000; Fan et 
al., 2005; Ferre, 2008). Therefore, it is hypothesised that the acute effects of caffeine consumption involve alerting and to some extent executive function, but not orienting.

Hypothesis 2: $\quad$ Caffeine consumption affects alerting and to some extent executive function, but not orienting.

\section{Caffeine dose levels}

Caffeine doses applied vary greatly, from as low as $12.5 \mathrm{mg}$ which is one-sixth of the amount of caffeine in a regular cup of coffee, up to as high as $500 \mathrm{mg}$ caffeine, the equivalent of 6 cups of coffee consumed at once. Whereas some studies have found effects of low doses of caffeine (12.5 mg: Smit \& Rogers, 2000; $40 \mathrm{mg}$ : Smith, Sturgess, \& Gallagher, 1999a), others have failed to find such effects. Furthermore, although some studies have found more pronounced effects of a high dose of caffeine (e.g. 400 mg: Brunye et al., 2010b), others indicate that effects of caffeine on performance seem to asymptote around $200 \mathrm{mg}$ (Fan et al., 2005; Brunye et al., 2010a; Robelin \& Rogers, 1998). In the present review, we will investigate whether effects of caffeine are dose-proportional.

Hypothesis 3: $\quad$ A higher caffeine dose exerts stronger attention effects.

\section{Habitual caffeine consumption}

Furthermore, it seems that the effects of these different caffeine doses on performance are difficult to interpret without taking habitual caffeine consumption of the participants into consideration. Notably, it has been shown that habitual consumption of caffeine can increase the number of adenosine receptors in the brain and thus high habitual caffeine consumers may require a greater caffeine dose to experience the same benefits of caffeine as a low habitual consumer (Daval, Von Lubitz, Deckert, \& Marangos, 1989; Fastbom, Post, \& Fredholm, 1990; Varani, Portaluppi, Merighi, Ongini, Belardinelli et al., 1999).

Hypothesis 4: Habitual caffeine consumers require a greater caffeine dose to experience attention effects. 


\section{Caffeine withdrawal}

Finally, an ongoing debate in the caffeine literature pertains to the influence of caffeine withdrawal. In the majority of studies on caffeine effects, volunteers were deprived of their habitual caffeine intake for a certain period preceding the intervention. Therefore, one may argue that caffeine's beneficial effects merely reflect the reversal of caffeine withdrawal (James \& Rogers, 2005; Keane \& James, 2008; Rogers, Heatherley, Hayward, Seers, Hill et al., 2005). With strong advocates on both sides, the topic of caffeine withdrawal remains controversial to date.

Hypothesis 5: $\quad$ The effect of acute caffeine consumption on attention is not influenced by withdrawal. 


\section{METHODS}

\section{Literature search}

In October 2010, the databases MEDLINE and PsychINFO were searched for relevant peer-reviewed journal articles, written in English. Searches terms included 'caffeine' in combination with 'attention', 'cognition', and 'cognitive function'. In line with the approach taken by Smith (2002), the selection was limited to papers published from 1992 onwards, as an extensive review covered the literature before 1992 (Lieberman, 1992) which mainly assessed more simple attention performance. As a next step, the reference lists of all pertinent papers were searched by hand to locate other relevant studies. Additional searches were conducted for the specific attention tasks that were encountered in the pertinent papers, in combination with the search term 'caffeine'. Finally, individual relevant articles on the acute effects of caffeine that appeared until July 2012 were also added to the review.

\section{Selection criteria}

Studies were included if they were double blind placebo controlled intervention studies (both parallel and crossover) that assessed the acute effects of at least one dose of caffeine on behavioural measures of attention in healthy adult volunteers $(\mathrm{N} \geq 10)$, and presented statistics directly relevant to the relation between caffeine and attention task performance. Studies using combinations of caffeine and other substances (e.g. glucose, herbs and drugs), or caffeine as part of a product consumed were excluded unless the results for caffeine as compared to a placebo could be extracted. Studies on long-term caffeine consumption were excluded, as well as studies in which volunteers were sleep deprived or otherwise challenged (except for caffeine deprivation). In total, 66 articles met these criteria. 


\section{Data extraction}

The following information was extracted from each paper and scored when required: (a) parallel or cross-over design, (b) duration of caffeine abstinence before intervention, (c) habitual daily caffeine, (d) caffeine intervention details (caffeine dose as reported in article, frequency of caffeine intervention), (e) attention task, (f) attention task outcome (reaction times, accuracy, etc.), (g) results per outcome.

\section{Test classification}

Test were classified according to the two divisions of attention domains (1. simple vs complex; 2. alerting, orienting, and executive control). Two independent researchers rated each task on the basis of the task description and the definitions of the attention domains. In cases of disagreement between the two, a third rater was asked to classify these particular tests and the classification with the majority of raters behind it was chosen. 


\section{RESULTS}

In 66 pertinent publications, 24 different tests of attention were employed, resulting in a total of 303 attention related outcomes. Table 1 summarizes all included studies, and study details. Table 2 presents the attention tests used and their categorization in terms of simple and complex, as well the attention network(s) they tap into. In the next sections, we will investigate our hypotheses.

\section{Hypothesis 1a: Caffeine consumption affects both simple and complex atten- tion processes.}

Using the predetermined criteria, 12 tests were identified as simple tests of attention and 12 as complex tests (see Table 2). Some tests have been used in a single study only, whereas others where more commonly used (e.g., Simple Reaction Time task).

\section{Simple attention processes}

Almost all tests demonstrate significant effects of various doses of caffeine on attention performance. Three tests that have been extensively used, namely the Repeat Digit Detection, Simple Reaction Time and a focused attention Choice Reaction Time test, shown consistent effects of a range of doses.

The Simple Reaction Time (SRT) test measures how fast people respond to the presence of a target stimulus. Additionally, few studies assessed the accuracy of responses in terms of correct hits (respond to target stimuli) (Childs \& de, 2006; Fine, Kobrick, Lieberman, Marlowe, Riley et al., 1994; Kelemen \& Creeley, 2001), false alarms (respond to non-target stimuli), or misses (failure to respond to target stimuli) (Martin \& Garfield, 2006). Caffeine (12.5 to $400 \mathrm{mg}$ ) significantly improved simple response speed in the vast majority of studies (see Table 2) and improved accuracy at the same time in some studies (Childs \& de, 2006; Fine et al., 1994; Kelemen \& Creeley, 2001). However, many studies did not report accuracy in relation to the SRT, most likely due to the fact that accuracy rates tend to be extremely high on the SRT. 
On the Repeat Digit Detection (RDD) test, in which subject are required to respond only to a specific three digit number, caffeine in a wide range of doses improved performance in 13 out of 15 studies. Specifically, caffeine improved response speed (Hewlett \& Smith, 2007; Christopher, Sutherland, \& Smith, 2005; Smith, Brice, Nash, Rich, \& Nutt, 2003), accuracy (Brice \& Smith, 2001; Hewlett \& Smith, 2007; Smith, Sutherland, \& Christopher, 2005) or both (Maridakis, O'Connor, \& Tomporowski, 2009; Maridakis, Herring, \& O'Connor, 2009; Smith, Kendrick, Maben, \& Salmon, 1994; Smith, 2009a; Smith, Kendrick, \& Maben, 1992).

Finally, a focused attention Choice Reaction Time (CRT) test, where participants were instructed to respond to the target letter presented in the centre and ignore peripheral distracters, also showed significant effects of caffeine; with caffeine mainly improving response speed (Christopher et al., 2005; Hewlett \& Smith, 2007; Hewlett \& Smith, 2006; Smith et al., 1999a; Smith et al., 2003; Smith, 2009a; Smith, Christopher, \& Sutherland, 2006) and sometimes simultaneously improved accuracy (Heatherley, Hayward, Seers, \& Rogers, 2005; Smith et al., 2005). Interestingly, it seemed that more consistent effects on the focused attention CRT were found after consumption of medium doses of caffeine (i.e. 80 to $150 \mathrm{mg}$ ).

With regard to the other simple attention tests, results were less consistent. A large variety of Choice Reaction Time tasks was used. Studies found faster responses on a 2-choice CRT task (Childs \& de, 2008; Addicott \& Laurienti, 2009; Haskell, Kennedy, Wesnes, \& Scholey, 2005; Smith et al., 2005) and more correct responses on a 5-choice CRT task (Smith, 2009a; Smith, 2009b; Smith, Brockman, Flynn, Maben, \& Thomas, 1993). It did seem that higher doses of caffeine (200 to $280 \mathrm{mg}$ ) were more likely to generate an effect on a CRT. Furthermore, inconsistent effects were found for the Continuous Performance Task (CPT).

The remaining simple attention tests encountered were employed in either one or two studies, which limits the conclusions to be drawn. Nevertheless, most of these tasks show some beneficial effects of caffeine. For example, Haskell and colleagues (Haskell et al., 2005; Haskell, Kennedy, Milne, Wesnes, \& Scholey, 2008) found that $150 \mathrm{mg}$ caffeine improved response times on a Digit Vigilance task, and others found improved accuracy and speed on a Selection task after $210 \mathrm{mg}$ caffeine (Kenemans \& Lorist, 1995), improved TOVA performance after 200 mg caffeine (Hunt, Momjian, \& Wong, 
2011), and improved response speed on an Auditory Go-No Go task after $250 \mathrm{mg}$ caffeine (Barry, Johnstone, Clarke, Rushby, Brown et al., 2007). No effects of caffeine were found on a Sequential Reaction Time task (Adan \& Serra-Grabulosa, 2010).

In sum, caffeine consumption positively affects both reaction times and accuracy on a variety of simple tasks.

\section{Complex attention processing}

Concerning attention tests of complex information processing, the majority of studies did find significant effects of caffeine. A Spatial Cuing Task showed no effects of 105 and $210 \mathrm{mg}$ caffeine (Kenemans \& Verbaten, 1998). All other tests employed found more or less consistent effects. Two tests in particular have been extensively used, namely a categorical search CRT and the Rapid Visual Information Processing (RVIP) task.

Performance on the RVIP was improved after caffeine in 10 out of 12 studies, either in terms of response speed (Hasenfratz \& Battig, 1994; Rees, Allen, \& Lader, 1999) more accuracy (Haskell et al., 2008; Smit \& Rogers, 2000) or both (Haskell et al., 2005; Kelemen \& Creeley, 2001; Maridakis et al., 2009; Maridakis et al., 2009; Warburton, Bersellini, \& Sweeney, 2001; Yeomans, Ripley, Davies, Rusted, \& Rogers, 2002). Interestingly, it seems that medium to high doses of caffeine (150 to $450 \mathrm{mg}$ ) mainly generate faster responses, while lower doses of caffeine (50 to $150 \mathrm{mg}$ ) mainly generate more accurate responses.

Furthermore, studies found improvements after caffeine on a categorical search CRT, similar to the focussed attention CRT task except that is was more complex as target letters appeared on the left or right side of the centre (Smith et al., 2005; Christopher et al., 2005; Hewlett \& Smith, 2006; Smith, 2009a; Smith, Clark, \& Gallagher, 1999b; Smith et al., 2006). Results gave no indications of a relation between caffeine dose and specificity of effects.

Other tests were less extensively used but also showed more or less consistent effects of caffeine on attention, except for a concentration task, which showed no differences between caffeine and placebo (Ruijter, Lorist, Snel, \& de Ruiter, 2000c). Using a Flanker Task (both original and adapted versions were used) significant improvements on response speed were found in three out of four studies after consumption of medium 
high doses of caffeine (150 to 250 mg:Addicott \& Laurienti, 2009; Tieges, Snel, Kok, \& Richard, 2009; Kenemans, Wieleman, Zeegers, \& Verbaten, 1999). Furthermore, studies employing the Stroop Task found reduced interference after $250 \mathrm{mg}$ caffeine, but not after $400 \mathrm{mg}$ or $100 \mathrm{mg}$ (Hasenfratz \& Battig, 1992; Tieges et al., 2009; Kenemans et al., 1999; Schmitt, Hogervorst, Vuurman, Jolles, \& Riedel, 2003). These effects are a tentative indication that some caffeine effects asymptote at a medium caffeine level. Various caffeine doses $(50,210,350 \mathrm{mg})$ also consistently improved performance on the Switch task, both reducing response speed and by improving accuracy (Owen et al., 2008; Tieges, Snel, Kok, Plat, \& Ridderinkhof, 2007; Tieges et al., 2006), and the Attention Network Task (ANT; Brunye et al., 2010a; Brunye et al., 2010b; Giles et al., 2012), mainly its executive control component.

Less frequently used tasks were the Cued Spatial Attention task (Kelly, GomezRamirez, Montesi, \& Foxe, 2008), Inhibition task (Heatherley et al., 2005), Selection task, (Ruijter, de Ruiter, \& Snel, 2000a; Ruijter, de Ruiter, Snel, \& Lorist, 2000b), Stop task (Childs \& de, 2006; Tieges et al., 2009) and a Visual search task (Lorist, Snel, Kok, \& Mulder, 1994; Lorist, Snel, Kok, \& Mulder, 1996). Most of these have shown some effects of caffeine (in doses ranging from 60 to $400 \mathrm{mg}$ ) on performance. However, because of the limited use of these tasks, no firm conclusions can be drawn.

A number of studies included a battery of several attention tasks. Therefore, one may wonder whether a proportion of the significant findings in these studies were due to chance. Data of interest to this question is presented in Table 1. This table summarizes all studies, including information on which tasks were administered and whether they showed a significant effect (as indicated by an asterisk). As can be seen, many studies which reported significant findings, only administered a single attention task. Next, we focused on studies which had used more than three outcome measures (Haskell et al., 2005; Christopher et al., 2005; Haskell et al., 2008; Hewlett \& Smith, 2006; Hewlett \& Smith, 2007; Smith et al., 1999a; Smith et al., 2003; Smith et al., 2005; Smith, 2009a; Smith et al., 2006). Closer inspection of these studies showed that, with the exception of the Smith et al. (2003) study, a consistent effect of caffeine is found on two or more tasks within each of these studies. Therefore, it is highly unlikely that significant findings of caffeine on attention tasks, which were administered in a battery of other attention tasks, are predominantly due to chance. 
To conclude on hypothesis 1a: not only did caffeine positively affect simple task performance, it also enhanced higher-order processes involved in the active monitoring and coordination of behaviour, such as executive control, task switching, inhibition, and interference.

\section{Hypothesis 1b: The effect of caffeine consumption on complex attention pro- cesses is not solely dependent on its effects on simple attention processes.}

It could be argued that effects of caffeine on complex attention are solely due to improvements in arousal and information processing. Fortunately, a number of the studies in this review ( $k=23$; Table 3$)$ have included both simple and complex attention tasks. Hence, investigating these particular studies can provide valuable insights into whether these effects tend to co-occur, thus supporting mediation, or whether complex processes are affected independent of simple processes.

Although a number of studies found effects on simple tasks only, two studies identified effects on specific complex tasks, but not on simple tasks: improved executive control in the absence of alerting effects on the ANT (Brunye et al., 2010b) improved switch task, but not CFF (Owen et al., 2008). The majority of studies however ( $k$ $=13$ ) found effects on simple as well as complex attention tasks within the same experiment. In three of these studies caffeine improved performance on some simple tasks but not others, while it did improve complex task performance. Only one study (Deslandes, Veiga, Cagy, Piedade, Pompeu et al., 2005) found no effects of caffeine on either task type.

Another approach to shed light on this matter is to compare pattern of results for response speed to accuracy findings. Specifically, one may hypothesize that improvements in arousal and information processing might have a much more pronounced effect on response speed, but only a minor effect on accuracy during complex tasks. As can be seen from Table 2, a number of studies using complex attention tasks reported significant findings of caffeine on accuracy only (Selection task) or response speed as well as accuracy (RVIP, Switch task). Hence, it seems that it cannot be concluded that mere improvements in speed underlie the effects of caffeine on complex processing. 
Some authors have specifically addressed this issue in their experimental design, and/or used imaging techniques such as event-related potentials (ERP) or fMRI to generate understanding on the specificity of the effect of caffeine on attention. Kenemans and Verbaten (1998) addressed the specificity of caffeine's effects using a Flanker task with various conditions, i.e. cued locations, target among distracters, and target among similar targets. Caffeine resulted in faster responses, but irrespective of the task conditions, and unparalleled by improvements in accuracy. Similarly, Ruijter and colleagues (2000b) concluded that caffeine does not have a specific influence on spatial attention per se, as signal detection parameters revealed that caffeine increased perceptual sensitivity as compared to placebo. Also, ERPs could not confirm that caffeine affected specific spatial attention components. Thus, these two studies seem to support the notion that caffeine exerts its effects on complex attention processes through its effect on simple attention processes. However, others do report improvements on specific processes are not merely due to increased processing and arousal, such as action monitoring (Tieges, Richard, Snel, \& Kok, 2004; Heckman et al., 2010) and anticipatory processes in task switching (Tieges et al., 2006).

To conclude on hypothesis $1 \mathrm{~b}$, although it seems likely that caffeine can affect separate brain processes resulting in improvements in both simple and complex attention tasks, further research is required to fully understand this relation and the specificity of the effects.

\section{Hypothesis 2: Caffeine consumption affects alerting and to some extent executive function, but not orienting.}

Only two studies employed the ANT, which specifically assesses all three networks, and found that alerting and executive control were most affected by caffeine (Brunye et al., 2010; Brunye et al., 2010b). With regard to the other studies, we would like to note that tasks can include various components of the Posner model, i.e. both an alerting component as well as an orienting component. Raters identified 13 tests containing an alerting component, five tests containing an orienting component, and 10 tests containing an executive control component (see Table 2). 


\section{Alerting}

The tests defined as containing an alerting component almost completely overlap with the tests defined as simple attention task in the previous section. Most of the tests have shown significant effects of various doses of caffeine on attention performance, and some of these have shown rather consistent improvements after caffeine consumption (Repeat Digit Detection, Simple Reaction Time, categorical search and focused attention Choice Reaction Time). In conclusion, and in line with the assumed mechanism of action for caffeine, consumption of various doses of caffeine can positively affect performance on an attention task which involves alerting.

\section{Orienting}

Five studies were classified as containing an orienting component. The categorical search CRT task demonstrated significant improvement following caffeine consumption. Three tasks were identified as having an orienting component namely the Spatial Cuing Task (Kenemans \& Verbaten, 1998), the Spatial Selection Task (Ruijter et al., 2000b) and a Visual Search Task (Lorist et al., 1994; Lorist et al., 1996). The tasks identified effects of caffeine on orienting, except for the Spatial Cuing Task, but were only employed once or twice each. The limited number of studies using an orienting component prevented drawing firm conclusions on the effect of caffeine on this specific attention component.

\section{Executive control}

Not surprisingly, as both involve prefrontal cortex activity, tasks including an executive control component overlapped with the tasks identified as complex tasks. Almost all studies found significant effects of caffeine on executive control (i.e. RVIP, Flanker Task, Stroop Task, Inhibition task, Visual Search Task, Stop Task, and Cued Spatial Attention Task). Most of these tasks have shown some effects of caffeine (in doses ranging from 60 to $400 \mathrm{mg}$ ) on attention performance. 
To conclude, caffeine positively affected performance on attention tasks which involve alerting, and executive control. The limited number of studies using an orienting component prevented firm drawing conclusions on the effect of caffeine on this specific attention component.

\section{Hypothesis 3: A higher caffeine dose exerts stronger attention effects.}

As studies varied on more than just doses used, one cannot easily and directly compare between the different studies. Therefore, we specifically reviewed studies, which included multiple doses of caffeine in a cross-over design, ranging from as little as $12.5 \mathrm{mg}$ to a high dose of $500 \mathrm{mg}$, and compared these to a caffeine-free placebo (see Table 4).

Only few studies clearly showed effects of caffeine that were dose-related. Warburton (1995) found that $150 \mathrm{mg}$ caffeine was more effective than $75 \mathrm{mg}$ on an RVIP task, while $200 \mathrm{mg}$ was more effective than 100mg on an RVIP and CPT task (Maridakis et al., 2009a). Furthermore, Smith et al. (2005) found a linear dose response relationship for caffeine, albeit that the higher dose $(3 \mathrm{mg} / \mathrm{kg})$ was in fact two repeated doses of $1.5 \mathrm{mg} / \mathrm{kg}$. Furthermore, Brunye et al. (2010b) found that performance effects of caffeine on alerting and executive control were most pronounced at $400 \mathrm{mg}$, albeit in a group of highly habitual caffeine consumers. In support of Brunyé's finding, Tieges et al. (2004) also found tentative evidence for better performance on a switch task after 5 $\mathrm{mg} / \mathrm{kg}(\sim 350 \mathrm{mg})$ then after $3 \mathrm{mg} / \mathrm{kg}(\sim 210 \mathrm{mg})$, as compared to placebo.

However, in contrast to these finding, Brunye et al. (2010a) found significant positive effects of caffeine on alerting and executive control after high doses (200 and $400 \mathrm{mg}$ ) rather than after $100 \mathrm{mg}$, but effects seemed to asymptote at $200 \mathrm{mg}$. With regard to the orienting network, they actually found decreased performance after caffeine, which was most pronounced after $400 \mathrm{mg}$. Furthermore, in a study by Hasenfratz and Battig (1994) effects appeared also to be selectively dose-related. Significant effects of caffeine included increased processing rate, but only after $1.5 \mathrm{mg} / \mathrm{kg}(\sim 100 \mathrm{mg})$ and 3 $\mathrm{mg} / \mathrm{kg}(\sim 210 \mathrm{mg})$, and decreased reaction time, but only after $1.5 \mathrm{mg} / \mathrm{kg}$ and $6 \mathrm{mg} / \mathrm{kg}$ ( 460 mg). Also, a trend for decreased commission errors was found after $1.5 \mathrm{mg} / \mathrm{kg}$, while commission errors tended to increase after $6 \mathrm{mg} / \mathrm{kg}$. Haskell et al. (2005) found that effects were positively dose-related for some tasks (Digit Vigilance accuracy) but 
negatively for others (RVIP) for 75 and $150 \mathrm{mg}$ caffeine. Finally, Child and de Wit (2006) found that a dose of $50 \mathrm{mg}$ was least effective in improving performance, and while 150 and $450 \mathrm{mg}$ caffeine both increased the number of hits in a vigilance task, only $450 \mathrm{mg}$ caffeine could decrease reaction time.

Two studies also found indications of a negative dose-response. Richardson et al. (1995) found that $70 \mathrm{mg}$ caffeine improved simple reaction time performance, whereas $250 \mathrm{mg}$ did not. Moreover, other studies showed no indication of dose-related effects. Rogers and Dernoncourt (1998) and Yeomans et al. (2002) found no difference in attention performance on various types of attention tasks between 1 and $2 \mathrm{mg} / \mathrm{kg}$ caffeine. Smith et al. (Smith et al., 1993) and Kenemans and Verbaten (1998) found no difference between 1.5 and $3 \mathrm{mg} / \mathrm{kg}$ caffeine, and neither did Tieges and colleagues (2006; 2007) between 3 and $5 \mathrm{mg} / \mathrm{kg}$ on complex attention tasks. Finally, Smit and Rogers (2000) found no difference between doses ranging from $12.5 \mathrm{mg}$ to $100 \mathrm{mg}$.

To conclude, from the studies reviewed there was inconclusive evidence on dose-related effects of caffeine. If anything, effects were likely to be apparent even at relatively low doses, but potentially asymptoted at middle to high doses (e.g. $200 \mathrm{mg}$ ).

\section{Hypothesis 4: Habitual caffeine consumers require a greater caffeine dose to experience attention effects.}

Nine of the studies under review compared different types of habitual caffeine consumers based on self-reported consumption by participants. Habitual consumption in studies varied from low habitual consumers (i.e. under $100 \mathrm{mg} /$ day) to moderate consumers (i.e. 100 to $200 \mathrm{mg} /$ day) and habitual high consumers (i.e. more than $200 \mathrm{mg} /$ day), also sometimes including non-consumers (see Table 5).

Caffeine improved performance in non-consumers, as well as low, and high habitual consumers (Fine et al., 1994; Haskell et al., 2005; Hewlett \& Smith, 2007; Richardson, Rogers, Elliman, \& O'Dell, 1995) regardless of habitual caffeine intake in most studies. However, effects of caffeine on performance were influenced by the participants' habitual consumption on some tasks, i.e. more complex tasks such as a CRT task, but not others, i.e. simple tasks such as the SRT or Digit Vigilance (Hewlett \& Smith, 2006; Smith et al., 2006). Specifically, effects of caffeine on performance were largest for high habitual consumers on the CRT and Focussed Attention CRT (Hewlett 
\& Smith, 2006; Smith et al., 2006) and highest for non-consumers on the Categoric Search CRT (Smith et al., 2006).

Furthermore, other studies reported that high habitual caffeine intake positively affected caffeine's performance effects, as significant effects of caffeine occurred only for high habitual consumers on the RVIP (Smit \& Rogers, 2000) and both the SRT and CRT (Attwood, Higgs, \& Terry, 2007). In contrast to the latter studies, but in line with findings by Smith and colleagues (2006), significant effects of caffeine on the Test of Variables of Attention (TOVA) only occurred for low habitual consumers (Hunt et al., 2011). Finally, one study did not report significant effects of caffeine and no conclusions on the different consumers groups can be drawn (Kennedy \& Haskell, 2011).

To conclude, there did not seem to be a consistent influence of habitual caffeine consumption on the performance effects of an acute dose of caffeine.

\section{Hypothesis 5: The effect of acute caffeine consumption on attention is not influenced by withdrawal}

In most caffeine studies volunteers were withdrawn from caffeine for 1 to 30 hours (most commonly 12 hours) at the time of the intervention. Therefore, it has been argued that the positive effect of caffeine on attention might reflect a reversal of caffeine withdrawal rather than an actual beneficial effect of caffeine. Different types of studies can provide insight into this issue: firstly, studies investigating the acute effects of caffeine consumption on volunteers who are not withdrawn from caffeine $(\mathrm{k}=6$; see Table 6$)$; secondly, studies in which volunteers were withdrawn only for a very short period (i.e. 1 to 2 hours) ( $\mathrm{k}=4$; see Table 7); thirdly, studies in which various abstinence levels were compared ( $\mathrm{k}=3$; see Table 8); fourthly, studies that investigate effects of caffeine on performance for volunteers who normally do not consume caffeine, or only in very low amount ( $\mathrm{k}=10$; see Table 5); fifthly, studies investigating the effects of withdrawal by administrating repeated doses of caffeine, i.e. a caffeine pre-load, in order to establish if a second dose has additional performance effects $(\mathrm{k}=5$; see Table 9). Furthermore, a number of researchers compared test data to baseline data or practice data in order to investigate the likelihood of withdrawal reversal. 
In studies where volunteers were not withdrawn (Addicott \& Laurienti, 2009; Childs \& de, 2006; Childs \& de, 2008; Kennedy \& Haskell, 2011; Smith et al., 2005) all but one (Smith, Whitney, Thomas, Perry, \& Brockman, 1997) have shown effects of caffeine (50 to $450 \mathrm{mg}$ ) on attention performance. Specifically, caffeine improved performance in terms of speed on the SRT (Childs \& de, 2006; Childs \& de, 2008), CRT (Addicott \& Laurienti, 2009; Childs \& de, 2006; Childs \& de, 2008; Christopher et al., 2005), focussed attention CRT and Repeat Digit task (Christopher et al., 2005) categorical search CRT task (Smith et al., 2006) and the Flanker task (Addicott \& Laurienti, 2009). Thus, caffeine seems to improve performance on a range of simple and complex tasks in non-withdrawn subjects (Table 6). In other studies, volunteers were withdrawn for a very short period, i.e. up to 2 hours (see Table 7). Here caffeine, in doses ranging from 40 to $250 \mathrm{mg}$, acutely improved response speed on the SRT (Rees et al., 1999; Richardson et al., 1995), focussed attention and categoric search CRT (Smith et al., 1999b), Repeat Digit Detection (Smith et al., 1994). Thus, studies on non-withdrawn or briefly withdrawn volunteers support the view that the effect of caffeine on performance is a net effect, independent of withdrawal.

Others have compared volunteers' performance after caffeine consumption following difference levels of abstinence (Table 8). Unfortunately, results from these studies are ambiguous. Whereas, Richardson et al. (1995) found effects of caffeine (70 mg) independent of abstinence level (1 $1 \frac{1}{2}$ hours, 12 hours or 7 days) on SRT, others show indications that effects are dependent on abstinence. Specifically, Heatherly and colleagues (2005) found that the effects of caffeine administration on SRT, CRT and Inhibition Task performance were largest after 8 hours (and in some cases 6 hours) abstinence, as compared to $4 \mathrm{hr}$ abstinence. Addicott and Laurienti (2009) also found that caffeine effects on the CRT were only present after a 30 hour abstinence. However, effects on the Flanker task were unrelated to abstinence levels in this study.

Further evidence for a net effect of caffeine comes from light habitual and nonconsumers of caffeine (Table 5). In a substantial number of studies, caffeine consumption (in doses ranging from 50 to $400 \mathrm{mg}$ ) has been shown to improve attention performance on various simple and complex tasks for volunteers who normally do not consume caffeine (Haskell et al., 2005; Richardson et al., 1995; Hewlett \& Smith, 2006; Smith et al., 2006) or low habitual thus non-dependent caffeine consumers (Brunye et 
al., 2010a; Childs \& de, 2006; Childs \& de, 2008; Hewlett \& Smith, 2006; Hewlett \& Smith, 2007). Fewer studies have found no significant effects of a low dose of caffeine (75 mg) in low habitual consumers (Adan \& Serra-Grabulosa, 2010; Kennedy \& Haskell, 2011; Serra-Grabulosa, Adan, Falcon, \& Bargallo, 2010). Finally, only one study found significant effects of a range of caffeine doses, but only for high habitual consumers (Smit \& Rogers, 2000). The totality of this data however indicates that caffeine significantly affects performance, also for light habitual and non-consumers of caffeine. These consumers are not dependent on caffeine and therefore do not suffer from withdrawal. This further supports that there is a net effect of caffeine consumption.

Other studies have controlled caffeine intake after withdrawal using a preserving of caffeine, equal for all volunteers (Table 9). Warburton et al. (1995), Smith (2005) and Hewlett et al. (2007) found that a second dose of caffeine, consumed 60 to 90 minutes after a caffeine pre-serving, improved performance. In contrast, others found no added effect of a $2^{\text {nd }}$ (and $3^{\text {rd }}$ ) serving of caffeine; in fact in the latter study no effects of caffeine were found when volunteers had consumed a pre-serving 1 hour before testing (Yeomans et al., 2002; Robelin \& Rogers, 1998). Thus, regarding pre-servings of caffeine, conclusions or caffeine withdrawal reversal are also equivocal.

Finally, a number of studies have specifically compared baseline performance for regular consumers, withdrawn from caffeine overnight, to baseline scores of nonconsumers and found no differences between groups (Hewlett \& Smith, 2006; Hewlett \& Smith, 2007; Smith et al., 2006). In addition, Brunye et al. (2010; 2010b) compared the data of the practice day, during which no restrictions were in place and no caffeine was consumed, to the data of the placebo day of the actually intervention, during which subject were withdrawn. In both studies, there were no significant differences between the practice day and the placebo day on any of the attention domains.

To conclude, although in some studies there was no indication of a net effect of caffeine, the majority of studies showed that caffeine effects cannot be attributed to withdrawal reversal. We conclude that relief from caffeine withdrawal seems unlikely to account for the beneficial effects of caffeine on attention. 


\section{DISCUSSION}

This review was set out to answer a number of questions to better understand the effects of caffeine on attention performance. Specifically, the types of tasks used, the caffeine dose consumed, habitual caffeine use and withdrawal were reviewed.

It can be concluded that both simple and complex attention task were consistently affected by caffeine consumption. Caffeine affected both reaction times and accuracy on a variety of simple tasks, but also had the potential to enhance higher-order processes involved in the active monitoring and coordination of behaviour, such as task switching, response inhibition, and interference. This is in line with neuroimaging studies that show the involvement of the brain's dopaminergic regions in arousal, as well as higher order complex attention processes (Fan et al., 2005).

It has been suggested that effects of caffeine on complex attention are secondary to or mediated by improvements in arousal and information processing (Nehlig, 2010). However, based on recent findings (Brunye et al., 2010a; Brunye et al., 2010b; Giles et al., 2012; Koppelstaetter et al., 2010) we hypothesized that the acute effect of caffeine consumption might not solely dependent on simple attention processes. However, most studies found clear effects on both types of tasks and made no effort to disentangle them. Therefore, no firm conclusions can be drawn from the current data to either support or decline our hypothesis. It should be noted that differences in robustness of effects between different tests of cognitive performance could be due to differences in test sensitivity. It seems likely that relatively simple test may experience less within and between subject variability than higher order attention tests. Unfortunately, only very few studies have included information on standard deviations, rendering it difficult to substantiate these assumptions.

As expected, the tests defined as containing an alerting component overlapped with tests defined as simple attention task in the previous section, whereas tasks containing an executive control component largely overlapped with the complex attention tasks. It can be concluded that, in line with the mechanism of action for caffeine, consumption of various doses of caffeine positively affected performance on attention tasks which involve alerting, and/or executive control. The limited number of studies using an 
orienting component prevented drawing conclusions on the effect of caffeine on this specific attention component. Furthermore, on the basis of the CNS effects of caffeine (mainly dopaminergic stimulation), it is unlikely for caffeine to affect the orienting network (Corbetta et al., 2000; Fan et al., 2005). However, the currently reported studies indicated that there may be a potential for caffeine to also affect the orienting component. This should be investigated further in future studies.

Furthermore, although some studies seemed to shown specific dose related effects of caffeine, generally effects of caffeine were attributed to a wide range of doses from 60 up to $450 \mathrm{mg}$ ). Only few studies out of a total of 17 relevant studies clearly showed effects of caffeine that were dose-related. Thus, there was inconclusive evidence on dose-related effects of caffeine. Furthermore, there was no consistent influence of habitual caffeine consumption on the performance effects of an acute dose of caffeine. Finally, an extensive review of all studies, which could potentially provide evidence for the withdrawal reversal by caffeine, showed that caffeine effects likely cannot be attributed to withdrawal reversal.

\section{Directions for future research}

There was a considerable amount of variation within caffeine studies. Studies varied not only in terms of methodology, types of tests used, and caffeine dose consumed, but also in various sample characteristics (age, gender, habitual caffeine use). In the current review we have disentangled some of these variables. Consumption of various doses of caffeine was found to positively affect performance and in terms of types of tasks, there were clear effects on both simple and complex attention task. No firm conclusions can be drawn, however, with regard to our hypothesis that effects of caffeine on complex attention are not solely dependent on improvements in arousal and information processing. Experiments employing tasks that include both simple and complex attention components (for example the ANT or Flanker task) and which then correct performance on complex components for performance on simple ones, or which employ neuroimaging techniques to identify the brain processes involved, could provide valuable insights to disentangling these effects. 
Furthermore, it was concluded that tasks which involve alerting and executive control components were clearly affected by caffeine consumption. The limited number of studies using an orienting component prevented drawing conclusions on the effect of caffeine on this specific attention component but current findings are indicative of some effects on this component as well. This should be investigated further in future studies.

All studies employed laboratory tests and it remains to be seen how these effects compare to real-life measures of attention. Few studies have found everyday real-world attention benefits, such as improved simulated driving (Brice \& Smith, 2001), greater alertness over the working day and smaller slowing of reaction times (Smith, 2005b), enhanced capabilities in military combat (McLellan, Kamimori, Voss, Bell, Cole et al., 2005a; McLellan, Kamimori, Bell, Smith, Johnson et al., 2005b) and improved perceived alertness during a university lecture (Peeling \& Dawson, 2007). Furthermore, a review of caffeine consumption and performance of shift works showed that caffeine can reduce the number of errors and improve cognitive performance during shift work (Ker, Edwards, Felix, Blackhall, \& Roberts, 2010). A recent study further extended the effects of caffeine on attention to a proofreading language task (Giles et al., 2012). Caffeine improved error detection and repair as a function of improved attentional control.

Finally, there are difficulties in translating caffeine consumption from experimental evidence to real life consumption situations. For example, coffee and tea are commonly consumed with a meal (e.g. breakfast) or just after a meal (e.g. dinner). A number of studies have addressed interactions between caffeine consumption and meals. Smith et al. (1994a; 1994b; 1997) and Hewlett and Smith (2007) found no interaction between breakfast or meals and caffeine with regard to effects on attention performance. Furthermore, Maridakis et al. (2009b) found that although the combination of carbohydrate and caffeine improved performance on one task, it did not on another. Also, while caffeinated drinks are commonly consumed throughout the day, most of the research has examined acute effects of single doses. A number of studies have shown that effects of caffeine may or may not disappear, but can also be enhanced by additional doses. This area also warrants further investigation. 


\section{TABLES AND FIGURES}

Table 1 Overview of pertinent placebo-controlled studies identified

\begin{tabular}{|c|c|c|c|c|c|c|c|}
\hline Study & Sample & $\begin{array}{l}\text { PA/ } \\
\text { CO }\end{array}$ & $T^{1}$ & $A^{2}$ & $\begin{array}{l}\text { Habitual } \\
\text { caffeine } \\
\text { intake }^{3} \\
\end{array}$ & $\begin{array}{l}\text { Attention } \\
\text { Task }^{4}\end{array}$ & Timing $^{5}$ \\
\hline $\begin{array}{l}\text { Adan et al. } \\
2010\end{array}$ & $\begin{array}{l}\mathrm{N}=72(\mathrm{MF}) \\
\mathrm{M}=21.07 \pm 1.7 \mathrm{y}\end{array}$ & PA & 75 & 18 & $<100 \mathrm{mg} / \mathrm{d}$ & $\begin{array}{l}\text { SRT, CRT, } \\
\text { Sequential } \\
\text { RT }\end{array}$ & 30 \\
\hline $\begin{array}{l}\text { Addicott \& } \\
\text { Laurienti } \\
2009\end{array}$ & $\begin{array}{l}\mathrm{N}=17(\mathrm{MF}) \mathrm{MF} \\
\mathrm{M}=30 \pm 8\end{array}$ & $\mathrm{CO}$ & 250 & 0,30 & $200-600 \mathrm{mg} / \mathrm{d}$ & $\begin{array}{l}\text { CRT*, Flank- } \\
\text { er Task* }\end{array}$ & 105 \\
\hline $\begin{array}{l}\text { Attwood et al. } \\
2007\end{array}$ & $\begin{array}{l}\mathrm{N}=49(\mathrm{MF}) \\
\mathrm{M}=23.2 \mathrm{y}\end{array}$ & $\mathrm{CO}$ & 400 & 14 & $\begin{array}{l}\mathrm{M}=200 \mathrm{mg} / \mathrm{d} \\
\mathrm{M}>200 \mathrm{mg} / \mathrm{d}\end{array}$ & $\begin{array}{l}\text { CRT*, RVIP, } \\
\text { SRT }\end{array}$ & 30 \\
\hline $\begin{array}{l}\text { Barry et al. } \\
2007\end{array}$ & $\begin{array}{l}\mathrm{N}=24(\mathrm{MF}) \\
\mathrm{M}=22.9 \mathrm{y}\end{array}$ & $\mathrm{CO}$ & 250 & 4 & coffee 24 cups/d & $\begin{array}{l}\text { Auditory } \\
\text { Go/No Go* }\end{array}$ & 35 \\
\hline $\begin{array}{l}\text { Brice \& } \\
\text { Smith } 2001\end{array}$ & $\begin{array}{l}\mathrm{N}=24(\mathrm{MF}) \\
19-34 \mathrm{y}\end{array}$ & $\mathrm{CO}$ & $3 \mathrm{mg} / \mathrm{kg}$ & N.A. & $\mathrm{M}=183.9 \mathrm{mg} / \mathrm{d}$ & RDD* & 30 \\
\hline $\begin{array}{l}\text { Brunyé et al. } \\
2010 \mathrm{a}\end{array}$ & $\begin{array}{l}\mathrm{N}=36(\mathrm{MF}) \\
\mathrm{M}=19.08 \mathrm{y}\end{array}$ & $\mathrm{CO}$ & $\begin{array}{l}100,200, \\
400\end{array}$ & 12 & $\mathrm{M}=42 \mathrm{mg} / \mathrm{d}$ & ANT* & 30 \\
\hline $\begin{array}{l}\text { Brunyé et al. } \\
2010 \mathrm{~b}\end{array}$ & $\begin{array}{l}\mathrm{N}=36(\mathrm{MF}) \\
\mathrm{M}=20.11\end{array}$ & $\mathrm{CO}$ & $\begin{array}{l}100,200, \\
400\end{array}$ & 12 & $\mathrm{M}=592.3 \mathrm{mg} / \mathrm{d}$ & ANT* & 30 \\
\hline $\begin{array}{l}\text { Childs \& De } \\
\text { Wit } 2006\end{array}$ & $\begin{array}{l}\mathrm{N}=102(\mathrm{MF}) \\
\mathrm{M}=21.6 \pm 0.3\end{array}$ & $\mathrm{CO}$ & $\begin{array}{l}50,150 \\
450\end{array}$ & 0 & $\mathrm{M}=116.7 \mathrm{mg} / \mathrm{w}$ & $\begin{array}{l}\text { SRT*, Stop } \\
\text { Task }\end{array}$ & 40 \\
\hline $\begin{array}{l}\text { Childs \& De } \\
\text { Wit } 2008\end{array}$ & $\begin{array}{l}\mathrm{N}=35(\mathrm{MF}) \\
18-35 \mathrm{y}\end{array}$ & $\mathrm{CO}$ & 200 & 0 & $\mathrm{M}=46.8 \mathrm{mg} / \mathrm{d}$ & SRT*, CRT* & 30 \\
\hline $\begin{array}{l}\text { Christopher et } \\
\text { al. } 2005\end{array}$ & $\begin{array}{l}\mathrm{N}=68(\mathrm{MF}) \\
\mathrm{M}=41 \mathrm{y}\end{array}$ & PA & $2 \mathrm{mg} / \mathrm{kg}$ & 0 & N.A. & $\begin{array}{l}\text { CRT-FA*, } \\
\text { CRT-CS*, } \\
\text { RDD*, SRT }\end{array}$ & 30 \\
\hline $\begin{array}{l}\text { Deslandes et } \\
\text { al. } 2005\end{array}$ & $\begin{array}{l}N=15(\mathrm{MF}) \\
\mathrm{M}=26 \pm 5 \mathrm{y}\end{array}$ & $\mathrm{CO}$ & 400 & 10 & coffee 12 cups/d & Stroop, SRT & 30 \\
\hline $\begin{array}{l}\text { Fine et al. } \\
1994\end{array}$ & $\begin{array}{l}\mathrm{N}=23(\mathrm{M}) \\
19-28 \mathrm{y}\end{array}$ & $\mathrm{CO}$ & 200 & 10 & N.A. & SRT* & 30 \\
\hline $\begin{array}{l}\text { Giles et al. } \\
2012\end{array}$ & $\begin{array}{l}\mathrm{N}=48(\mathrm{MF}) \\
\mathrm{M}=20.1 \pm 1.85 \mathrm{y}\end{array}$ & $\mathrm{CO}$ & 200 & 24 & $\begin{array}{l}\mathrm{M}=527.6 \\
\pm 277.1 \mathrm{mg} / \mathrm{d}\end{array}$ & $\begin{array}{l}\text { ANT }^{*}, \mathrm{SRT}^{*}, \\
\text { CRT* }^{*}\end{array}$ & $\begin{array}{l}30(\mathrm{ANT}), 70 \\
(\mathrm{SRT}, \mathrm{CRT})\end{array}$ \\
\hline $\begin{array}{l}\text { Hasenfratz \& } \\
\text { Bättig } 1992\end{array}$ & $\begin{array}{l}\mathrm{N}=20(\mathrm{~F}) \\
\mathrm{M}=28.3 \mathrm{y}\end{array}$ & $\mathrm{PA}$ & 250 & 12 & $\begin{array}{l}\text { coffee } M=5.4 \\
\text { cups } / \mathrm{d}\end{array}$ & Stroop* & 35 \\
\hline $\begin{array}{l}\text { Hasenfratz et } \\
\text { al. } 1994\end{array}$ & $\begin{array}{l}\mathrm{N}=20(\mathrm{~F}) \\
\mathrm{M}=33.4 \pm 7 \mathrm{y}\end{array}$ & $\mathrm{CO}$ & $\begin{array}{l}1.5,3,6 \\
\mathrm{mg} / \mathrm{kg}\end{array}$ & 12 & $\begin{array}{l}\text { coffee } M=6.4 \\
\text { cups } / \mathrm{d}\end{array}$ & RVIP* & 40 \\
\hline $\begin{array}{l}\text { Haskell et al. } \\
2005\end{array}$ & $\begin{array}{l}\mathrm{N}=48(\mathrm{MF}) \\
\mathrm{M}=23.8 \mathrm{y}\end{array}$ & $\mathrm{CO}$ & 75,150 & 12 & $\mathrm{M}=217 \mathrm{mg} / \mathrm{d}$ & $\begin{array}{l}\text { SRT }^{*}, \mathrm{DV}^{*}, \\
\text { CRT, RVIP* }\end{array}$ & 30 \\
\hline $\begin{array}{l}\text { Haskell et al. } \\
2008\end{array}$ & $\begin{array}{l}\mathrm{N}=24(\mathrm{MF}) \\
\mathrm{M}=21.3 \pm 0.83 \mathrm{y}\end{array}$ & $\mathrm{CO}$ & 150 & 12 & N.A. & $\begin{array}{l}\text { SRT*, CRT, } \\
\text { RVIP, DV* }\end{array}$ & 30,90 \\
\hline $\begin{array}{l}\text { Heatherly et } \\
\text { al. } 2005\end{array}$ & $\begin{array}{l}\mathrm{N}=49 \mathrm{MF} \\
\mathrm{M} \approx 22 \mathrm{y}\end{array}$ & $\mathrm{CO}$ & $1.2 \mathrm{mg} / \mathrm{kg}$ & $\begin{array}{l}4,6 \\
8\end{array}$ & $\begin{array}{l}\text { tea } / \text { coffee }< \\
3 / d\end{array}$ & $\begin{array}{l}\text { CRT FA*, } \\
\text { SRT*, Inhibi- } \\
\text { tion Task* }\end{array}$ & 30 \\
\hline $\begin{array}{l}\text { Hewlett et al. } \\
2006\end{array}$ & $\begin{array}{l}\mathrm{N}=176 \\
\mathrm{M}=23.5 \pm 8 \mathrm{y}\end{array}$ & PA & $\begin{array}{l}1 \mathrm{mg} / \mathrm{kg} \\
(2 \mathrm{x})\end{array}$ & 7 & $\begin{array}{l}\mathrm{M}=\sim 200 \mathrm{mg} / \mathrm{d} \\
\mathrm{M}=\sim 50 \mathrm{mg} / \mathrm{d} \\
\text { non consumers }\end{array}$ & $\begin{array}{l}\text { CRT-FA, } \\
\text { CRT-CS*, } \\
\text { RDD*, SRT* }\end{array}$ & $\begin{array}{l}\text { t1 } 60,180 \\
\text { t2 240/ T1, } \\
60 / \mathrm{T} 2\end{array}$ \\
\hline $\begin{array}{l}\text { Hewlett et al. } \\
2007\end{array}$ & $\begin{array}{l}\mathrm{N}=120(\mathrm{MF}) \\
\mathrm{M} \approx 24 \mathrm{y}\end{array}$ & PA & $\begin{array}{l}1 \mathrm{mg} / \mathrm{kg} \\
(2 \mathrm{x})\end{array}$ & 7 & $\begin{array}{l}\mathrm{M}=212 \mathrm{mg} / \mathrm{d} \\
\mathrm{M}=53.6 \mathrm{mg} / \mathrm{d}, \\
\text { non consumers }\end{array}$ & $\begin{array}{l}\text { CRT-FA, } \\
\text { CRT-CS, } \\
\text { RDD*, SRT* }\end{array}$ & 60 \\
\hline $\begin{array}{l}\text { Hunt et al. } \\
2011\end{array}$ & $\begin{array}{l}\mathrm{N}=63(\mathrm{MF}) \\
\mathrm{M}=19 \mathrm{y}\end{array}$ & PA & 200 & $\begin{array}{l}15- \\
18 \\
\end{array}$ & $\begin{array}{l}\mathrm{M}=167 \pm 207 \\
\mathrm{mg} / \mathrm{d}\end{array}$ & TOVA* & 30 \\
\hline
\end{tabular}




\begin{tabular}{|c|c|c|c|c|c|c|c|}
\hline Study & Sample & $\begin{array}{l}\text { PA/ } \\
\text { CO }\end{array}$ & $T^{1}$ & $\mathbf{A}^{2}$ & $\begin{array}{l}\text { Habitual } \\
\text { caffeine } \\
\text { intake }^{3} \\
\end{array}$ & $\begin{array}{l}\text { Attention } \\
\text { Task }^{4}\end{array}$ & Timing $^{5}$ \\
\hline $\begin{array}{l}\text { Kelemen et } \\
\text { al. } 2001\end{array}$ & $\begin{array}{l}\mathrm{N}=142(\mathrm{MF}) \\
\mathrm{M} \approx 25 \mathrm{y}\end{array}$ & PA & $\begin{array}{l}4 \mathrm{mg} / \\
\mathrm{kg}\end{array}$ & 3 & $\mathrm{M} \approx 120 \mathrm{mg} / \mathrm{d}$ & SRT* & 30 \\
\hline $\begin{array}{l}\text { Kelly et al. } \\
2008\end{array}$ & $\begin{array}{l}\mathrm{N}=16(\mathrm{MF}) \\
\mathrm{M}=27.5 \mathrm{y}\end{array}$ & $\mathrm{CO}$ & 50 & 24 & $\begin{array}{l}\text { coffee } M=3.7 \\
\text { cups } / w \\
\text { tea } M=3.8 \\
\text { cups } / w\end{array}$ & $\begin{array}{l}\text { Spatial cuing } \\
\text { Task* }\end{array}$ & 30 \\
\hline $\begin{array}{l}\text { Kenemans \& } \\
\text { Lorist } 1995\end{array}$ & $\begin{array}{l}\mathrm{N}=16(\mathrm{MF}) \\
\mathrm{M}=21.5 \mathrm{y}\end{array}$ & $\mathrm{CO}$ & $3 \mathrm{mg} / \mathrm{kg}$ & 12 & $\begin{array}{l}\text { coffee } M=5.9 \\
\pm 2.6 \mathrm{cups} / \mathrm{d}\end{array}$ & $\begin{array}{l}\text { Selection } \\
\text { Task* }\end{array}$ & 45 \\
\hline $\begin{array}{l}\text { Kenemans \& } \\
\text { Verbaten } \\
1998 \\
\end{array}$ & $\begin{array}{l}\mathrm{N}=24 \\
\mathrm{M}=23.2 \mathrm{y}\end{array}$ & $\mathrm{CO}$ & $\begin{array}{l}1.5,3 \\
\mathrm{mg} / \mathrm{kg}\end{array}$ & 12 & $\begin{array}{l}\text { coffee } 4-8 \\
\text { cups } / \mathrm{d}\end{array}$ & $\begin{array}{l}\text { Spatial Cuing } \\
\text { Task, Flanker } \\
\text { Task* }\end{array}$ & 45 \\
\hline \multirow{2}{*}{$\begin{array}{l}\text { Kenemans et } \\
\text { al. 1999; } \\
\text { (study 1; } \\
\text { study 2) } \\
\end{array}$} & $\begin{array}{l}\mathrm{N}=16(\mathrm{~F}) \\
\mathrm{M}=23.8 \pm 2.8 \mathrm{y}\end{array}$ & $\mathrm{CO}$ & 250 & 12 & $\begin{array}{l}\text { coffee } \mathrm{M}=3.4 \\
\pm 1.5 \mathrm{cups} / \mathrm{d}\end{array}$ & Stroop* & 45 \\
\hline & $\begin{array}{l}\mathrm{N}=16(\mathrm{~F}) \\
\mathrm{M}=22.1 \pm 2.1 \mathrm{y}\end{array}$ & $\mathrm{CO}$ & 250 & 12 & $\begin{array}{l}\text { coffee } M=4 \pm \\
3.2 \text { cups/d }\end{array}$ & Stroop* & 45 \\
\hline $\begin{array}{l}\text { Kennedy \& } \\
\text { Haskell } 2011\end{array}$ & $\begin{array}{l}\mathrm{N}=20(\mathrm{MF}) \\
\mathrm{M}=21.4 \mathrm{y}\end{array}$ & $\mathrm{CO}$ & 75 & $\begin{array}{l}15- \\
18\end{array}$ & $\begin{array}{l}\mathrm{M}=667 \mathrm{mg} / \mathrm{d}, \\
\mathrm{M}=333 \mathrm{mg} / \mathrm{d}\end{array}$ & RVIP* & 30 \\
\hline $\begin{array}{l}\text { Lorist \& Snel } \\
1997\end{array}$ & $\begin{array}{l}\mathrm{N}=16(\mathrm{MF}) \\
\mathrm{M}=21.8 \pm 2.8 \mathrm{y}\end{array}$ & $\mathrm{CO}$ & $\begin{array}{l}3 \mathrm{mg} / \mathrm{kg} \\
\mathrm{mg}\end{array}$ & 12 & $\begin{array}{l}\text { coffee } M=4.5 \\
\pm 2.1 \mathrm{cups} / \mathrm{d}\end{array}$ & Flanker Task & 70 \\
\hline $\begin{array}{l}\text { Lorist et al. } \\
1994\end{array}$ & $\begin{array}{l}\mathrm{N}=30(\mathrm{MF}) \\
18-25 \mathrm{y}\end{array}$ & $\mathrm{CO}$ & $\begin{array}{l}200+ \\
50 \\
\end{array}$ & 12 & $\begin{array}{l}\text { coffee } M=27 \\
\text { cups } / \mathrm{d}\end{array}$ & $\begin{array}{l}\text { Visual search } \\
\text { Task* }\end{array}$ & n.a. \\
\hline $\begin{array}{l}\text { Lorist et al. } \\
1996\end{array}$ & $\begin{array}{l}\mathrm{N}=16(\mathrm{MF}) \\
\mathrm{M}=21.5 \pm 2.9 \mathrm{y}\end{array}$ & $\mathrm{CO}$ & $\begin{array}{l}3 \mathrm{mg} / \mathrm{kg} \\
\mathrm{mg}\end{array}$ & 12 & $\begin{array}{l}\text { coffee } M=5.9 \\
\pm 2.6 \mathrm{cups} / \mathrm{d}\end{array}$ & $\begin{array}{l}\text { Visual search } \\
\text { Task* }\end{array}$ & 45 \\
\hline $\begin{array}{l}\text { Mackay et al. } \\
2002\end{array}$ & $\begin{array}{l}\mathrm{N}=64(\mathrm{M}) \\
\mathrm{M}=21.3 \mathrm{y}\end{array}$ & PA & 120 & 12 & N.A. & CRT & 20 \\
\hline $\begin{array}{l}\text { Maridakis et } \\
\text { al. } 2009 a\end{array}$ & $\begin{array}{l}\mathrm{N}=18(\mathrm{MF}) \\
\mathrm{M}=22.7 \pm 4 \mathrm{y}\end{array}$ & $\mathrm{CO}$ & $\begin{array}{l}100 \\
200\end{array}$ & 8 & N.A. & $\begin{array}{l}\text { Bakan 1 } \\
(\sim \text { RVIP)*, } \\
\text { Bakan } 2 \\
(\sim \text { RDD)* }\end{array}$ & 20 \\
\hline $\begin{array}{l}\text { Maridakis et } \\
\text { al. } 2009 \mathrm{~b}\end{array}$ & $\begin{array}{l}\mathrm{N}=17(\mathrm{MF}) \\
\mathrm{M}=22.7 \pm 4 \mathrm{y}\end{array}$ & $\mathrm{CO}$ & 200 & 8 & N.A. & $\begin{array}{l}\text { Bakan 1*, } \\
\text { Bakan 2*, } \\
\text { CPT-AX* }\end{array}$ & 20 \\
\hline $\begin{array}{l}\text { Martin \& } \\
\text { Garfield } 2006\end{array}$ & $\begin{array}{l}\mathrm{N}=16(\mathrm{~F}) \\
\mathrm{M}=24 \pm 7 \mathrm{y}\end{array}$ & $\mathrm{CO}$ & $100 \mathrm{mg}$ & 12 & $\mathrm{M}=153 \mathrm{mg} / \mathrm{d}$ & SRT, CRT* & 45 \\
\hline $\begin{array}{l}\text { Owen et al. } \\
2008\end{array}$ & $\begin{array}{l}\mathrm{N}=27(\mathrm{MF}) \\
\mathrm{M}=28.3 \pm 5.3 \mathrm{y}\end{array}$ & $\mathrm{CO}$ & 50 & 12 & $\begin{array}{l}\text { coffee } M=9.6 \\
\text { cups } / w\end{array}$ & $\begin{array}{l}\text { RVIP*, } \\
\text { Switch } \\
\text { Task*, CFF }\end{array}$ & 60,90 \\
\hline $\begin{array}{l}\text { Rees et al. } \\
1999\end{array}$ & $\begin{array}{l}\mathrm{N}=48(\mathrm{MF}) \\
\mathrm{M}=23.5 / 56.5 \mathrm{y}\end{array}$ & PA & 250 & 2 & N.A. & $\begin{array}{l}\text { SRT*, CFF*, } \\
\text { RVIP* }\end{array}$ & 60 \\
\hline $\begin{array}{l}\text { Richardson et } \\
\text { al. } 1995\end{array}$ & $\begin{array}{l}\mathrm{N}=67(\mathrm{MF}) \\
18-23 \mathrm{y}\end{array}$ & $\mathrm{CO}$ & 70,250 & $\begin{array}{l}11 / 2 \\
12 \\
7 d \\
\end{array}$ & $\begin{array}{l}\mathrm{M}<15 \mathrm{mg} / \mathrm{d}, \mathrm{M} \\
=200-1000 \\
\mathrm{mg} / \mathrm{d}\end{array}$ & SRT* & 45 \\
\hline $\begin{array}{l}\text { Robelin \& } \\
\text { Rogers } 1998\end{array}$ & $\begin{array}{l}\mathrm{N}=64(\mathrm{MF}) \\
\mathrm{M}=33 \mathrm{y}\end{array}$ & PA & $1.2 \mathrm{mg} / \mathrm{kg}$ & 12 & $\mathrm{M}=465 \mathrm{mg} / \mathrm{d}$ & SRT* & $\begin{array}{l}\mathrm{t} 145 \\
\mathrm{t} 2120 / \mathrm{T} 1, \\
45 / \mathrm{T} 2 \\
\mathrm{t} 3210 / \mathrm{T} 1, \\
135 / \mathrm{T} 2,45 / \\
\mathrm{T} 3\end{array}$ \\
\hline $\begin{array}{l}\text { Rogers \& } \\
\text { Dernoncourt } \\
1998 \\
\end{array}$ & $\begin{array}{l}\mathrm{N}=18(\mathrm{MF}) \\
\mathrm{M}=26.7 \pm 0.7 \mathrm{y} \\
\mathrm{M}=62.6 \pm 1.1 \mathrm{y}\end{array}$ & $\mathrm{CO}$ & $\begin{array}{l}1,2 \\
\mathrm{mg} / \mathrm{kg}\end{array}$ & 14 & $\begin{array}{l}\mathrm{M}= \\
6.4 \mathrm{mg} / \mathrm{kg} / \mathrm{d} ; \mathrm{M} \\
=8.94 \mathrm{mg} / \mathrm{kg} / \mathrm{d}\end{array}$ & SRT* & 45 \\
\hline $\begin{array}{l}\text { Ruijter et al. } \\
2000 \mathrm{a}\end{array}$ & $\begin{array}{l}\mathrm{N}=11(\mathrm{MF}) \\
\mathrm{M}=22.4 \pm 2.0 \mathrm{y}\end{array}$ & $\mathrm{CO}$ & 250 & 12 & $\begin{array}{l}\text { coffee } M=4.9 \\
\text { cups } / \mathrm{d}\end{array}$ & $\begin{array}{l}\text { Selection } \\
\text { Task* }\end{array}$ & 40 \\
\hline $\begin{array}{l}\text { Ruijter et al. } \\
2000 \mathrm{~b}\end{array}$ & $\begin{array}{l}\mathrm{N}=11(\mathrm{MF}) \\
\mathrm{M}=22.4 \pm 2.0 \mathrm{y}\end{array}$ & $\mathrm{CO}$ & 250 & 12 & $\begin{array}{l}\text { coffee } M=4.9 \\
\text { cups/d }\end{array}$ & $\begin{array}{l}\text { Selection } \\
\text { Task* }\end{array}$ & 40 \\
\hline Ruijter et al. & $\mathrm{N}=12(\mathrm{MF})$ & $\mathrm{CO}$ & 250 & 12 & coffee $M=4.9$ & Concentration & 40 \\
\hline
\end{tabular}




\begin{tabular}{|c|c|c|c|c|c|c|c|}
\hline Study & Sample & $\begin{array}{l}\text { PA/ } \\
\text { CO }\end{array}$ & $T^{1}$ & $\mathbf{A}^{2}$ & $\begin{array}{l}\text { Habitual } \\
\text { caffeine } \\
\text { intake }^{3} \\
\end{array}$ & $\begin{array}{l}\text { Attention } \\
\text { Task }^{4}\end{array}$ & Timing $^{5}$ \\
\hline $2000 c$ & $\mathrm{M}=22.4 \pm 1.9 \mathrm{y}$ & & & & cups/d & Task & \\
\hline $\begin{array}{l}\text { Schmitt et al. } \\
2003\end{array}$ & $\begin{array}{l}\mathrm{N}=30(\mathrm{MF}) \\
\mathrm{M}=54.6 \pm 3.7 \mathrm{y} \\
\mathrm{M}=67.7 \pm 5.1 \mathrm{y}\end{array}$ & $\mathrm{CO}$ & 100 & 12 & N.A. & Stroop & 30 \\
\hline $\begin{array}{l}\text { Serra- } \\
\text { Grabulosa et } \\
\text { al. } 2010\end{array}$ & $\begin{array}{l}\mathrm{N}=40(\mathrm{MF}) \\
\mathrm{M}=19.6 \pm 1.7 \mathrm{y}\end{array}$ & $\mathrm{PA}$ & 75 & 12 & $\mathrm{M}<100 \mathrm{mg} / \mathrm{d}$ & CPT & 30 \\
\hline $\begin{array}{l}\text { Smit \& } \\
\text { Rogers } 2000\end{array}$ & $\begin{array}{l}\mathrm{N}=23(\mathrm{MF}) \\
\mathrm{M}=31.6 \pm 12.1 \mathrm{y}\end{array}$ & $\mathrm{CO}$ & $\begin{array}{l}12.5,25 \\
50,100\end{array}$ & 12 & $\begin{array}{l}\mathrm{M}<100 \mathrm{mg} / \mathrm{d} \\
\mathrm{M}>200 \mathrm{mg} / \mathrm{d}\end{array}$ & SRT*, RVIP* & $2,34,66$ \\
\hline $\begin{array}{l}\text { Smith et al. } \\
1992\end{array}$ & $\mathrm{~N}=48(\mathrm{MF})$ & $\mathrm{PA}$ & $4 \mathrm{mg} / \mathrm{kg}$ & N.A. & $\begin{array}{l}\text { coffee } M=2 \\
\text { cups } / \mathrm{d}\end{array}$ & RDD* & N.A. \\
\hline $\begin{array}{l}\text { Smith et al. } \\
1993\end{array}$ & $\mathrm{~N}=24(\mathrm{MF})$ & $\mathrm{CO}$ & $\begin{array}{l}1,5 \text { or } 3 \\
\mathrm{mg} / \mathrm{kg}\end{array}$ & N.A. & $\begin{array}{l}\text { coffee } M=24 \\
\text { cups } / d\end{array}$ & SRT $^{*}, \mathrm{CRT}^{*}$ & $\begin{array}{l}\mathrm{t} 130 \\
\mathrm{t} 2150 / \mathrm{T} 1, \\
30 / \mathrm{T} 2 \\
\mathrm{t} 3300 / \mathrm{T} 1, \\
150 / \mathrm{T} 2,60 / \\
\mathrm{T} 3 \\
\mathrm{t} 4420 / \mathrm{T} 1, \\
270 / \mathrm{T} 2,120 / \\
\mathrm{T} 3,30 / \mathrm{T} 4\end{array}$ \\
\hline $\begin{array}{l}\text { Smith et al. } \\
1994 \mathrm{a}\end{array}$ & $\mathrm{N}=48(\mathrm{MF})$ & $\mathrm{PA}$ & $4 \mathrm{mg} / \mathrm{kg}$ & 12 & N.A. & $\begin{array}{l}\text { SRT*, RDD*, } \\
\text { CRT }\end{array}$ & 30,90 \\
\hline $\begin{array}{l}\text { Smith et al. } \\
1994 b\end{array}$ & $\mathrm{~N}=48(\mathrm{MF})$ & PA & $3 \mathrm{mg} / \mathrm{kg}$ & 1 & N.A. & $\begin{array}{l}\text { SRT*, RDD*, } \\
\text { CRT }\end{array}$ & 60,150 \\
\hline $\begin{array}{l}\text { Smith et al. } \\
1997\end{array}$ & $\begin{array}{l}\mathrm{N}=106(\mathrm{MF}) \\
\mathrm{M}=21.2 \pm 3.4 \mathrm{y}\end{array}$ & $\mathrm{PA}$ & $\begin{array}{l}1.5 \\
\mathrm{mg} / \mathrm{kg}\end{array}$ & 0 & N.A. & $\begin{array}{l}\text { SRT, RDD, } \\
\text { CRT }\end{array}$ & 60 \\
\hline $\begin{array}{l}\text { Smith et al. } \\
1999 \text { a }\end{array}$ & $\begin{array}{l}\mathrm{N}=144 \\
\mathrm{M}=21 \mathrm{y}\end{array}$ & $\mathrm{PA}$ & 40 & 2 & N.A. & $\begin{array}{l}\text { CRT, CRT- } \\
\text { FA*, CRT- }^{*} \text { CS*, RDD, }^{*} \\
\text { CRT }\end{array}$ & 60 \\
\hline $\begin{array}{l}\text { Smith et al. } \\
1999 b\end{array}$ & $\begin{array}{l}\mathrm{N}=144(\mathrm{MF}) \\
\mathrm{M}=21 \mathrm{y}\end{array}$ & PA & 200 & 2 & N.A. & CRT-CS * & $\begin{array}{l}\text { t1 } 60 \\
\text { t2 120/T1, } \\
60 / \mathrm{T} 2 \\
\end{array}$ \\
\hline $\begin{array}{l}\text { Smith et al. } \\
2003\end{array}$ & $\begin{array}{l}\mathrm{N}=24(\mathrm{M}) \\
\mathrm{M}=22.6 \pm 0.8 \mathrm{y}\end{array}$ & PA & $\begin{array}{l}1.5 \\
\mathrm{mg} / \mathrm{kg}\end{array}$ & 8 & $\begin{array}{l}\mathrm{M}=178.1 \mathrm{mg} \\
/ \mathrm{d}\end{array}$ & $\begin{array}{l}\text { CRT, CRT- } \\
\text { FA, CRT-CS, } \\
\text { RDD*, SRT }\end{array}$ & $\begin{array}{l}\text { t1 }-30 \\
\text { t2 - 150, } \\
270 / \mathrm{T} 1,80, \\
\text { 200/T2 }\end{array}$ \\
\hline $\begin{array}{l}\text { Smith et al. } \\
2005\end{array}$ & $\begin{array}{l}\mathrm{N}=60(\mathrm{MF}) \\
\mathrm{M}=21 \mathrm{y}\end{array}$ & $\mathrm{CO}$ & $\begin{array}{l}1.5,1.5 \\
(2 \mathrm{x}) \\
\mathrm{mg} / \mathrm{kg}\end{array}$ & 0 & N.A. & $\begin{array}{l}\text { CRT-FA*, } \\
\text { CRT-CS*, } \\
\text { RDD*, SRT* }\end{array}$ & $\begin{array}{l}\mathrm{t} 1-30 \\
\mathrm{t} 2-90 / \mathrm{T} 1 \\
30 / \mathrm{T} 2\end{array}$ \\
\hline $\begin{array}{l}\text { Smith et al. } \\
2006\end{array}$ & $\begin{array}{l}\mathrm{N}=50(\mathrm{MF}) \\
\mathrm{M} \approx 21 \mathrm{y}\end{array}$ & $\mathrm{CO}$ & $2 \mathrm{mg} / \mathrm{kg}$ & 16 & N.A. & $\begin{array}{l}\text { CRT-FA*, } \\
\text { CRT-CS*, } \\
\text { RDD*, SRT* }\end{array}$ & 25 \\
\hline $\begin{array}{l}\text { Smith et al. } \\
2009\end{array}$ & $\begin{array}{l}\mathrm{N}=118(\mathrm{MF}) \\
\text { N.A. (students) }\end{array}$ & PA & 40 & 6 & $\mathrm{M}=138 \mathrm{mg} / \mathrm{d}$ & $\begin{array}{l}\text { CRT*, CRT- } \\
\text { FA, CRT-CS, } \\
\text { RDD*, SRT, }\end{array}$ & $20-80$ \\
\hline $\begin{array}{l}\text { Tieges et al. } \\
2004\end{array}$ & $\begin{array}{l}15(\mathrm{MF}) \\
\mathrm{M}=20.4 \pm 2.3 \mathrm{y}\end{array}$ & $\mathrm{CO}$ & $\begin{array}{l}3,5 \\
\mathrm{mg} / \mathrm{kg}\end{array}$ & 12 & $\begin{array}{l}\text { coffee } M=3.66 \\
\mathrm{p} / \mathrm{d} \pm 1.24\end{array}$ & Switch Task* & 45 \\
\hline $\begin{array}{l}\text { Tieges et al. } \\
2006\end{array}$ & $\begin{array}{l}\mathrm{N}=18(\mathrm{MF}) \\
\mathrm{M}=20.9 \pm 3.1 \mathrm{y}\end{array}$ & $\mathrm{CO}$ & $\begin{array}{l}3,5 \\
\mathrm{mg} / \mathrm{kg}\end{array}$ & 12 & $\begin{array}{l}\mathrm{M}=406 \\
\pm 135 \mathrm{mg} / \mathrm{d}\end{array}$ & Switch Task* & 45 \\
\hline $\begin{array}{l}\text { Tieges et al. } \\
2007\end{array}$ & $\begin{array}{l}\mathrm{N}=18(\mathrm{MF}) \\
\mathrm{M}=21.6 \pm 3.6 \mathrm{y}\end{array}$ & $\mathrm{CO}$ & $\begin{array}{l}3,5 \\
\mathrm{mg} / \mathrm{kg}\end{array}$ & 12 & $\begin{array}{l}M=44 \pm \\
136 \mathrm{mg} / \mathrm{d}\end{array}$ & Switch Task* & 45 \\
\hline $\begin{array}{l}\text { Tieges et al. } \\
2009 \text { study } 1\end{array}$ & $\begin{array}{l}\mathrm{N}=15(\mathrm{MF}) \\
\mathrm{M}=21.1 \pm 3.0 \mathrm{y}\end{array}$ & $\mathrm{CO}$ & $3 \mathrm{mg} / \mathrm{kg}$ & 12 & $\begin{array}{l}\text { coffee } M=24 \\
\text { cups } / d\end{array}$ & $\begin{array}{l}\text { CPT-AX*, } \\
\text { Stop Task* }\end{array}$ & 40 \\
\hline $\begin{array}{l}\text { Tieges et al. } \\
2009 \text { study } 2\end{array}$ & $\begin{array}{l}\mathrm{N}=17(\mathrm{MF}) \\
\mathrm{M}=20.9 \pm 2.3 \mathrm{y}\end{array}$ & $\mathrm{CO}$ & $3 \mathrm{mg} / \mathrm{kg}$ & 12 & $\begin{array}{l}\text { coffee } M=24 \\
\text { cups } / \mathrm{d}\end{array}$ & Stop Task* & 40 \\
\hline
\end{tabular}




\begin{tabular}{|c|c|c|c|c|c|c|c|}
\hline Study & Sample & $\begin{array}{l}\mathbf{P A} / \\
\mathrm{CO}\end{array}$ & $T^{1}$ & $\mathbf{A}^{2}$ & $\begin{array}{l}\text { Habitual } \\
\text { caffeine } \\
\text { intake }^{3}\end{array}$ & $\begin{array}{l}\text { Attention } \\
\text { Task }^{4}\end{array}$ & Timing $^{5}$ \\
\hline $\begin{array}{l}\text { Tieges et al. } \\
2009 \text { study } 3\end{array}$ & $\begin{array}{l}\mathrm{N}=17(\mathrm{MF}) \\
\mathrm{M}=21.6 \pm 2.6 \mathrm{y}\end{array}$ & $\mathrm{CO}$ & $3 \mathrm{mg} / \mathrm{kg}$ & 12 & $\begin{array}{l}\text { coffee } M=24 \\
\text { cups } / \mathrm{d}\end{array}$ & $\begin{array}{l}\text { Flanker } \\
\text { Task* }\end{array}$ & 40 \\
\hline $\begin{array}{l}\text { Warburton et } \\
\text { al. } 1995\end{array}$ & $\begin{array}{l}\mathrm{N}=18(\mathrm{MF}) \\
18-30 \mathrm{y}\end{array}$ & $\mathrm{CO}$ & 75,150 & 24 & $\begin{array}{l}\text { coffee } M>3 \\
\text { cups } / d\end{array}$ & RVIP* & 45 \\
\hline $\begin{array}{l}\text { Yeomans et } \\
\text { al. } 2002\end{array}$ & $\begin{array}{l}\mathrm{N}=30(\mathrm{MF}) \\
\mathrm{M} \approx 22 \mathrm{y}\end{array}$ & $\mathrm{CO}$ & $\begin{array}{l}1,2 \\
\mathrm{mg} / \mathrm{kg}\end{array}$ & 10 & $\mathrm{M} \approx 330 \mathrm{mg} / \mathrm{d}$ & RVIP* & $\begin{array}{ll}\mathrm{t} 145 \\
\mathrm{t} 2105 / \mathrm{T} 1 \\
\mathrm{t} 3 & 45 / \mathrm{T} 2\end{array}$ \\
\hline
\end{tabular}

${ }^{1} \mathrm{PA} / \mathrm{CO}=$ parallel $/$ crossover design; N.A. = not available; ${ }^{2}$ caffeine dose reported in $\mathrm{mg}$, unless reported as $\mathrm{mg} / \mathrm{kg}$ (estimated by assuming a body weight of 70kg); ${ }^{3}$ caffeine abstinence reported in hours; ${ }^{4}$ habitual caffeine consumption reported per day (/d) or per week (/w); ${ }^{5}$ ANT = Attention Network Task; CFF = Critical Flicker Fusion; $C R T=$ Choice Reaction Time; CRT-FA = CRT focussed attention; CRT CS = CRT categoric search, $D V=$ Digit Vigilance, RVIP = Rapid Visual Information Processing; RDD = Repeat digit detection; SRT = Simple Reaction Time; TOVA = Test of Variables of Attention; * significant response for at least one of the tasks outcome measures; ${ }^{6}$ time reported in minutes post treatment (T), i.e. XX/T1 
Table 2 Overview of tasks, task classification and results per task (Hypothesis 1a and Hypothesis 2)

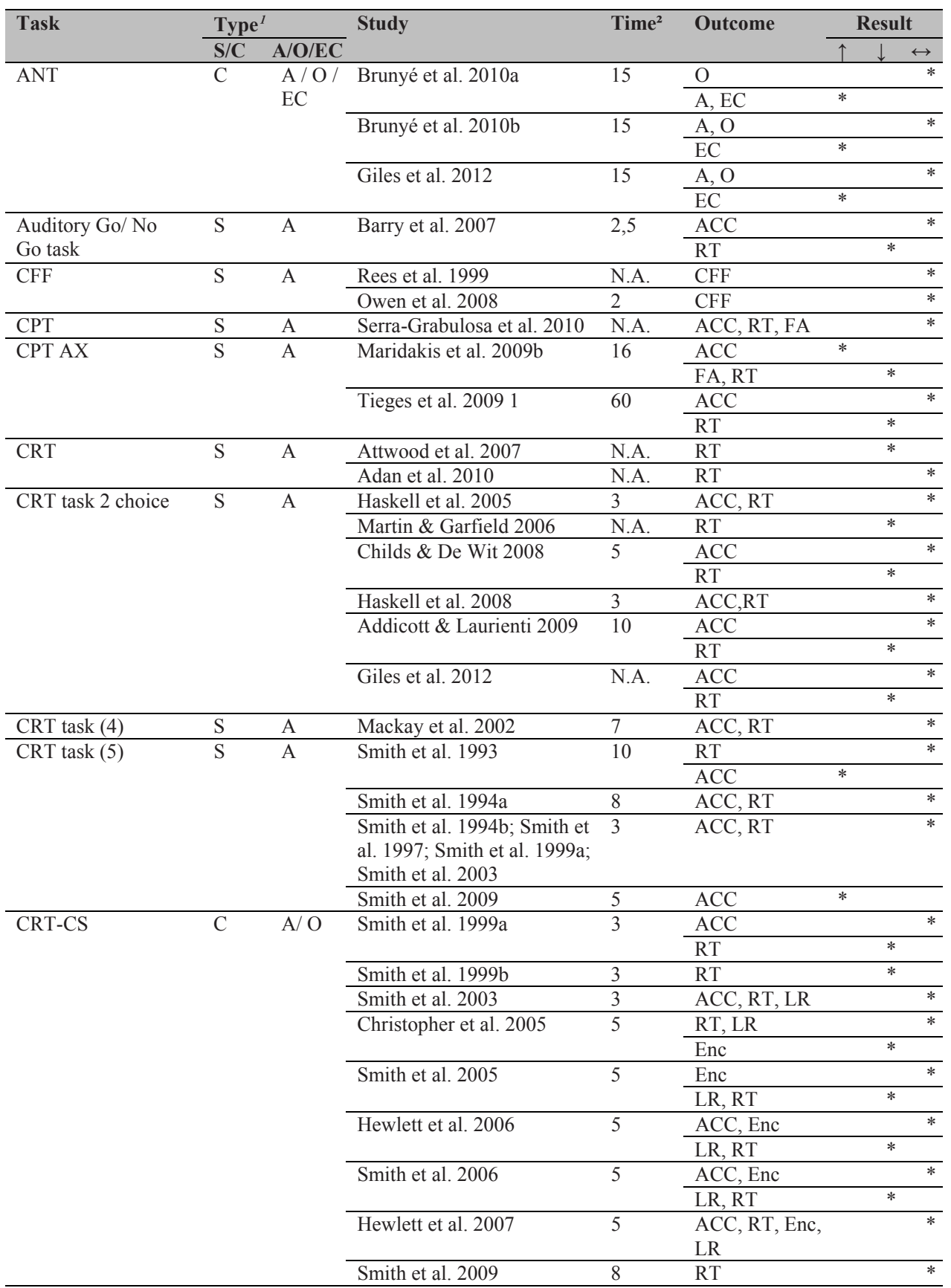




\begin{tabular}{|c|c|c|c|c|c|c|c|c|}
\hline \multirow[t]{2}{*}{ Task } & \multicolumn{2}{|c|}{ Type $^{I}$} & \multirow[t]{2}{*}{ Study } & \multirow[t]{2}{*}{ Time $^{2}$} & \multirow[t]{2}{*}{ Outcome } & \multicolumn{3}{|c|}{ Result } \\
\hline & $\mathrm{S} / \mathrm{C}$ & $\mathrm{A} / \mathrm{O} / \mathrm{EC}$ & & & & $\uparrow$ & & $\leftrightarrow$ \\
\hline \multirow[t]{15}{*}{ CRT-FA } & \multirow[t]{15}{*}{$\mathrm{S}$} & \multirow[t]{15}{*}{ A } & \multirow[t]{2}{*}{ Smith et al. 1999a } & \multirow[t]{2}{*}{3} & $\mathrm{ACC}$ & & & $*$ \\
\hline & & & & & RT & & * & \\
\hline & & & Smith et al. 2003 & 4 & $\mathrm{ACC}, \mathrm{RT}$ & & & $*$ \\
\hline & & & \multirow[t]{2}{*}{ Christopher et al. 2005} & \multirow[t]{2}{*}{5} & RT, Enc & & & $*$ \\
\hline & & & & & LR & & * & \\
\hline & & & \multirow[t]{2}{*}{ Smith et al. 2005} & \multirow[t]{2}{*}{5} & RT, LR & & & $*$ \\
\hline & & & & & Enc & $*$ & & \\
\hline & & & \multirow[t]{2}{*}{ Heatherly et al. 2005} & \multirow[t]{2}{*}{8} & RT & & & $*$ \\
\hline & & & & & $\mathrm{ACC}$ & $*$ & & \\
\hline & & & Hewlett et al. 2006 & 5 & ACC, RT, LR & & & $*$ \\
\hline & & & & & Enc & $*$ & & \\
\hline & & & Smith et al. 2006 & 5 & RT, LR & & & $*$ \\
\hline & & & & & $\mathrm{ACC}$, Enc & $*$ & & \\
\hline & & & Hewlett et al. 2007 & 5 & $\begin{array}{l}\text { ACC, RT, } \\
\text { Enc }\end{array}$ & & & $*$ \\
\hline & & & Smith et al. 2009 & 8 & RT & & & $*$ \\
\hline Concentration task & $\mathrm{S}$ & $\mathrm{A}$ & Ruijter et al. 2000c & 10 & $\mathrm{ACC}, \mathrm{RT}$ & & & $*$ \\
\hline Digit Vigilance & $\mathrm{S}$ & $\mathrm{A}$ & Haskell et al. 2005; Haskell & N.A. & $\mathrm{ACC}$ & & & $*$ \\
\hline & & & et al. 2008 & & RT & & * & \\
\hline Flanker task & $\mathrm{C}$ & $\mathrm{EC}$ & Lorist \& Snel 1997 & N.A. & RT, FA & & & $*$ \\
\hline & & & Kenemans et al. 1998 & 60 & $\mathrm{ACC}$ & & & $*$ \\
\hline & & & & & RT & & * & \\
\hline & & & Tieges et al. 20093 & 60 & $\mathrm{ACC}$ & & & $*$ \\
\hline & & & & & RT & & $*$ & \\
\hline Flanker task & $\mathrm{C}$ & $\mathrm{EC}$ & Addicott \& Laurienti 2009 & 45 & $\mathrm{ACC}$ & & & $*$ \\
\hline (adapted) & & & & & Int, RT & & * & \\
\hline Inhibition task & $\mathrm{C}$ & $\mathrm{EC}$ & Heatherly et al. 2005 & N.A. & $\mathrm{ACC}$ & $*$ & & \\
\hline RVIP & $\mathrm{C}$ & $\mathrm{EC}$ & Hasenfratz et al. 1994 & 20 & $\mathrm{ACC}$ & & & $*$ \\
\hline & & & & & RT & & * & \\
\hline & & & Warburton et al. 1995 & 10 & $\mathrm{ACC}$ & $*$ & & \\
\hline & & & & & RT & & * & \\
\hline & & & Rees et al. 1999 & 15 & $\mathrm{ACC}, \mathrm{FA}$ & & & * \\
\hline & & & Smit \& Rogers 2000 & 10 & RT & $*$ & & \\
\hline & & & Yeomans et al. 2002 & 10 & $\mathrm{ACC}, \mathrm{RT}$ & $*$ & & \\
\hline & & & Haskell et al. 2005 & N.A. & $\mathrm{RT}, \mathrm{ACC}, \mathrm{FA}$ & & & $*$ \\
\hline & & & Haskell et al. 2008 & N.A. & RT, FA & & & $*$ \\
\hline & & & Owen et al. 2008 & 4 & $\mathrm{ACC}, \mathrm{RT}, \mathrm{FA}$ & & & $*$ \\
\hline & & & Maridakis et al. 2009a & 16 & FA & & $*$ & \\
\hline & & & Maridakis et al. 2009b & 16 & $\mathrm{ACC}, \mathrm{RT}, \mathrm{FA}$ & & $*$ & \\
\hline & & & Kennedy \& Haskell 2011 & 5 & $\mathrm{ACC}, \mathrm{RT}, \mathrm{FA}$ & & & $*$ \\
\hline & & & Attwood et al. 2007 & 6 & $\mathrm{ACC}, \mathrm{RT}, \mathrm{FA}$ & & & $*$ \\
\hline RDD & $\mathrm{S}$ & A & Smith et al. 1992 & 8 & $\mathrm{ACC}$ & $*$ & & \\
\hline & & & & & RT & & * & \\
\hline & & & Smith et al. 1994a & 8 & $\mathrm{ACC}$ & $*$ & & \\
\hline & & & & & FA, RT & & * & \\
\hline & & & Smith et al. 1994b & 3 & $\mathrm{ACC}$ & $*$ & & \\
\hline & & & & & RT & & * & \\
\hline & & & Smith et al. 1997 & 3 & $\mathrm{ACC}, \mathrm{RT}$ & & & $*$ \\
\hline & & & Smith et al. 1999a & 3 & $\mathrm{ACC}$ & & & $*$ \\
\hline & & & Brice \& Smith 2001 & 3 & RT & & & $*$ \\
\hline & & & & & $\mathrm{ACC}$ & $*$ & & \\
\hline & & & Smith et al. 2003 & 3 & $\mathrm{ACC}, \mathrm{FA}$ & & & $*$ \\
\hline & & & & & RT & & $*$ & \\
\hline & & & Christopher et al. 2005 & 5 & $\mathrm{ACC}$ & & & $*$ \\
\hline & & & & & RT & & $*$ & \\
\hline
\end{tabular}




\begin{tabular}{|c|c|c|c|c|c|c|c|c|}
\hline \multirow[t]{2}{*}{ Task } & \multicolumn{2}{|c|}{ Type $^{I}$} & \multirow[t]{2}{*}{ Study } & \multirow[t]{2}{*}{ Time $^{2}$} & \multirow[t]{2}{*}{ Outcome } & \multicolumn{3}{|c|}{ Result } \\
\hline & $\mathrm{S} / \mathrm{C}$ & $\overline{\mathrm{A} / \mathrm{O} / \mathrm{EC}}$ & & & & $\uparrow$ & $\downarrow$ & $\leftrightarrow$ \\
\hline & & & \multirow[t]{2}{*}{ Smith et al. 2005} & \multirow[t]{2}{*}{5} & RT & & & $*$ \\
\hline & & & & & $\mathrm{ACC}$ & $*$ & & \\
\hline & & & Hewlett et al. 2006 & 5 & HR, RT & * & & \\
\hline & & & Smith et al. 2006 & 5 & RT, FA & & & $*$ \\
\hline & & & \multirow[t]{2}{*}{ Hewlett et al. 2007} & \multirow[t]{2}{*}{5} & $\mathrm{ACC}$ & $*$ & & \\
\hline & & & & & RT & & 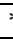 & \\
\hline & & & \multirow[t]{3}{*}{ Maridakis et al. 2009a } & \multirow[t]{3}{*}{16} & FA & & & $*$ \\
\hline & & & & & $\mathrm{ACC}$ & $*$ & & \\
\hline & & & & & RT & & & \\
\hline & & & \multirow[t]{2}{*}{ Maridakis et al. 2009b } & \multirow[t]{2}{*}{16} & FA, RT & & & \\
\hline & & & & & $\mathrm{ACC}$ & $*$ & & \\
\hline & & & \multirow[t]{2}{*}{ Smith et al. 2009} & \multirow[t]{2}{*}{8} & $\mathrm{ACC}$ & & & * \\
\hline & & & & & RT & & & \\
\hline Selection task & $\mathrm{C}$ & EC & Kenemans et al. 1995 & 15 & $\mathrm{ACC}$ & $*$ & & \\
\hline & & & & & RT & & & \\
\hline Selection task & $\mathrm{C}$ & $\mathrm{O} /$ & Ruijter et al. 2000a & 20 & RT & & & $*$ \\
\hline (colour) & & $\mathrm{EC}$ & & & $\mathrm{ACC}$ & * & & \\
\hline Selection task & $\mathrm{C}$ & $\mathrm{A}$ & Ruijter et al. $2000 \mathrm{~b}$ & 20 & RT & & & $*$ \\
\hline (spatial) & & & & & $\mathrm{ACC}$ & * & & \\
\hline Sequential RT & $\mathrm{S}$ & $\mathrm{A}$ & Adan et al. 2010 & N.A. & RT & & & $*$ \\
\hline SRT & $\mathrm{S}$ & $\mathrm{O}$ & Smith et al. 1993 & 10 & RT & & & \\
\hline & & & Smith et al. 1994a & 8 & RT & & & \\
\hline & & & Smith et al. $1994 b$ & 3 & RT & & & \\
\hline & & & Fine et al. 1994 & 10 & FA & & & $*$ \\
\hline & & & & & $\mathrm{ACC}$ & $*$ & & \\
\hline & & & & & RT & & & \\
\hline & & & Richardson et al. 1995 & 6 & RT & & & \\
\hline & & & Smith et al. 1997 & 30 & RT & & & $*$ \\
\hline & & & Rogers et al. 1998 & 10 & RT & & & \\
\hline & & & Robelin et al. 1998 & 20 & RT & & & \\
\hline & & & Rees et al. 1999 & N.A. & RT & & & \\
\hline & & & Smith et al. 1999a & 3 & RT & & & $*$ \\
\hline & & & Smit \& Rogers 2000 & 20 & RT & & & \\
\hline & & & Kelemen et al. 2001 & 12 & $\mathrm{ACC}$ & $*$ & & \\
\hline & & & & & RT & & & \\
\hline & & & Smith et al. 2003 & 3 & RT & & & $*$ \\
\hline & & & Christopher et al. 2005 & 5 & RT & & & $*$ \\
\hline & & & Smith et al. 2005 & 5 & RT & & & \\
\hline & & & Haskell et al. 2005 & N.A. & RT & & & \\
\hline & & & Heatherly et al. 2005 & 12 & RT & & & \\
\hline & & & Deslandes et al. 2005 & & $\mathrm{ACC}, \mathrm{RT}$ & & & $*$ \\
\hline & & & Hewlett et al. 2006 & 5 & RT & & & \\
\hline & & & Smith et al. 2006 & 5 & RT & & & \\
\hline & & & Martin \& Garfield 2006 & 8 & ACC, RT & & & $*$ \\
\hline & & & Childs \& De Wit 2006 & 20 & FA & & & $*$ \\
\hline & & & & & $\mathrm{ACC}$ & $*$ & & \\
\hline & & & & & RT & & & \\
\hline & & & Hewlett et al. 2007 & 5 & RT & & & $*$ \\
\hline & & & Attwood et al. 2007 & N.A. & RT & & & * \\
\hline & & & Childs \& De Wit 2008 & 5 & RT & & & \\
\hline & & & Haskell et al. 2008 & N.A. & RT & & & \\
\hline & & & Smith et al. 2009 & 8 & RT & & & $*$ \\
\hline & & & Adan et al. 2010 & N.A. & RT & & & $*$ \\
\hline & & & Giles et al. 2012 & N.A. & $\mathrm{ACC}$ & & & $*$ \\
\hline & & & & & RT & & 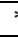 & \\
\hline
\end{tabular}




\begin{tabular}{|c|c|c|c|c|c|c|c|}
\hline \multirow[t]{2}{*}{ Task } & \multicolumn{2}{|c|}{ Type $^{I}$} & \multirow[t]{2}{*}{ Study } & \multirow[t]{2}{*}{ Time $^{2}$} & \multirow[t]{2}{*}{ Outcome } & \multicolumn{2}{|c|}{ Result } \\
\hline & $\mathrm{S} / \mathrm{C}$ & $\mathrm{A} / \mathrm{O} / \mathrm{EC}$ & & & & $\uparrow$ & $\leftrightarrow$ \\
\hline \multirow[t]{3}{*}{ Spatial cuing task } & \multirow[t]{3}{*}{$\mathrm{C}$} & \multirow[t]{3}{*}{$\mathrm{EC}$} & Kenemans et al. 1998 & N.A. & $\mathrm{ACC}, \mathrm{RT}$ & & * \\
\hline & & & \multirow[t]{2}{*}{ Kelly et al. 2008} & \multirow[t]{2}{*}{ N.A. } & $\mathrm{ACC}, \mathrm{D}^{\prime}$ & $*$ & \\
\hline & & & & & RT & & * \\
\hline \multirow[t]{3}{*}{ Stop task } & \multirow[t]{3}{*}{$\mathrm{C}$} & \multirow[t]{3}{*}{$\mathrm{EC}$} & Childs \& De Wit 2006 & N.A. & RT & & * \\
\hline & & & Tieges et al. 20092 & 60 & $\mathrm{ACC}$ & & * \\
\hline & & & Tieges et al. 20091 & 60 & RT & & $*$ \\
\hline \multirow[t]{4}{*}{ Stroop (original) } & \multirow[t]{4}{*}{$\mathrm{C}$} & \multirow[t]{4}{*}{$\mathrm{EC}$} & Schmitt et al. 2003 & N.A. & Int & & * \\
\hline & & & Deslandes et al. 2005 & N.A. & $\mathrm{ACC}, \mathrm{RT}$ & & * \\
\hline & & & Hasenfratz et al. 1992 & N.A. & Int & & $*$ \\
\hline & & & Kenemans et al. 19992 & N.A. & Int & & $*$ \\
\hline \multirow{2}{*}{$\begin{array}{l}\text { Stroop (number- } \\
\text { digit) }\end{array}$} & \multirow[t]{2}{*}{$\mathrm{C}$} & \multirow[t]{2}{*}{ EC } & \multirow[t]{2}{*}{ Kenemans et al. 19991} & \multirow[t]{2}{*}{ N.A. } & Int & & $*$ \\
\hline & & & & & RT & & * \\
\hline \multirow[t]{6}{*}{ Switch task } & \multirow[t]{6}{*}{$\mathrm{C}$} & \multirow{6}{*}{$\begin{array}{l}\mathrm{A} / \mathrm{O} / \\
\mathrm{EC}\end{array}$} & \multirow[t]{2}{*}{ Tieges et al. 2004} & \multirow[t]{2}{*}{90} & $\mathrm{ACC}$ & & $*$ \\
\hline & & & & & RT & & $*$ \\
\hline & & & \multirow[t]{2}{*}{ Tieges et al. 2006} & \multirow[t]{2}{*}{90} & $\mathrm{ACC}$ & & * \\
\hline & & & & & RT, Switch C & & $*$ \\
\hline & & & \multirow[t]{2}{*}{ Owen et al. 2008} & \multirow[t]{2}{*}{5} & RT, FA & & * \\
\hline & & & & & $\mathrm{ACC}$ & $*$ & \\
\hline \multirow[t]{2}{*}{ Switch task (cued) } & $\mathrm{C}$ & $\mathrm{A} / \mathrm{O} /$ & Tieges et al. 2007 & 90 & Switch RT & & $*$ \\
\hline & & EC & & & Switch ACC & & * \\
\hline TOVA & $\mathrm{C}$ & $\mathrm{A}$ & Hunt et al. 2011 & 22 & $\mathrm{ACC}$ & & * \\
\hline & & & & & RT & & $*$ \\
\hline Visual search task & $\mathrm{S}$ & $\mathrm{A}$ & Lorist et al. 1994; Lorist et al & N.A. & $\mathrm{ACC}$ & & * \\
\hline & & & 1996 & & RT & & $*$ \\
\hline
\end{tabular}

$A N T=$ Attention Network Task $; C F F=$ Critical Flicker Fusion $; C R T=$ Choice Reaction Time; CRT-FA $=C R T$ focussed attention; $C R T C S=C R T$ categoric search, RVIP = Rapid Visual Information Processing; RDD = Repeat digit detection; SRT = Simple Reaction Time; TOVA = Test of Variables of Attention

${ }^{1} S=$ simple attention, $C=$ complex attention; $A=$ alerting, $O=$ orienting $E C=$ executive control; ${ }^{2} T=$ task duration in minutes; ${ }^{3} A C C=$ accuracy of response, $R T=$ response time, $F A=$ false alarms, $H R=$ hit rate, $C F F=$ critical flicker fusion threshold, $D^{\prime}=d$ prime, End $=$ encoding, Int $=$ interference, $A=$ alerting, $O=$ orienting, EC = executive control, Switch $C=$ switch costs 
Table 3 Hypothesis 1b: The effect of caffeine consumption on complex attention processes is not solely dependent on its effects on simple attention processes

\begin{tabular}{|c|c|c|c|}
\hline \multirow[t]{2}{*}{ Study } & \multicolumn{2}{|c|}{ Task type } & \multirow[t]{2}{*}{ Results } \\
\hline & $\mathbf{S}$ & $\mathrm{C}$ & \\
\hline Addicott \& Laurienti, 2009 & $*$ & $*$ & Caffeine improved CRT as well as Flanker task response speed \\
\hline Attwood et al., 2007 & $*$ & & $\begin{array}{l}\text { Caffeine improved CRT response speed but not RVIP perfor- } \\
\text { mance }\end{array}$ \\
\hline Brunyé et al., 2010 a & $*$ & * & Caffeine improved ANT alerting and executive control \\
\hline Brunyé et al., 2010 b & & $*$ & Caffeine improved ANT ex. control but not alerting \\
\hline Childs \& De Wit, 2006 & $*$ & & $\begin{array}{l}\text { Caffeine improved SRT accuracy and response speed but not Stor } \\
\text { task performance }\end{array}$ \\
\hline Christopher et al. 2005 & $*$ & $*$ & $\begin{array}{l}\text { Caffeine improved CRT-FA long responses, RDD response speed } \\
\text { and CRT-CS encoding }\end{array}$ \\
\hline Deslandes et al., 2005 & & & Caffeine did not improve SRT or Stroop performance \\
\hline Gilles et al., 2012 & $*$ & $*$ & $\begin{array}{l}\text { Caffeine improved SRT and CRT response speed as well as ANT } \\
\text { ex. control but not alerting }\end{array}$ \\
\hline Haskell et al., 2005 & $*$ & & $\begin{array}{l}\text { Caffeine improved SRT response speed, CRT and Digit vigilance } \\
\text { accuracy and response speed, but not RVIP performance }\end{array}$ \\
\hline Haskell et al., 2008 & $*$ & & $\begin{array}{l}\text { Caffeine improved SRT response speed, CRT and Digit vigilance } \\
\text { accuracy and response speed, but not RVIP performance }\end{array}$ \\
\hline Heatherly et al., 2005 & $*$ & $*$ & $\begin{array}{l}\text { Caffeine improved SRT response speed, CRT-FA accuracy as } \\
\text { well as inhibition task accuracy }\end{array}$ \\
\hline Hewlett et al. 2006 & $*$ & * & $\begin{array}{l}\text { Caffeine improved CRT- FA encoding, RDD accuracy, SRT } \\
\text { response speed, and CRT-CS response speed }\end{array}$ \\
\hline Hewlett et al., 2007 & $*$ & & Caffeine improved RDD response speed but not CRT-FA/CS \\
\hline Maridakis et al., 2009a & $*$ & $*$ & $\begin{array}{l}\text { Caffeine improved RDD response speed and accuracy and RVIP } \\
\text { response speed and accuracy }\end{array}$ \\
\hline Maridakis et al., 2009b & $*$ & $*$ & $\begin{array}{l}\text { Caffeine improved CPT and RDD response speed and accuracy as } \\
\text { well as RVIP response speed and accuracy }\end{array}$ \\
\hline Owen et al., 2008 & & $*$ & $\begin{array}{l}\text { Caffeine improved Switch accuracy but not CFF or RVIP perfor- } \\
\text { mance }\end{array}$ \\
\hline Rees et al., 1999 & $*$ & * & $\begin{array}{l}\text { Caffeine improved SRT response speed as well as RVIP respons } \\
\text { speed but not CFF performance }\end{array}$ \\
\hline Smit \& Rogers, 2000 & $*$ & $*$ & Caffeine improved SRT response speed and RVIP accuracy \\
\hline Smith et al. 1999 & $*$ & * & Caffeine improved CRT-FA/CA speed but not RDD or SRT \\
\hline Smith et al. 2009 & $*$ & & $\begin{array}{l}\text { Caffeine improved RDD response speed, but not CRT (5), CRT- } \\
\text { FA/CS or SRT }\end{array}$ \\
\hline Smith et al., 2003 & $*$ & & $\begin{array}{l}\text { Caffeine improved CRT-FA and RDD response speed but not } \\
\text { CRT, SRT or CRT-CS }\end{array}$ \\
\hline Smith et al., 2005 & $*$ & $*$ & $\begin{array}{l}\text { Caffeine improved CRT-FA encoding, RDD accuracy, SRT } \\
\text { response speed and CRT-CS response speed }\end{array}$ \\
\hline Smith et al., 2006 & $*$ & $*$ & $\begin{array}{l}\text { Caffeine improved CRT-FA accuracy and encoding, SRT re- } \\
\text { sponse speed and CRT-CS response speed }\end{array}$ \\
\hline
\end{tabular}

ANT = Attention Network Task CFF = Critical Flicker Fusion $; C R T=$ Choice Reaction Time $;$ CRT-FA $=$ CRT focussed attention; $C R T C S=C R T$ categoric search, $R V I P=$ Rapid Visual Information Processing; RDD = Repeat digit detection; SRT = Simple Reaction Time; TOVA = Test of Variables of Attention 


\section{Table $4 \quad$ Hypothesis 3: A higher caffeine dose exerts stronger attention effects}

\begin{tabular}{|c|c|c|}
\hline Study & Treatment* & Results \\
\hline Smith et al., 1993 & $0,105,210$ & Significant caffeine effect; unrelated to dose \\
\hline Hasenfratz et al. 1994 & $\begin{array}{l}0,105,210 \\
420\end{array}$ & $\begin{array}{l}\text { Significant caffeine effect; partially dose-related: increased } \\
\text { processing rate after } 105 \text { and } 210 \mathrm{mg} \text { but not after } 420 \mathrm{mg} \text {; } \\
\text { decreased RT after } 105 \text { and } 450 \mathrm{mg} \text {; trend for decreased } \\
\text { commission errors after } 105 \mathrm{mg} \text { but increased after } 420 \mathrm{mg}\end{array}$ \\
\hline Richardson et al., 1995 & $0,70,250$ & $\begin{array}{l}\text { Significant caffeine effect; negatively dose-related; faster } \\
\text { only after } 70 \mathrm{mg}\end{array}$ \\
\hline Warburton et al. 1995 & $0,75,150$ & $\begin{array}{l}\text { Significant caffeine effect; positively dose-related: larger } \\
\text { increases in performance and larger decreases in reaction } \\
\text { times after } 150 \mathrm{mg}\end{array}$ \\
\hline Kenemans et al., 1998 & $0,105,210$ & Significant caffeine effect unrelated to dose \\
\hline Rogers et al., 1998 & $0,70,140$ & Significant caffeine effect; unrelated to dose \\
\hline Smit et al., 2000 & $\begin{array}{l}0,12.5,25 \\
50,100\end{array}$ & Significant caffeine effect; unrelated to dose \\
\hline Yeomans et al., 2002 & $0,70,140$ & Significant caffeine effect; unrelated to dose \\
\hline Tieges et al., 2004 & $0,210,350$ & $\begin{array}{l}\text { Significant caffeine effect; trend for positively dose-related: } \\
\text { fewer errors after } 210 \text { then } 350 \mathrm{mg}\end{array}$ \\
\hline Haskell et al., 2005 & $0,75,150$ & $\begin{array}{l}\text { Significant caffeine effect; dose-response varies per test/ test } \\
\text { outcome: Digit vigilance RT, unrelated to dose; Digit Vigi- } \\
\text { lance ACC positively dose-related; RVIP ACC negatively } \\
\text { dose related }\end{array}$ \\
\hline Smith et al., 2005 & $0,105,210$ & Significant caffeine effect; unrelated to dose \\
\hline Tieges et al., 2006 & $0,210,350$ & Significant caffeine effect; unrelated to dose \\
\hline Childs \& De Wit, 2006 & $\begin{array}{l}0,50,150 \\
450\end{array}$ & $\begin{array}{l}\text { Significant caffeine effect; partially dose-related: } 150,450 \\
\text { mg increased ACC, } 450 \mathrm{mg} \text { decreased RT }\end{array}$ \\
\hline Tieges et al., 2007 & $0,210,350$ & Significant caffeine effect; unrelated to dose \\
\hline Maridakis et al., 2009a & $0,100,200$ & Significant caffeine effect; positively dose-related \\
\hline Brunyé et al., 2010a & $\begin{array}{l}0,100,200 \\
400\end{array}$ & Significant caffeine effect; positively dose-related \\
\hline Brunyé et al., 2010b & $\begin{array}{l}0,100,200 \\
400\end{array}$ & Significant caffeine effect; positively dose-related \\
\hline
\end{tabular}

* in $\mathrm{mg}$; caffeine dose reported as $\mathrm{mg} / \mathrm{kg}$ are estimated by assuming a body weight of $70 \mathrm{~kg}$. 
Table 5 Hypothesis 4: Habitual caffeine consumers require a greater caffeine dose to experience attention effects

\begin{tabular}{|c|c|c|}
\hline Study & Habitual intake & Results \\
\hline Fine et al., 1994 & $\begin{array}{l}\text { High }>100 \mathrm{mg} \\
\text { Low }<100 \mathrm{mg}\end{array}$ & Significant caffeine effect; unrelated to habitual intake \\
\hline Richardson et al., 1995 & $\begin{array}{l}\text { High }>200 \mathrm{mg} \\
\text { Non-consumer }\end{array}$ & Significant caffeine effect; unrelated to habitual intake \\
\hline Smit \& Rogers, 2000 & $\begin{array}{l}\text { High }>200 \mathrm{mg} \\
\text { Low }<100 \mathrm{mg}\end{array}$ & $\begin{array}{l}\text { Significant caffeine effect; related to habitual intake: } \\
\text { only significant for high habitual consumers }\end{array}$ \\
\hline Haskell et al., 2005 & $\begin{array}{l}\text { High: } 217 \mathrm{mg} \\
\text { Non-consumer }\end{array}$ & Significant caffeine effect; unrelated to habitual intake \\
\hline Hewlett et al. 2006 & $\begin{array}{l}\text { High: } 200 \mathrm{mg} \\
\text { Low: } 50 \mathrm{mg} \\
\text { Non-consumer }\end{array}$ & $\begin{array}{l}\text { Significant caffeine effect; varies per task: unrelated } \\
\text { to habitual intake for SRT, DV, CRT-SA; fastest for } \\
\text { high habitual intake for CRT, CRT-FA }\end{array}$ \\
\hline Smith et al., 2006 & $\begin{array}{l}\text { Consumer } \\
\text { Non-consumer }\end{array}$ & $\begin{array}{l}\text { Significant caffeine effect; varies per task: unrelated } \\
\text { to habitual intake for SRT, DV, CRT-FA; fastest for } \\
\text { non-habitual intake for CRT-SA }\end{array}$ \\
\hline Hewlett et al., 2007 & $\begin{array}{l}\text { High: } 212 \mathrm{mg} \\
\text { Low-medium: } 53.6 \mathrm{mg} \\
\text { Non-consumer }\end{array}$ & Significant caffeine effect; unrelated to habitual intake \\
\hline Hunt et al., 2011 & $\begin{array}{l}\text { Medium-high }<120 \mathrm{mg} \\
\text { Low }>120 \mathrm{mg}\end{array}$ & $\begin{array}{l}\text { Significant caffeine effect; related to habitual intake: } \\
\text { significant for low habitual intake only }\end{array}$ \\
\hline $\begin{array}{l}\text { Kennedy \& Haskell, } \\
2011\end{array}$ & $\begin{array}{l}\text { High: } 333 \mathrm{mg} \\
\text { Low: } 6-67 \mathrm{mg}\end{array}$ & $\begin{array}{l}\text { No significant caffeine effect; unrelated to habitual } \\
\text { intake }\end{array}$ \\
\hline Attwood et al., 2007 & $\begin{array}{l}\text { High: }>200 \mathrm{mg} \\
\text { Low-medium }<200 \mathrm{mg}\end{array}$ & $\begin{array}{l}\text { Significant effects of caffeine; related to habitual } \\
\text { intake: significant for high habitual intake only }\end{array}$ \\
\hline
\end{tabular}

* in mg; caffeine doses reported as $\mathrm{mg} / \mathrm{kg}$ are estimated by assuming a body weight of $70 \mathrm{~kg}$.

Table 6 Hypothesis 5: Effects of caffeine consumption on attention are not influenced by withdrawal - non-withdrawn volunteers

\begin{tabular}{ll}
\hline Study & Results \\
\hline Smith et al. 1997 & No significant caffeine effect \\
\hline Christopher et al. 2005 & Significant caffeine effect: faster on RDD, CRT-CS \\
\hline Smith et al., 2005 & Significant caffeine effect: faster on SRT, CRT-CS/FA, more accurate on \\
& RDD \\
\hline Childs \& De Wit, 2006 & Significant caffeine effect: faster and more accurate on SRT \\
\hline Childs \& De Wit, 2008 & Significant caffeine effect: faster on SRT, CRT \\
\hline Addicott \& Laurienti, 2009 & Significant caffeine effect: faster on CRT, Flanker task \\
\hline
\end{tabular}


Table 7 Hypothesis 5: Effects of caffeine consumption on attention are not influenced by withdrawal - briefly withdrawn volunteers

\begin{tabular}{lll}
\hline Study & Abstinance (in hrs) & Results \\
\hline Smith et al. 1994b & 1 & Significant caffeine effect \\
\hline Richardson et al., 1995 & $1 \frac{1}{2}$ & Significant caffeine effect \\
\hline Rees et al., 1999 & 2 & Significant caffeine effect; faster on SRT, RVIP \\
\hline Smith et al. 1999a & 2 & $\begin{array}{l}\text { Significant caffeine effect; faster on CRT-FA/CS, } \\
\text { RVIP }\end{array}$ \\
\hline
\end{tabular}

Table 8 Hypothesis 5: Effects of caffeine consumption on attention are not influenced by withdrawal - various abstinence levels

\begin{tabular}{lll}
\hline Study & Abstinance (in hrs) & Results \\
\hline Heatherly et al., 2005 & $4,6,8$ & $\begin{array}{l}\text { Significant caffeine effect; partially related to absti- } \\
\text { nence level per task/ outcome: strongest effect on } \\
\text { CRT, SRT, inhibition task after 8hr abstinence }\end{array}$ \\
\hline Addicott \& Laurienti, 2009 & 0,30 & $\begin{array}{l}\text { Significant caffeine effect; partially related to absti- } \\
\text { nence level per task/ outcome: faster on CRT after } \\
\text { abstinence; unrelated for Flanker task }\end{array}$ \\
\hline Richardson et al., 1995 & $11 / 2,12,7$ days & Significant caffeine effect; unrelated to abstinence \\
\hline
\end{tabular}

Table 9 Hypothesis 5: Effects of caffeine consumption on attention are not influenced by withdrawal - repeated caffeine consumption

\begin{tabular}{lll}
\hline Study & Treatment* & Results \\
\hline Warburton et al. 1995 & $\begin{array}{l}\text { Preload: } 75 \\
2^{\text {nd }} \text { dose: } 0,75,150\end{array}$ & Significant caffeine effect; added effect of $2^{\text {nd }}$ dose \\
\hline Robelin \& Rogers, 1998 & $\begin{array}{l}\text { Preload: } 0,84 \\
2^{\text {nd }} \text { dose: } 0,84\end{array}$ & $\begin{array}{l}\text { Significant caffeine effect; no added effect of } 2^{\text {nd }} \text { or } \\
3^{\text {rd }} \text { dose }\end{array}$ \\
& $3^{\text {rd }}$ dose: 0,84 & \\
\hline Yeomans et al., 2002 & Preload: $0,70,140$ & Significant caffeine effect; no added effect of $2^{\text {nd }}$ dose \\
& $2^{\text {nd }}$ dose: $0,70,140$ & \\
\hline Smith et al., 2005 & Preload: 0,105 & \\
& $2^{\text {nd }}$ dose: 0,105 & Significant caffeine effect; added effect of $2^{\text {nd }}$ dose \\
\hline Hewlett et al. 2007 & Preload: 0,70 & Significant caffeine effect; added effect of $2^{\text {nd }}$ dose \\
& $2^{\text {nd }}$ dose: 0,70 & \\
\hline
\end{tabular}

* in mg; caffeine doses reported as $\mathrm{mg} / \mathrm{kg}$ are estimated by assuming a body weight of $70 \mathrm{~kg}$ 



\section{L-theanine \& caffeine improve task switching but}

not intersensory attention or subjective alertness alertness. Appetite, 54, 406-9. 


\section{ABSTRACT}

Tea ingredients L-theanine and caffeine have repeatedly been shown to deliver unique cognitive benefits when consumed in combination. The current randomized, placebocontrolled, double-blind, cross-over study compared a combination of L-theanine $(97 \mathrm{mg})$ and caffeine $(40 \mathrm{mg}$ ) to a placebo on two attention tasks and a self-report questionnaire before, and 10 and 60 minutes after consumption. The combination of L-theanine and caffeine significantly improved attention on a switch task as compared to the placebo, while subjective alertness and intersensory attention were not improved significantly. The results support previous evidence that L-theanine and caffeine in combination can improve attention. 


\section{INTRODUCTION}

Tea is the second most widely consumed beverage in the world, only being surpassed by water. Traditionally, tea consumption has been associated with mental clarity (Graham, 1992). While anecdotal reports of the cognitive effects of tea are abundant, scientists have only recently started to accumulate evidence for these effects using cognitive tasks (Hindmarch, Quinlan, Moore, \& Parkin, 1998; Hindmarch, Rigney, Stanley, Quinlan, Rycroft et al., 2000).

The cognitive effects of tea are commonly attributed to two of its naturally occurring components: caffeine and L-theanine or theanine (Bryan, 2008). A typical serving of black tea contains approximately 35 to $61 \mathrm{mg}$ caffeine per $200 \mathrm{~mL}$. Apart from a general arousing effect, caffeine has been reported to enhance cognitive performance, in particular attention (Smith, 2002). Caffeine, in doses ranging from the equivalent to a cup of tea up to 200-250 mg, has been shown to improve performance on a range of attention measures, such as vigilance, visual search, choice reaction time and selective attention (Christopher et al., 2005; Durlach, 1998; Fagan, Swift, \& Tiplady, 1988; Lieberman, Wurtman, Emde, Roberts, \& Coviella, 1987; Lorist et al., 1996; Ruijter, Lorist, \& Snel, 1999; Ruijter et al., 2000b; Smith et al., 1999a). Although few studies have found more pronounced effects of $500 \mathrm{mg}$ caffeine (Bruce, Scott, Lader, \& Marks, 1986; Ruijter et al., 1999), most studies indicate that effects of caffeine on performance seem to asymptote around $200 \mathrm{mg}$ (Lieberman et al., 1987; Robelin \& Rogers, 1998). In addition to improved performance, caffeine has been shown to affect self-reported mental state, in particular alertness (Robelin \& Rogers, 1998; Christopher et al., 2005; Fagan et al., 1988; Mumford, Evans, Kaminski, Preston, Sannerud et al., 1994), concentration (Mumford et al., 1994) and energetic arousal (Quinlan et al., 1997; Quinlan et al., 2000).

Beside caffeine, tea includes the nonproteinic amino acid theanine. Theanine is found almost exclusively in tea leaves, with a typical serving of black tea containing 4.5 to $22.5 \mathrm{mg}$ per $200 \mathrm{~mL}$. The effects of theanine on cortical activity have been investigated with electroencephalography (EEG) at rest and during task execution. Theanine was found to increase EEG alpha activity at rest, indicating more relaxation (Juneja, 
Chu, Okubi, Nagato, \& Yokogoshi, 1999; Nobre, Rao, \& Owen, 2008), as well as background alpha desynchronisation and attention-related alpha synchronisation when preparing to focus attention on specific stimuli (Gomez-Ramirez, Higgins, Rycroft, Owen, Mahoney et al., 2007). At the behavioural level however, effects of theanine are equivocal. While Rogers, Smith, Heatherley, and Pleydell-Pearce (2008) reported no significant improvements following $200 \mathrm{mg}$ theanine, Haskell, Kennedy Milne, Wesnes, and Scholey (2008) found that $250 \mathrm{mg}$ improved choice reaction time and reaction times on two memory tasks. However, theanine also decreased serial subtraction performance and self-reported alertness.

As tea is the most prominent source of theanine and it is thus nearly universally consumed in combination with caffeine, studies investigating theanine and caffeine in combination are essential to our understanding of the effects of tea. Furthermore, these studies have provided evidence for an interaction between caffeine and theanine. For example, theanine protects against toxic caffeine doses in the rat brain in vitro (Kimura, Kurita, \& Murata, 1975) and inhibits excitatory effects of caffeine (Kakuda, Nozawa, Unno, Okamura, \& Okai, 2000; Kimura et al., 1975).

More recently, evidence for this interaction emerged from human studies in which the amount of theanine was increased relative to caffeine to amplify possible effects of theanine. Kelly, Gomez-Ramirez, Monetesi and Foxe (2008) found that 100 $\mathrm{mg}$ theanine in combination with 50mg caffeine improved accuracy on a visuo-spatial attention task and decreased background alpha activity as measured with EEG as compared to placebo, indicating improved attentional processing, whereas neither theanine nor caffeine alone differed from placebo. Haskell, Kennedy, Milne, Wesnes, and Scholey (2008) reported that $250 \mathrm{mg}$ theanine in combination with $150 \mathrm{mg}$ caffeine significantly improved sentence verification, simple reaction time, numeric working memory, and delayed word recognition reaction times, as compared to placebo, while caffeine or theanine in isolation did not. Moreover, the theanine/ caffeine combination significantly increased feelings of alertness, as compared to placebo. In a study by Owen, Parnell, De Bruin, and Rycroft (2008), both $100 \mathrm{mg}$ theanine and $50 \mathrm{mg}$ caffeine in combination and 50mg caffeine alone improved accuracy on a switch task at 60 minutes and 90 minutes after consumption respectively. There were no treatment effects on response speed. Finally, a similar theanine/ caffeine combination improved switch task 
accuracy, increased self-reported alertness, and reduced task-induced fatigue as compared to placebo (Giesbrecht et al., 2010).

The studies described above indicate that theanine and caffeine improve cognitive performance, attention in particular, and that these effects are most pronounced when both ingredients are combined. The current study aimed to further investigate the enhancing effects of theanine and caffeine in combination. Specifically, the aim of this study was twofold. Firstly, we wanted to replicate the effect on the switch task (Owen et al., 2008). Secondly, as the switch task assessed visual attention, we wanted to investigate whether this effect was dependent on perceptual modality by including a paradigm which required switching attention between different modalities and has previously been shown to be sensitive to the effects of theanine (Gomez-Ramirez et al., 2007). Previous studies which included theanine and/or caffeine in isolation (Haskell et al., 2008; Kelly et al., 2008; Owen et al., 2008) indicated that the theanine/ caffeine combination had synergistic effects. Moreover, theanine is virtually exclusively consumed in combination with caffeine. Therefore it was decided to specifically compare the effects of the theanine/ caffeine combination to a placebo. In line with prior studies, we increased the amount of theanine, relative to caffeine, to amplify possible effects of theanine. 


\section{METHODS}

\section{Participants}

Twenty-nine healthy participants (11 males), aged 18 to 45 years $(M=30.6, S D=8.9)$ took part in the study. Inclusion criteria were regular caffeinated tea and/or coffee consumption, non-smoking, regular breakfast consumption, and a BMI between 20 and 30 . The participants consumed on average $11.7(S D=6.7)$ caffeinated drinks per week and had a mean BMI of $25.1(S D=2.6)$. Exclusion criteria were allergies to caffeine, artificial sweeteners, or herbal supplements, colour blindness, dyslexia, pregnancy, breastfeeding, and use of recreational drugs and medication with the exception of the contraceptive pill. The study was approved by the Unilever Colworth Research Ethics Committee. Participants signed an informed consent form and were paid for their participation.

\section{Design}

The study employed a randomized, placebo-controlled, double-blind, crossover design with drink (the active theanine/ caffeine drink vs. a placebo) and time (cognitive test battery at baseline, 10 minutes post-drink and 60 minutes post-drink) as within participant factors. The order of drinks was randomized. Participants attended two test occasions, separated by at least 6 and no more than 14 days, during which they consumed either the active drink or the placebo and completed a test battery three times. Each test occasion was preceded by a brief training session to minimize learning effects.

\section{Drinks}

The active drink was prepared by dissolving synthetically produced theanine (Suntheanine $^{\mathrm{TM}}$; Taiyo Kagaku Co. Ltd., Yokkaichi, Japan), pharmaceutical grade caffeine and iced tea powder into $500 \mathrm{~mL}$ of mineral water. The iced tea powder contained colourings and tea flavourings, as well as sweeteners (sucralose) and lemon flavour to mask the taste of caffeine. The placebo drink matched the active drink except for the absence of theanine and caffeine. The active drink delivered on average $97 \mathrm{mg}$ theanine and $40 \mathrm{mg}$ caffeine. 


\section{Cognitive test battery}

Each 40-minute session consisted of two tests of attention and a mood questionnaire. Tests are described in the order in which they were performed.

\section{Intersensory attention task (Foxe, Simpson, \& Ahlfors, 1998)}

This task measures the ability to selectively deploy attention to stimuli presented in different sensory modalities (visual and auditory) and it was chosen to investigate whether the effect of the theanine/ caffeine combination is dependent on the visual modality as opposed to a general attention effect. This intersensory attention task has been shown to be sensitive to the effects of theanine (Gomez-Ramirez et al., 2007). During each trial, a cue was presented which instructed participants to which modality they would need to attend to. This cue was followed by a pair of visual stimuli (Gabor patches differing in orientation) and/or auditory stimuli (tones differing in pitch), with a stimulus onset asynchrony of $1200 \mathrm{~ms}$. Stimuli were presented in isolation (unisensory) or in combination (intersensory). Participants were required to perform a discrimination task on stimuli in the cued modality. The intertrial interval randomly varied between 1400 and $2400 \mathrm{~ms}$. Total task duration was 30 minutes.

\section{Switch task (Rogers \& Monsell, 1995)}

The switch task measures executive control, particularly the ability to shift back and forth between different task sets, i.e., task switching (Monsell, 2003). This task has been shown to be sensitive to the effects of the theanine/ caffeine combination (Owen et al., 2008). During each trial, a letter and number were simultaneously presented in red or purple. The letter was randomly drawn from a set of four vowels (A, E, I, U) and four consonants $(\mathrm{G}, \mathrm{K}, \mathrm{M}, \mathrm{R})$, the number from a set of four even $(2,4,6,8)$ and four odd numbers $(3,5,7,9)$. If the letter and number stimuli were presented in purple, participants were required to press spacebar if an even number was presented; if presented in red, they were required to respond if a vowel was presented. In all other cases participants were to withhold their response. Stimulus colours and the associated task set changed every three trials. Intertrial intervals were $1700 \mathrm{~ms}$. Total task duration was 10 minutes. 
Bond-Lader visual analogue scales (Bond \& Lader, 1974)

Bond-Lader visual analogue scales (VAS) comprise of 16 bipolar items consisting of adjective pairs (e.g. Tense/Relaxed). Participants use a $100 \mathrm{~mm}$ line to indicate how they feel on the dimension defined by two adjectives. These VAS have been shown to possess sound psychometric properties and factor analysis has revealed three distinct factors: alertness, contentedness, and calmness. Furthermore, the scales have been found to be sensitive to the effects of theanine and caffeine in combination (Haskell et al., 2008; Kelly et al., 2008; Owen et al., 2008).

\section{Procedure}

Participants arrived at $9.00 \mathrm{am}$, after abstaining from caffeine-containing products, alcohol, and mushrooms (some boletes contain theanine) from $9.00 \mathrm{pm}$ the previous day. Compliance with restrictions was checked by means of a self-report checklist. Participants then completed a baseline measurement, followed by a 10 minute break during which they consumed the drink, and participated in two test sessions starting 10 and 60 minutes post-drink.

\section{Analysis}

Subjective mood scores and reaction times on the attention tests were analyzed using a 2 x 2 mixed model ANOVA with drink (active drink vs. placebo) and session (10-50 minutes post-drink vs. 60-100 minutes post-drink) as within-subject factors. The proportion of correct responses were analysed with a logistic regression-based Generalised Linear Mixed Model, with aforementioned within-subject factors. For the switch task, trial type $\left(1^{\text {st }}, 2^{\text {nd }}, 3^{\text {rd }}\right.$ trial after a switch between task sets) and response type (correct hit, correct withhold) were additional within-subject factors.

All analyses employed baseline scores and drink order as covariates. Statistical tests were two-sided with alpha $=0.05$. In case of significant main or interaction effects involving treatment, the analysis was rerun with habitual caffeine consumption as an additional covariate. Standard error and degrees of freedom were estimated by the Kenward-Roger method. 


\section{RESULTS}

\section{Intersensory attention task}

On the unisensory auditory subtask, there was a trend for a main effect of drink on reaction times $(F(1,23.2)=3.58, p=0.071)$ with participants tending to respond faster after the active drink as compared to placebo. On the intersensory visual subtask, there was a tendency for a drink by session interaction for reaction times $(F(1,52)=3.21, p=0.079)$ indicating that after the active drink but not after placebo, participants tended to respond faster at 60-100 minutes post-drink. The proportion of correct responses did not differ significantly between conditions.

\section{Switch task}

Mean accuracy for both drinks 10-50 minutes and 60-100 minutes post-drink, is presented in Figure 1. There was a main effect of $\operatorname{drink}(F(1,24.56)=10.71, p=0.003)$ indicating a higher proportion of correct responses after the active drink as compared to placebo. This effect remained significant after including habitual caffeine intake as a covariate, indicating that the improvement in number of correct responses was not simply due to relief of caffeine withdrawal. Furthermore, there was a significant interaction between drink and response type $(F(1,665)=18.38, p<0.001)$ indicating a more pronounced effect of the active drink on correct hits $(t(38.3)=4.52, p<0.0001)$ than on correct withholds $(t(29.9)$ $=1.41$, n.s.). Worthy of note is that as the proportion of correct withholds was not impaired by the drink, a shift in response bias cannot account for the effect on correct hits. Reaction times did not differ significantly between conditions. This indicates that the effect of the active drink on accuracy is not due to a speed/accuracy trade-off. The drink by trial type $\left(1^{\text {st }}, 2^{\text {nd }}, 3^{\text {rd }}\right.$ trial after a switch between task sets $)$ interaction was not significant, signifying that the active drink did not affect switch costs.

\section{Bond-Lader Visual Analogue Scales}

Participants tended to feel more alert after placebo compared to the active drink, evidenced by a trend towards a main effect of drink $(F(1,22.5)=3.82, p=0.063)$. Selfrated calmness and contentedness did not show an effect of drink. 


\section{DISCUSSION}

The main findings of the present study can be summarized as follows. First, this study provided further evidence that the theanine/ caffeine combination improves attention on the switch task (Owen et al., 2008). The improvement in accuracy in combination with unaltered response speed is in line with previous studies (Giesbrecht et al., 2010; Kelly et al., 2008). Second, tentative evidence was found that the effect of the theanine/ caffeine combination on attention is not specific to the visual modality, as we found faster responses in both the visual and auditory modality on the intersensory task after the combination as compared to placebo. Third, improvements in attention as signified by the behavioural tasks were not associated with enhanced subjective alertness. This was not in line with previous studies, where theanine and caffeine in combination increased subjective alertness as compared to placebo (Giesbrecht et al., 2010; Haskell et al., 2008; Owen et al., 2008).

The finding that theanine and caffeine in combination clearly improved task switching while intersensory attention was only marginally improved raises questions about the specificity of the attention effect of theanine and caffeine. In the context of Posner's model of attention, which discriminates between three aspects of attention (i.e., alerting, orienting, and executive control; Posner \& Petersen, 1990; Heckman et al., 2010) the switch task is considered an example of a task assessing executive control as it involves response inhibition. However, as switch costs were not affected by the drink, it could be also argued that alerting (i.e. sensitivity to incoming target stimuli) was improved rather than executive control.

The intersensory attention task on the other hand, is considered to predominantly tap into the orienting aspect of attention as it involves the selection of specific information from multiple sensory inputs. The current results therefore seem to suggest that the theanine/ caffeine combination improves executive control/alerting rather than orienting. To the best of our knowledge, self-reported alertness has not been characterized in terms of alerting, orienting and executive control, hence precluding interpretation of drink effects on self-reported alertness in the light of Posner's model. 
These interpretational ambiguities notwithstanding, evidence indicates that the theanine/ caffeine combination influences attention. The findings cannot be accounted for by a general increase in arousal, as demonstrated by the lack of improvement in selfreported alertness. Notably, this is in contrast to the effect of caffeine in isolation. By and large there is consensus that caffeine improves basic cognitive functions (e.g., simple reaction times, vigilance) by increasing arousal via modulating adenosine receptor activation in the brain, while leaving higher-order functions unaffected (Smith, 2002). Although two recent studies have shown that a high dose of caffeine may improve aspects of executive control (Brunye et al., 2010b; Tieges et al., 2006), the totality of the evidence is equivocal. Thus, whereas caffeine predominantly yields arousing effects, a high dose of theanine may counteract these effects, therefore allowing for improved performance on higher-order tasks as employed in the current study. A case in point is that although caffeine is known to increase blood pressure (James, 2004; Tieges et al., 2006), a high dose of theanine counteracted these arousing effects (Rogers et al., 2008).

The main limitation of the present study is that it did not include theanine or caffeine in isolation and we therefore cannot conclude that their combination produces greater attention benefits, than one would be able to achieve with theanine or caffeine in isolation. Yet, the robust synergistic effects of theanine with caffeine found in previous studies make this very likely (Haskell et al., 2008; Kelly et al., 2008; Owen et al., 2008).

In conclusion, the present study showed that the theanine/ caffeine combination improved attention as measured with the switch task, and to a lesser degree the intersensory attention task. Taken together with the previous studies, we conclude that a high dose of theanine combined with caffeine at the level of a single cup of tea, can improve attention. 


\section{FIGURES}

Fig. 1

Proportion of correct responses on the switch task

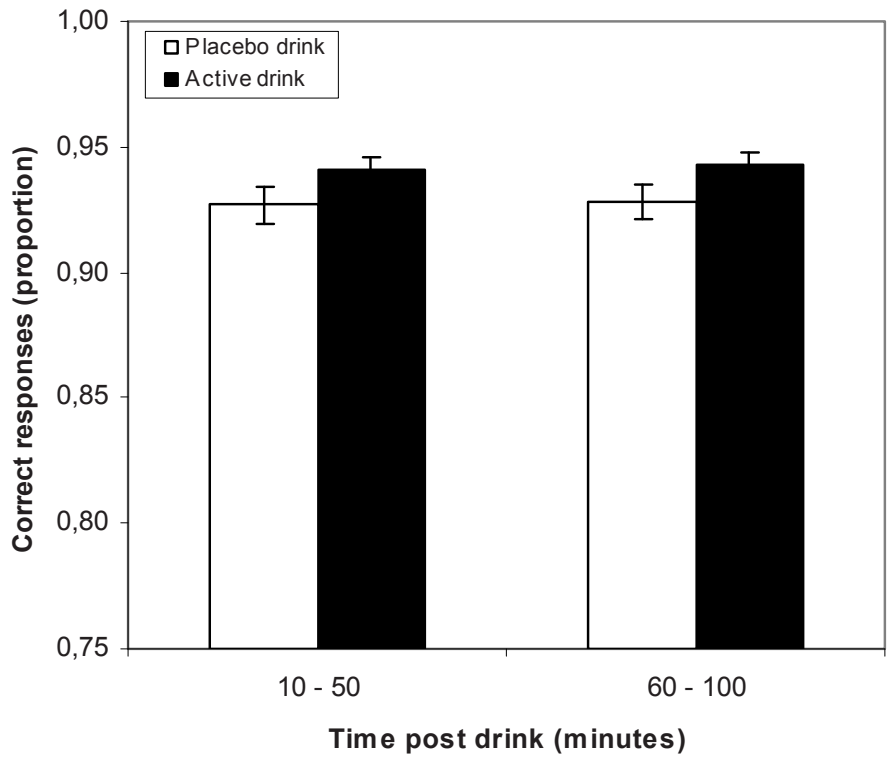




\section{ABSTRACT}

Tea has historically been associated with mood and performance benefits, such as relaxation and concentration. This review summarizes the research on the acute effects of tea, and its ingredients theanine and caffeine, on attention and mood. Consistent with abundant research on the benefits of caffeine, the performance benefits of tea were identified in a number of studies, with particularly consistent evidence for improved attention. Tea consumption also consistently improved self-reported alertness and arousal, while effects on pleasure or relaxation are less consistent. In addition to the research on caffeine in real-life performance, two recent studies have provided a broader perspective on tea's effects on psychological function, as they showed beneficial effects in related areas such as work performance and creativity. These studies demonstrate the validity of laboratory findings by supporting the idea that tea consumption has acute benefits on both mood and performance in real-life situations. 


\section{INTRODUCTION}

Whereas consumers have historically associated tea with mental benefits, these alleged benefits were largely anecdotal until relatively recently. In the past 15 years, the effects of tea consumption on mental performance, especially attention, and mood, have been investigated in a number of studies. Notably, the studies reviewed here mainly pertain to relatively acute effects of tea and tea ingredients, which occur immediately after consumption or during the course of a day, in healthy adult populations.

The timing of the occurrence of these benefits contrasts with the research on neuroprotective effects of tea employing longitudinal designs, which have suggested long term benefits of tea consumption in elderly or impaired populations (Song et al., 2012). Whereas the literature on tea's acute psychological benefits focuses on outcome measures such as attention and mood, the primary focus of the literature on tea's chronic health benefits lies on prevention of cardiovascular disease (Ras, Zock, \& Draijer, 2011; Kim, Chiu, Barone, Avino, Wang et al., 2011; Zheng, Xu, Li, Liu, Hui et al., 2011), diabetes (Huxley, Lee, Barzi, Timmermeister, Czernichow et al., 2009; Jing, Han, Hu, Bi, Li et al., 2009), depression (Niu, Hozawa, Kuriyama, Ebihara, Guo et al., 2009; Feng et al., 2012) and neurodegenerative diseases like dementia (Song et al., 2012). The underlying mechanisms for acute and chronic benefits are likely to be different; the acute effects have been ascribed to caffeine and theanine, whereas the chronic effects have been linked to other components in tea, such as flavonoids. Based on knowledge currently available, the different outcome measures and different underlying mechanisms appear to hinder linking tea's acute benefits to tea's chronic benefits. In this paper, we mainly focus on the acute benefits of tea on attention and mood.

In September 2012, relevant studies were identified through searches of the PubMed and Scopus electronic databases using the entry terms 'tea', 'caffeine', or 'theanine' in combination with 'attention', 'concentration', 'alertness', 'cognition', 'cognitive function', or 'mood'. We limited our search to journal articles written in English. To be included, studies were required to investigate the effects of tea, caffeine and/or theanine, report data on at least one acute behavioural measure of attention or mood, include at least one (sub)sample of healthy adults, include a control condition, 
and have been published in a peer-reviewed journal. Relevance was assessed based on the titles and abstracts. Finally, relevant papers that appeared until April 2013 in the electronic databases of Pubmed and Scopus were included. 


\section{TEA AND ATTENTION}

According to the American Psychological Association, attention is "a state of focused awareness on a subset of the available perceptual information". Attention allows the brain to effectively deal with the vast amount of input that is continuously received through its sensory (e.g., vision, hearing) and cognitive processes (e.g. memory) and to focus on what is relevant. As such, attention is an important prerequisite for many cognitive processes, including memory and reasoning. Attention can be measured objectively as performance on attention tests, usually in terms of speed of response and number of correct responses, or subjectively as self-reported alertness, usually by means of a visual analogue rating scale (Westenhoefer, Bellisle, Blundell, de, Edwards et al., 2004).

\section{Acute effects of Tea Ingredients on Attention}

Tea contains a large number of bioactive compounds, yet its attention benefits have generally been attributed to two components, caffeine and theanine (Bryan, 2008). Typically, a cup of tea contains 35 to $61 \mathrm{mg}$ caffeine and 4.5 to $22.5 \mathrm{mg}$ theanine. Other ingredients in tea, such as the green tea polyphenol epigallocatechin gallate (EGCG), have been ascribed certain neuroprotective effects (Scholey, Downey, Ciorciari, Pipingas, Nolidin et al., 2012), but acute effects on performance measures have not been found (Wightman, Haskell, Forster, Veasey, \& Kennedy, 2012).

\section{Caffeine}

There is a large body of evidence that caffeine is highly bio-available, being rapidly and almost completely absorbed (Magkos \& Kavouras, 2005), and readily distributed throughout all tissues of the body (Blanchard \& Sawers, 1983) including the brain (Dager \& Friedman, 2000). Peak plasma concentrations are reached about 30 minutes post consumption, and half-lives for elimination range between 2.5 to 10 hours, dependent on the food matrix, genetic factors, and the habit of smoking (Magkos \& Kavouras, 2005; Seng, Fun, Law, Lim, Fan et al., 2009). Caffeine is extensively metabolized in the 
liver to more than 25 derivatives, while considerably less than $5 \%$ of the ingested dose is excreted unchanged in the urine (Magkos \& Kavouras, 2005).

Caffeine is known to affect neurotransmission in general, by antagonizing (i.e. competing with) adenosine receptors. When adenosine binds to its receptors in the brain, primarily A1 and A2a receptors, neural activity slows down. However, when caffeine binds instead to these receptors it causes a general increase in neurotransmission (Fisone et al., 2004; Daly, Jacobson, \& Ukena, 1987; Smith, 2005a). As such, caffeine attenuates the inhibitory effects of adenosine in the brain. As an adenosine antagonizer, caffeine also affects the dopaminergic system (Ferre, Fredholm, Morelli, Popoli, \& Fuxe, 1997) which is involved in arousal and higher-order attentional processes (Fan et al., 2005). In addition, caffeine has secondary effects on the release of specific neurotransmitters including noradrenaline, acetylcholine, serotonin, glutamate and gamma-aminobutyric acid (GABA) which have been hypothesized to be involved in caffeine's effect on arousal (Nehlig, Daval, \& Debry, 1992).

Caffeine is present in a range of beverages (e.g. tea, coffee, soft drinks, energy drinks) and in some foods (e.g. chocolate), and is the most consumed psychoactive ingredient worldwide (Ferre, 2008). Its attention effects have been studied extensively (Einother \& Giesbrecht, 2013; Rogers, 2007). Across a large variety of tests and doses, these studies indicate that even in low doses such as $50 \mathrm{mg}$ (i.e., roughly the equivalent of a cup of tea) caffeine improves performance on attention tasks and subjective alertness. Whereas effects on simple attention tasks have been well-established, recent studies have also indicated beneficial effects of caffeine on more complex multifaceted attention tasks (Brunye et al., 2010a; Brunye et al., 2010b; Tieges et al., 2006).

\section{Theanine}

Theanine is an amino acid that is virtually unique to tea (apart from the fungus Bay bolete). Theanine has been shown to be highly bioavailable, reaching its maximum concentration in plasma about 45 minutes post-administration (Van der Pijl et al., 2010). It is metabolized into glutamic acid and ethylamine, and a minor fraction is excreted into urine (Scheid, Ellinger, Alteheld, Herholz, Ellinger et al., 2012; Terashima, Takido, \& Yokogoshi, 1999; Van der Pijl et al., 2010). 
Upon administration of theanine, levels of some other amino acids (e.g. tryptophan, tyrosine) were reduced in the brain in rats (Yokogoshi, Kobayashi, Mochizuki, \& Terashima, 1998). Some of these amino acids are precursors of neurotransmitters important for cognitive performance (e.g. tryptophan is converted into serotonin, and tyrosine into dopamine), which are both involved in higher order complex attention processes in humans (serontonin: Canli, Omura, Haas, Fallgatter, Constable et al., 2005; Canli, Qui, Omura, Condon, Haas et al., 2006; dopamine: Kakuda, Nozawa, Sugimoto, \& Niino, 2002). Hence, it has been suggested that theanine may attenuate neurotransmitter levels by interfering with the availability of their precursors and thus affect cognition. In addition, theanine can bind to receptors and transporters involved in glutamate and GABA neurotransmission (Kakuda et al., 2002; Kakuda, Hinoi, Abe, Nozawa, Ogura et al., 2008), neurotransmitters which are also involved in attention performance (Robbins \& Murray, 2006; Levin, Bushnell, \& Rezvani, 2011).

For theanine, the psychoactive properties appear more complex than caffeine. During rest, theanine increases alpha wave brain activity, which has been associated with relaxation (Juneja et al., 1999; Nobre et al., 2008). During attention task performance, theanine increased background (resting) alpha activity, yet decreased alpha activity when preparing to attend to the task stimuli (Gomez-Ramirez et al., 2007; Gomez-Ramirez, Kelly, Montesi, \& Foxe, 2009), which has been related to better performance (Klimesch, Doppelmayr, Russegger, Pachinger, \& Schwaiger, 1998). Behavioural effects of theanine were largely absent. However, these studies were designed to measure brain activity rather than behavioural effects.

As caffeine and theanine are always consumed together when consuming tea, studying the effects of these ingredients in combination is more relevant than studying either ingredient in isolation. Few studies have investigated this combination, and most of them employed an increased level of theanine, as compared to the amount typically present in tea. This combination improved speed and accuracy on a range of tasks from the Cognitive Drug Research (CDR) test battery (Haskell et al., 2008), improved accuracy on an Attention Switching test (Einother, Martens, Rycroft, \& De Bruin, 2010; Giesbrecht et al., 2010; Owen et al., 2008), improved accuracy on a cued attention test (Kelly et al., 2008), and improved accuracy on a sustained attention test (Foxe, Morie, Laud, Rowson, De Bruin et al., 2012). 


\section{Acute effects of Tea on Attention}

The evidence on tea ingredients indicates that tea can provide cognitive benefits, in particular in the domain of attention. Four studies to date have specifically assessed the effects of black tea on attention performance (See Table 1). Hindmarch and colleagues (1998) compared the effects of coffee, water, and tea with and without $100 \mathrm{mg}$ caffeine on two tasks that are frequently used to measure attention effects in pharmaceutical trials, the Critical Flicker Fusion Threshold (CFFT) and Choice Reaction Time (CRT) tasks, and self-reported alertness. Overall, caffeinated beverages improved performance and selfreported alertness as compared to decaffeinated beverages. Interestingly, performance on the CFFT improved more after caffeinated tea than after caffeinated water, suggesting that the attention benefit cannot be wholly attributed to caffeine, and that other tea components also contributed to the benefit. Furthermore, while performance naturally declined over time, this decline was attenuated by beverage type, in that tea was associated with a smaller decrease (i.e., better performance) than water. Thus, this study provided tentative evidence for a beneficial effect of tea on a performance measure related to attention.

In a follow-up study, main effects of caffeine $(37.5 \mathrm{mg}$ and $75 \mathrm{mg}$ ) on both tasks were replicated (Hindmarch et al., 2000). Moreover, CFFT performance was improved after consumption of tea with $75 \mathrm{mg}$ caffeine, as compared to coffee with the same amount of caffeine. In sum, these findings suggest that tea effects - at least on simple attention tasks - are not merely due to caffeine and that on some outcomes tea might “outperform" coffee. Notably, both studies were of an open label design and did not control for expectations and taste differences between the beverages.

Two recent studies employed a double blind, placebo controlled, cross-over design and more complex attention tasks to further investigate these effects (De Bruin et al., 2011). In the first study, 26 healthy participants consumed two cups of black tea and placebo tea (coloured and flavoured water) on separate test sessions. After each cup, participants completed two complex tasks of attention (attention switching task and intersensory attention task) and a self-rating of alertness, calmness, and contentment. Results indicated that accuracy on the attention switching task was improved after tea compared to placebo. Moreover, participants also provided more accurate responses on two out of the four subtasks of the inter-sensory attention task and also responded faster on one of these two. Finally, participants felt more alert and less calm after tea consumption. 
In a replication of this study, participants consumed three cups of tea of a slightly weaker tea blend followed by the same tasks as the previous study after each cup. Again, accuracy on the attention switching task was improved after black tea as compared to placebo, and participants also reported feeling more alert. Tea did not significantly affect performance on the inter-sensory attention task. The authors speculated that this difference between the studies could be due to the difference in the strength of the tea blend and the potentially dose-dependent build-up of the active ingredients in plasma.

From the totality of research on tea summarized above, it can be concluded that consumption of black tea may improve attention and self-reported alertness. These conclusions are supported by studies on caffeine, and theanine and caffeine in combination. 


\section{TEA AND MOOD}

The influence of food and beverage consumption on mood has been (and still is) widely researched. Mood refers to a state of mind ranging from increased happiness, contentment, relaxation, alertness and energy, relief of depression and anxiety, to feelings of guilt and failure (Appleton \& Rogers, 2004). The consumption of tea, both black and green, has been associated with relaxation and refreshment (Graham, 1992; Shimbo et al., 2005) and feelings of satisfaction (Desmet \& Schifferstein, 2008). These putative benefits may be the result of the interaction of a number of elements, including the hot temperature at which tea is consumed, its sensory properties (e.g., smell, colour, mouthfeel), and its active ingredients which exert effects at different times during and after tea consumption.

In the studies summarized below, the effects of tea or tea ingredients on mood have been investigated using various validated scales with self-reports (e.g., BondLader visual analogue scales (BL VAS), Profile of Mood States (POMS), UWIST Mood Adjective Checklist (UMACL). These include facets of mood related to arousal and relaxation, as well as aspects related to the pleasantness of mood. In addition, particular changes in mood related to arousal and alertness can also be determined with physiological measures, for example, blood pressure and skin conductance (Mauss \& Robinson, 2009).

\section{Acute effects of Tea Ingredients on Mood}

Caffeine is well known for its effects on feelings of arousal, energy, and alertness (Nehlig, 2010; Ruxton, 2008) even at doses as low as $50 \mathrm{mg}$, which is comparable to the caffeine level in a cup of tea. In addition, a number of studies also found improved hedonic tone (Smith et al., 1999a; Heatherley et al., 2005), happiness and calmness (Warburton, 1995) and contentment (Amendola, Gabrieli, \& Lieberman, 1998) after caffeine consumption.

The number of studies on the effects of theanine in relation to mood is limited. Three studies found benefits in relation to stress; $200 \mathrm{mg}$ theanine reduced self-reported anxiety in anticipation of a stressful event (Lu, Gray, Oliver, Liley, Harisson et al., 
2013) or after a mental task (Yoto, Motoki, Murao, \& Yokogoshi, 2012) and reduced physiological indices of stress such as heart-rate and salivary immunoglobin during engagement in a stressful task (Kimura, Ozeki, Juneja, \& Ohira, 2007). Another study found that $250 \mathrm{mg}$ caffeine increased self-rated alertness, jitteriness, and blood pressure, with $200 \mathrm{mg}$ theanine antagonizing caffeine's effect on blood pressure but not its effects on alertness or jitteriness (Rogers, Smith, Heatherley, \& Pleydell-Pearce, 2008). Although psychological health benefits (including lower risk of cognitive decline) have been ascribed to tea flavonoids in cross-sectional studies (Ng, Feng, Niti, Kua, \& Yap, 2008) there is limited evidence for acute effects of these ingredients in healthy adults. Notably, the catechin EGCG has recently been associated with increased calmness and reduced stress in one study employing a $300 \mathrm{mg}$ dose (Scholey et al., 2012) but not in another study in which a lower dose of $135 \mathrm{mg}$ was used (Wightman et al., 2012).

\section{Acute effects of Tea on Mood}

A range of studies have assessed the effect of tea on mood (See Table 2). However, most of these studies focused on alertness or arousal, which is closely related to attention. Findings consistently demonstrate an increase in self-reported alertness or arousal after consuming black tea (and other caffeinated beverages) compared to noncaffeinated beverages. Five studies found reduced ratings of sedation on a Line Analogue Rating Scale (LARS) ratings scales, reflecting higher alertness, immediately after tea consumption (Aspen \& Quinlan, 1998) or 30 and 60 min after consumption of caffeinated beverages including tea (Hindmarch et al., 1998; Hindmarch et al., 2000; Quinlan et al., 2000) compared to non-caffeinated beverages. In addition, three studies found increased ratings of energetic arousal after consumption of caffeinated beverages, including tea, as compared to non-caffeinated beverages (Quinlan et al., 2000; Quinlan et al., 1997). Two studies reported increased alertness ratings after black tea consumption as compared to placebo on the Bond-Lader VAS (De Bruin et al., 2011). In a recent study, no effect of tea on arousal (using an Affect Grid) as compared to water was found (Einother, Baas, Rowson, \& Giesbrecht, 2015); in contrast to most of the previously mentioned studies, mood was assessed immediately after consumption. Findings from physiological measures, such as skin conductance and blood pressure, which are collected under controlled laboratory settings, further support that black tea can stimulate 
the autonomous nervous system in a manner associated with increased arousal (Hindmarch et al., 1998; Hindmarch et al., 2000).

Regarding valence of mood or pleasure, findings with tea are less consistent. Quinlan et al. (1997) found improved hedonic tone after drinking any beverage (i.e., black tea, coffee, hot water) at 30 and 60 minutes after consumption, compared to a condition with no drink at all. In a follow-up study, the researchers again found improved hedonic tone, but now after all caffeinated beverages, including black tea, as compared to non-caffeinated beverages (Hindmarch et al., 2000), while others found no significant effects of black tea consumption on contentment (De Bruin et al., 2011; Quinlan et al., 2000). All of these studies focused on the effects of the active ingredients which are expected to be absorbed and distributed to the brain relatively soon after consumption. An alternative hypothesis not driven by active ingredients, might be that mood is affected around and/or immediately after consumption due to the "tea consumption experience" (including the combination of the activity of preparing the tea, its sensory properties, etcetera), a hypothesis not tested in any of the studies described above.

Finally, in the study by Einother et al (2015) tea consumption (as well as a positive control) positively affected pleasantness of mood immediately after consumption, as compared to water. To date, no studies have assessed the acute effects of tea on selfreported relaxation. However, two studies have attempted to relate tea consumption to stress relief. Steptoe and colleagues (Steptoe, Gibson, Vuononvirta, Williams, Hamer et al., 2007) investigated the effects of daily tea consumption during six weeks in healthy men. While tea consumption did not affect blood pressure or heart rate, it did reduce platelet activation before and after stress, reduced cortisol levels, and increased subjective relaxation at the end of the recovery period, reflecting a positive effect of tea on recovery from stress. In addition, cross-sectional data from healthy Japanese adults showed that a high consumption of green tea ( $>5$ cups daily) was related to lower psychological distress, even after adjustment for possible confounders (Hozawa, Kuriyama, Nakaya, Ohmori-Matsuda, Kakizaki et al., 2009).

While caffeine is known to affect various aspects of mood, in particular alertness, effects of other ingredients such as theanine or EGCG on mood are largely unknown. The effects of tea on self-reported alertness and arousal have been well 
researched and have consistently shown a positive effect, consistent with the findings for caffeine. In contrast, only few studies have addressed the effect of tea on pleasure/hedonic tone and/or relaxation, and their results are variable. 


\section{EFFECTS OF TEA IN REAL LIFE: BEYOND ATTENTION AND MOOD}

Laboratory interventions using validated measures indicated that tea consumption exerts acute positive effects on attention performance and aspects of mood. Although such findings have good internal reliability, generalizability to the complex cognitive demands of everyday life has been questioned. Few studies investigated whether benefits could be extended here.

\section{Effects of Tea Ingredients in Real Life}

The performance benefits of caffeine have been identified in real life tasks. For example, caffeine is recognized by the US military as an "alertness-enhancing compound" (Caldwell \& Caldwell, 2005) and has been shown to improve vigilance during military combat (McLellan, Kamimori, Voss, Bell, Cole et al., 2005b; McLellan, Kamimori, Bell, Smith, Johnson et al., 2005; McLellan, Kamimori, Voss, Tate, \& Smith, 2007). Beneficial effects of caffeine consumption on simulated and/or actual driving are commonly found under extreme circumstances, such as following sleep deprivation (Horne, Reyner, \& Barrett, 2003; Reyner \& Horne, 2002a; Reyner \& Horne, 2002b) or during night time driving (Philip, Taillard, Moore, Delord, Valtat et al., 2006). Moreover, two studies have shown benefits in non-fatigued subjects during daytime, on outcomes corresponding to safer and more stable driving. Caffeine consumption $(3 \mathrm{mg} / \mathrm{kg})$ improved steering accuracy during a 1 hour simulated drive (Brice \& Smith, 2001). Furthermore, $80 \mathrm{mg}$ caffeine consumed after 2 hours of monotonous simulated driving improved lane keeping and speed maintenance during a 2 hour follow-up drive (Mets, Baas, Van Boven, Olivier, \& Verster, 2012). Others found no significant effects of caffeine (1.2 $\mathrm{mg} / \mathrm{kg}$ ) on steering variability, but this study may have been underpowered (Heatherley, Hayward, Hill, Smit, Cater et al., 2004).

Furthermore, a review of 13 studies of shift work performance showed that caffeine consumption can reduce errors and improve cognitive performance in shift workers, as compared to no intervention (Ker, Edwards, Felix, Blackhall, \& Roberts, 2010). Finally, others have indicate that caffeine $(100 \mathrm{mg})$ improved self-reported concentra- 
tion while attending a university lecture as compared to placebo (Peeling \& Dawson, 2007) and improved passing accuracy during a simulated soccer game $(6 \mathrm{mg} / \mathrm{kg})$ (Foskett, Ali, \& Gant, 2009). No studies to date have assessed effects of theanine or other tea ingredients on real-life tasks.

\section{Effects of Tea in Real Life}

Recent meta-analyses have confirmed that positive mood states lead to improved creative problem solving compared to neutral mood states (Baas, De Dreu, \& Nijstad, 2008). As tea has been shown to affect mood, it was hypothesized that consumption of tea and tea-based beverages would enhance creativity via positive affect. Isen and colleagues (Isen, Labroo, \& Durlach, 2004) reported that tasting a cup of commercially available or branded iced tea led to more creative problem solving on an adapted Remote Associate Test (RAT) as compared to tasting bottled water and compared to no intervention. This finding was confirmed by through a study in which hot black tea was found to positively affect pleasantness of mood immediately after consumption, as well as creative problem solving (Einother et al., 2015). Specifically, both tea and a validated positive affect induction (a procedure for recalling happy personal memories) were found to increase feelings of pleasantness as compared to water immediately after consumption. Furthermore, there was an indication that both positive affect inducing conditions improved creative problem solving as compared to water, in that both tended to yield faster insights on difficult problems.

In addition, two cross-sectional studies have assessed associations between consumption of tea (and other beverages) and performance and mood employing a naturalistic design. Steptoe et al. (2007) tested participants from two high-stress occupation groups who completed daily reports of beverage consumption and mood for 8 weeks and found relation between tea consumption and mood. In contrast, Bryan and colleagues (2012) asked professional and academic staff to complete reports of beverage consumption and mood and work performance during 10 working days. Their results indicated that participants who consumed more tea felt less tired and reported that they performed well at work more often than participants who drank less tea - but only if it was consumed without milk and/or sugar. In addition, higher consumption of non- 
caffeinated beverages was associated with feeling more relaxed. Coffee did not have a stronger relation than other beverages with any of the variables assessed. 


\section{CONCLUSIONS AND FUTURE DIRECTIONS}

Tea is one of the most consumed beverages in the world and its many putative benefits appear well-known and appreciated by consumers. We have summarized the acute psychological benefits of tea on attention and mood in an effort to link some of these anecdotal beliefs to the existing science. The tea ingredients caffeine and theanine, alone or in combination, have been linked to attention, with the available research showing that consumption of black tea improves attention on validated complex tasks as well as selfreported alertness. Mood benefits of tea and tea ingredients, other than improved alertness, have been less extensively researched until now and these findings are less consistent. The psychological benefits of tea and tea ingredients have been extended to some real life areas including driving, creativity and work performance.

Research on the benefits of tea is promising for attention and alertness, although questions remain regarding the scope and magnitude of impact as well as the sensitivity of different individuals. Whereas the bio-availability of both caffeine and theanine has been established (Blanchard \& Sawers, 1983; Dager \& Friedman, 2000; Scheid et al., 2012; Magkos \& Kavouras, 2005; Seng et al., 2009; Terashima et al., 1999; Van der Pij1 et al., 2010) as well as the (suggested) mechanisms of action in the brain of caffeine (Fan et al., 2005; Daly et al., 1987; Ferre et al., 1997; Smith, 2005a; Fisone et al., 2004; Nehlig et al., 1992) and theanine (Canli et al., 2005; Canli et al., 2006; Kakuda et al., 2002; Kakuda et al., 2008; Levin et al., 2011; Robbins \& Murray, 2006; Yokogoshi, Mochizuki, \& Saitoh, 1998) the extent to which they actually cross the Blood Brain Barrier (BBB) in humans and how much this is associated with (individual) changes in subsequent performance and mood measures is as yet unknown. It would be interesting to conduct a study (in humans) measuring cerebrospinal fluid concentrations of caffeine and theanine after acute ingestion of tea, and to correlate the concentrations with the acute effects on attention, mood and real life performance.

Other cognitive processes, such as memory or specific aspects of memory (e.g., working memory), that encompass a large component of the attention domain, have not yet been investigated in relation to acute effects of tea consumption. Indeed, very few studies have been conducted outside of the laboratory and it would be highly relevant to 
better understand the effects of tea consumption on other daily activities requiring attention, for example driving and cooking. Similarly, few studies have objectively assessed the acute effects of tea on pleasant feelings and relaxation, attributes which are often associated with each other tea by tea consumers. Investigating mood states during and after consumption of tea with validated measures will further build our understanding of the potential benefit of tea in this aspect of health and well-being.

Research on the benefits of caffeine on driving and other types of real-life performance is consistent with results from laboratory studies regarding improved alertness, reduced fatigue, and sustained performance benefits. Recent research provides tentative support for the hypothesis that benefits from tea can be found in related areas such as creative problem solving. Similarly, a cross-sectional study has shown that tea consumption improves self-reported work performance and fatigue. These results also warrant replication and further research. Nevertheless, the available evidence on the benefits of tea and tea ingredients on mood and performance in real-life settings does support the validity of findings from earlier laboratory-controlled studies on attention and mood. 


\section{TABLES}

\section{Table 1 Acute effects of tea consumption on attention ${ }^{1}$}

\begin{tabular}{|c|c|c|c|}
\hline Study & Sample & Design $^{2}$ & Results $^{3}$ \\
\hline $\begin{array}{l}\text { Hindmarch, } \\
1998\end{array}$ & $\begin{array}{l}\mathrm{N}=19 \\
10 \mathrm{~F} \\
\mathrm{M}=29.2 \mathrm{y}\end{array}$ & $\begin{array}{l}\text { R CO, } 5 \text { conditions: } \\
\text { - black tea, } 0 / 100 \mathrm{mg} \mathrm{C} \\
\text { - coffee, } 100 \mathrm{mg} \mathrm{C} \\
\text { - } \text { water, } 0 / 100 \mathrm{mg} \mathrm{C} \\
\end{array}$ & $\begin{array}{l}\text { CFFT: Overall, tea improved performance vs water } \\
\quad(\mathrm{p}=0.034) \text {; acutely, caffeinated beverage improved } \\
\text { CFFT level vs non-caffeinated beverages }(\mathrm{p}=0.024) \\
\text { CRT: no significant effects }\end{array}$ \\
\hline $\begin{array}{l}\text { Hindmarch, } \\
2000\end{array}$ & $\begin{array}{l}\mathrm{N}=30 \\
15 \mathrm{~F} \\
\mathrm{M}=27.3 \mathrm{y}\end{array}$ & $\begin{array}{l}\text { R CO, } 5 \text { conditions: } \\
\text { - black tea, } 37.5 / 75 \mathrm{mg} \mathrm{C} \\
\text { - coffee, } 75 / 150 \mathrm{mg} \mathrm{C} \\
\text { - hot water, } 0 \mathrm{mg} \mathrm{C}\end{array}$ & $\begin{array}{l}\text { CFFT: Caffeinated beverages resulted in superior per- } \\
\text { formance overall }(\mathrm{p}=0.0376) \text { and immediately after } \\
\text { drink } 1(\mathrm{p}=0.0425) \text { vs water; tea improved perfor- } \\
\text { mance } 30-90 \text { min after drink } 1(\mathrm{p}<0.01) \text { vs coffee. } \\
\text { CRT: Caffeinated beverages improved RRT after drink } \\
2 \text { vs water }(\mathrm{p}=0.0486) \text {; coffee improved RRT } \\
(\mathrm{p}=0.0484) \text { after drink } 2 \text {, vs tea }\end{array}$ \\
\hline $\begin{array}{l}\text { De Bruin, } \\
2011 \\
\text { Study } 1\end{array}$ & $\begin{array}{l}\mathrm{N}=26 \\
20 \mathrm{~F} \\
\mathrm{M}=30.7 \mathrm{y}\end{array}$ & $\begin{array}{l}\text { R PC CO, } 2 \text { conditions: } \\
\text { - black tea } \\
\text { - placebo tea }\end{array}$ & $\begin{array}{l}\text { Attention Switch task: improved accuracy after tea }(\mathrm{p} \\
\quad<.002) \\
\text { Intersensory attention task: improved accuracy on } \\
\text { multisensory auditory and visual tasks after tea } \\
\quad \text { (resp. } \mathrm{p}<0.001, \mathrm{p}<0.030) \text { and faster responses to } \\
\quad \text { visual task }(\mathrm{p}=0.043)\end{array}$ \\
\hline $\begin{array}{l}\text { De Bruin, } \\
2011 \\
\text { Study } 2\end{array}$ & $\begin{array}{l}\mathrm{N}=32 \\
15 \mathrm{~F} \\
\mathrm{M}=30.3 \mathrm{y}\end{array}$ & $\begin{array}{l}\text { R PC CO, } 2 \text { conditions: } \\
\text { - black tea } \\
\text { - placebo tea }\end{array}$ & $\begin{array}{l}\text { Attention Switch task: Improved accuracy after tea } \\
\qquad(\mathrm{p}=.007) \\
\text { Intersensory attention task: no significant effects }\end{array}$ \\
\hline
\end{tabular}




\section{Table 2 Acute effects of tea consumption on self-reported mood}

\begin{tabular}{rll}
\hline Study & Sample & Design $^{1}$ \\
\hline Aspen, & $\mathrm{N}=16$ & R CO, 7 conditions: \\
1998 & $8 \mathrm{~F}$, & - black tea, swallowed / \\
& $\mathrm{M}>18 \mathrm{y}$ & rinsed \\
& & - caffeinated water, swal- \\
& & lowed / rinsed \\
& - water, swallowed/ rinsed \\
& & (no caffeine) \\
& & no drink control
\end{tabular}

Results $^{2}$

LARS:

- <10min: more alert, energetic, attentive, clearheaded, refreshed and less tired $(\mathrm{p}<.05)$ after tea vs caffeinated water, regardless of rinse or swallow; relaxed, calm, tense, sad, happy unaffected

30min: more alert, energetic, attentive, clearheaded, refreshed and less tired $(\mathrm{p}<.01)$ after caffeine if swallowed; more calm, relaxed and less tense after tea vs caffeinated water, and caffeinated beverages vs water $(\mathrm{p}<.05)$; more happy, less sad after caffeinated beverages $(\mathrm{p}<.01)$

\begin{tabular}{|c|c|c|}
\hline $\begin{array}{l}\text { Quinlan, } \\
1997\end{array}$ & $\begin{array}{l}\mathrm{N}=16 \\
8 \mathrm{~F} \\
\mathrm{M}=35,2 \mathrm{y}\end{array}$ & 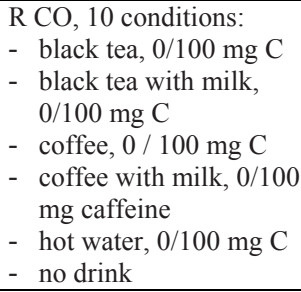 \\
\hline $\begin{array}{l}\text { Hindmarch, } \\
1998\end{array}$ & $\begin{array}{l}\mathrm{N}=19 \\
10 \mathrm{~F} \\
\mathrm{M}=29.2 \mathrm{y}\end{array}$ & $\begin{array}{l}\text { R CO, } 6 \text { conditions: } \\
\text { - } \text { black tea, } 0 / 100 \mathrm{mg} \mathrm{C} \\
\text { - } \text { coffee, } 0 / 100 \mathrm{mg} \mathrm{C} \\
\text { - } \text { hot water, } 0 / 100 \mathrm{mg} \mathrm{C}\end{array}$ \\
\hline
\end{tabular}

UMACL:

- HT improved by hot beverages vs no drink at $30 \mathrm{~min}$ $(\mathrm{p}=.0024)$ and $60 \mathrm{~min}(\mathrm{p}=.02)$ post drink; TA/EA unaffected

- EA and HT improved by caffeine at 30min (EA $\mathrm{p}=.0001$; HT $\mathrm{p}=.0025)$ and $60 \mathrm{~min}(\mathrm{EA} \mathrm{p}=.000$; HT $\mathrm{p}=.015)$ post drink; TA unaffected

STAI: improved by caffeine at $60 \mathrm{~min}$ post consumption $(\mathrm{p}=.05)$

LARS: Daylong - reduced sedation after caffeinated vs non-caffeinated beverages ( $\mathrm{p}=.033)$; Acute - caffeine level and time interaction $(\mathrm{p}=.025)$; reduced sedation by caffeinated vs non-caffeinated beverages, most prominent for coffee

STAI: no significant effects

\begin{tabular}{lll}
\hline $\begin{array}{l}\text { Hindmarch, } \\
2000\end{array}$ & $\begin{array}{l}\mathrm{N}=30 \\
15 \mathrm{~F}\end{array}$ & R CO, 5 conditions: \\
& $\mathrm{M}=27.3 \mathrm{y}$ & - black tea, $37.5 / 75 \mathrm{mg} \mathrm{C}$ \\
& & - hot water, $0 \mathrm{mg} \mathrm{C}$ \\
\hline Quinlan, & $\mathrm{N}=17$ & R CO, 6 conditions: \\
2000 & $9 \mathrm{~F}, 8 \mathrm{M}$ & - black tea, 37.5/75 mg C \\
Study 1 & $\mathrm{M}=35 \mathrm{y}$ & - coffee, $75 / 150 \mathrm{mg} \mathrm{C}$ \\
& & - hot water, 0 mg C \\
& & - no drink
\end{tabular}

LARS: No daylong effects but acute effects at drink 1 $(p=.0110)$ : reduced sedation after caffeinated beverages vs water $(p=.0049)$

UMACL: energetic arousal $(\mathrm{p}=.05)$, hedonic tone $(p=.007)$ increased by caffeinated vs noncaffeinated beverages; tense arousal rating unaffected

LARS: sedation reduced by caffeinated vs noncaffeinated beverages $(\mathrm{p}=.03)$; and coffee vs tea $(p=.04)$; dose-dependent: $150 \mathrm{mg}$ resulted in greater increase than $75 \mathrm{mg}(\mathrm{p}=.03)$

\begin{tabular}{lll}
\hline Quinlan, & $\mathrm{N}=15$ & R CO, 6 conditions: \\
2000 & $8 \mathrm{~F}, 7 \mathrm{M}$ & - black tea, 0/25/50/100 \\
Study 2 & $\mathrm{M}=33.9 \mathrm{y}$ & /200 mg C \\
& & - hot water, 0 mg C
\end{tabular}

UMACL: caffeine increased energetic arousal ( $p=.006)$; dose-dependent $(\mathrm{p}=.046)$ : lowest and highest dose produced largest increase; tense arousal, hedonic tone unaffected

LARS: caffeine ( $\mathrm{p}=.0003)$ reduced sedation; dosedependent $(\mathrm{p}=.0003)$, all doses except $50 \mathrm{mg}$

\begin{tabular}{cllc}
\hline De Bruin, & $\mathrm{N}=26$ & R PC CO, 2 conditions: & BL VAS: more alert $(\mathrm{p}<.001)$ and less calm $(\mathrm{p}=.008)$ \\
2011 & $20 \mathrm{~F}, 16 \mathrm{M}$ & - black tea & \\
aftudy 1 & $\mathrm{M}=30.7 \mathrm{y}$ & - placebo tea & \\
\hline De Bruin, & $\mathrm{N}=32$ & R PC CO, 2 conditions: & BL VAS: More alert after tea $(\mathrm{p}=.021)$ and tendency \\
2011 & $15 \mathrm{~F}, 17 \mathrm{M}$ & - black tea & towards greater feelings of contentment $(\mathrm{p}=.085)$ \\
Study 2 & $\mathrm{M}=30.3 \mathrm{y}$ & - placebo tea & after tea vs placebo; calmness rating unaffected \\
\hline
\end{tabular}

${ }^{1} R=$ Randomised, $C O=$ Crossover, $P C=$ Placebo controlled; ${ }^{2}$ LARS, Line Analogue Rating Scale; UMACL,

UWIST Mood Adjectives Checklist; HT = hedonic tone; TA = tense arousal; EA = energetic arousal; State

Trait Anxiety Inventory; BL VAS, Bond-Lader Visual Analogue Scale 


\section{Investigating the effects of tea, water and}

a positive affect induction on mood and creativity

Einöther, S.J.L., Baas, M., Rowson, M., \& Giesbrecht, T. (2015). Investigating the effects of tea, water and a positive affect induction on mood and creativity. Food Quality and Preference, 39, 56-61. 


\section{ABSTRACT}

Positive affect has been shown to be predictive of improved creativity. This study investigated the immediate effect of the tea consumption experience on positive affect and creativity, compared to both a neutral and positive control condition. Regular tea drinkers $(\mathrm{N}=150)$ were allocated to three conditions: 1$)$ tea preparation and consumption, 2$)$ cold water consumption, or 3) a positive affect induction. Participants completed the Affect Grid pre and post intervention, and measures of creativity and motivation post intervention. Tea consumption increased the valence dimension of mood, similar to the positive affect induction. Although it was expected that positive affect induction and tea consumption would improve creativity, we observed a trend in that direction on one measure of creativity (showing faster insights for difficult problems), but that effect did not reach statistical significance. Our study shows that a simple everyday activity such as tea consumption can effectively boost mood immediately after consumption. 


\section{INTRODUCTION}

Tea consumption is commonly associated with feelings of satisfaction (Desmet \& Schifferstein, 2008). Moreover, there are indications that tea consumption affects mood. According to the frequently used framework by Russell and colleagues (Russell, 1989; Russell \& Carroll, 1999) mood states can be reduced to the dimensions of valence and arousal. While valence refers to the degree to which an affective state is pleasurable (i.e., negative vs. positive), arousal denotes the degree of activation or energy (i.e., low vs. high).

Generally, research has focused on tea's arousing effects, due to the stimulant caffeine (Ruxton, 2008). Findings suggest effects can be separated into three phases (Quinlan et al., 1997; Aspen \& Quinlan, 1998b; Quinlan et al., 2000). The first phase, immediately after consumption, involves small and transient increases in heart rate and blood pressure, resulting in feelings of arousal, as well as more pronounced increases in skin conductance mainly due to sensory effects. The second phase, 10 to 30 minutes post-consumption, involves peak responses in skin temperature and skin conductance, likely due to temperature. In the last phase, 30 to 60 minutes post-consumption, tea ingredients caffeine and theanine have reached relevant plasma and brain concentrations (Magkos \& Kavouras, 2005; Van der Pijl et al., 2010), again resulting in increased (Hindmarch et al., 1998) and improved attention and alertness (De Bruin et al., 2011).

Regarding valence or pleasure, findings are limited. Two studies found improved hedonic tone 30 to 60 minutes after drinking a hot (caffeinated) beverage (Quinlan et al., 2000; Quinlan et al., 1997). Notably, theanine seems to improve relaxation and stress relief (Kimura et al., 2007; Yoto et al., 2012). Studies to date however largely neglected another interesting possibility: that positive affect may increase during and/or immediately after consumption, due to the consumption experience itself. This tea consumption experience includes the combination of the preparation, sensory properties (i.e. smell, taste, mouthfeel, appearance) before, during and after consumption, and potentially even by simply providing a break from other activities. Thus, the primary aim of this study was to assess the immediate mood benefits of tea consumption. 
Positive affective states have been shown to promote positive, favourable characteristics and build enduring personal and cognitive resources (Lyubomirsky, King, \& Diener, 2005; Fredrickson, 2001). Hence, it is of interest whether simple everyday activities, such as tea consumption, can generate small improvements in mood, as these may accumulate to greater benefits over time. Moreover, positive affect also has immediate beneficial effects on a range of motivational and cognitive processes (Fredrickson, 2001).

Isen and colleagues (2004) investigated the relationship between iced tea consumption, positive affect, and creativity. Albeit with a limited sample $(n=62 ; n=52)$, two experiments showed that tasting a familiar, branded iced tea (or receiving a bag of candies, a validated positive control) led to more creativity and greater positive affect than tasting an unfamiliar, unbranded iced tea and a control group that received nothing or water. Specifically, after consuming the iced tea (or receiving the candies), participants performed better on a creative problem solving task (an adapted Remote Associate Test; Mednick, 1962) and generated more unusual and pleasant first associates to a randomly selected letter of the alphabet.

It should be noted that these studies are somewhat restricted because of limited number of trials (i.e. 7 items of moderate difficulty). However, it does seem that consumption of a pleasant drink can increase creative problem solving to a similar extent as a well-known positive affect induction. Moreover, the study also showed the importance of the product being familiar and branded. The authors proposed that the mechanism by which iced tea consumption improved creativity, was through improved positive affect. Hence the secondary aim of the current study was to assess the effect of tea consumption on creativity.

Meta-analyses of mood-creativity research revealed that positive mood states lead to more creativity than neutral states (Baas, De Dreu \& Nijstad, 2008; Davis, 2009). Specifically, induction of positive mood, for example by a small gift, a short comedy film, or recalling a past happy event, exerts significant positive effects on creative problem solving (Estrada, Young, \& Isen, 1994; Isen, Daubman, \& Nowicki, 1987). Additionally, participants higher in positive affect solved more insight problems (Estrada et al., 1994; Isen et al., 1987), i.e. problems for which the correct solution ap- 
pears as a flash of insight, typically after non-conscious restructuring of the problem rather than deliberate and analytic processing (Sio \& Ormerond, 2009).

De Dreu, Baas, and Nijstad (2008) proposed a dual pathway model for the relation between mood and creativity. Creativity can be a function of either cognitive flexibility (i.e. broad attention, accessing multiple cognitive categories), or cognitive perseverance (i.e. focused attention, persevering few cognitive categories), and these pathways are moderated by mood. Mood states high in valence and arousal (i.e., happy) are associated with more creativity than neutral states via increasing cognitive flexibility, while moods high in valence but low in arousal (i.e. relaxed) are not (Baas et al., 2008; De Dreu, et al., 2008). Negative activating moods (i.e., anger, fear) decrease flexibility, but can increase creativity as compared to neutral ones through stimulating perseverance (Nijstad, De Dreu, Rietzschel, \& Baas, 2010), while negative deactivating moods (e.g., sadness) do not.

Moreover, recent studies attribute a perhaps even more important role to motivation, as activating mood states with a promotion focus (i.e., happiness) have been related to cognitive flexibility, while activating mood states with a prevention focus (i.e., fear) are related to persistence (Baas, De Dreu, \& Nijstad, 2011). Further evidence for the benefits of positive mood states for creativity comes from an fMRI study. Participants experiencing more positive affect showed different patterns of preparatory brain activity preceding each solved insight problem (Subramaniam, Kounios, Parrish, \& Jung-Beeman, 2009). Particularly, positive affect altered activity in the Anterior Cingulate Cortex (ACC) during response preparation. This activity is thought to be involved in executive control and is hypothesized to facilitate problem processing, i.e., fast and relatively automatic rather than a slow analytical process. Indeed, positive affect has been shown to lead to faster insights than negative affect (De Dreu et al., 2008).

The present study investigated the immediate effect of the tea experience on positive affect and creativity, compared to both a neutral and positive control. We hypothesized that tea consumption will increase positive affect, which in turn improves creative problem solving, as compared to a neutral control, and to a similar extent as a positive control. Tea was provided branded, following Isen et al. (2004), and involved preparation, while largely excluding the effects of ingredients (i.e., caffeine and theanine). 
The study used measures of affect, and divergent and convergent creativity. Divergent tasks are open-ended, designed to assess one's ability to generate original ideas or solutions. Convergent tasks are problem solving tasks, with a single correct answer that can be solved through methodical, analytic processing, or through sudden insight (Sio \& Ormerond, 2009). Finally, as positive mood can affect motivation, two measures of motivation were included. 


\section{METHODS}

\section{Participants}

One-hundred-fifty Dutch tea drinkers were recruited. Participants were predominantly female (77\%), with an average age of 34.48 years ( \pm SD 7.72 ) who drank black tea at least 5-6 times per week (69\% drank tea on a daily basis).

\section{Design}

Participants were assigned at random to three parallel conditions: 1. preparation and consumption of a cup of branded black tea (Lipton Yellow Label), 2. consumption of a glass of cold water (neutral control), 3. positive affect induction (positive control).

\section{Procedure}

Participants were tested individually in a lab setting. They were instructed how to use the mood test (i.e. Affect Grid), followed by short neutral film clip (3'26') to ensure a similar neutral affective state. In this clip, originally a screen saver, sticks of different colours fall on top of each other. Next, the first Affect Grid was administered and participants were assigned to one of three conditions.

In the tea condition, participants were provided with a cup of hot water, a branded tea bag, sugar, milk, and a teaspoon, and were instructed to prepare and drink the tea as preferred (e.g. strength, with or without milk and/ or sugar). This ensured that although experienced in a lab context, the tea consumption resembled closely how participants would generally prepare and drink their tea. This meant that participants were allowed to consume their tea exactly as they liked it. We assumed that this would allow us to achieve the maximal effect of tea on mood state. We did not expect glucose to affect outcomes, given that performance facilitation by glucose pertains to verbal memory (de Bruin \& Gilsenan, 2009) and mood effects have not been found at relevant doses (Sunram-Lea, Owen, Finnegan, \& Hu, 2011). 
In the positive affect condition, participants were asked to describe a recent event that made them feel very happy and cheerful. This allowed comparing effect size for tea consumption to a well-known and effective affect induction (Baas et al., 2008). In the water condition participants consumed a glass of cold water. Water has been previously used as a neutral control (Isen et al., 2004) and is a realistic everyday alternative to tea.

After consumption or the affect induction, the second grid was administered, followed by the creativity tasks, a motivation task and scales. The duration of the three conditions was kept constant at 10 minutes between the two Affect Grid administrations.

\section{Measures}

\section{Affect Grid}

The Affect Grid (Russell, 1989) is a two dimensional mood scale, assessing positive affect by the perceived degree of valence and arousal. Participants place a single mark in a 19x19 grid, defined by a y-axis describing arousal (extreme low to extreme high arousal) and an $\mathrm{x}$-axis describing valence (extreme low to extreme high pleasure). It allows for fast and repeated mood assessment, which is important as effects are expected to be subtle and short-lived.

\section{Remote Associates Task (RAT)}

The RAT (Mednick, 1962) consists of word triads (e.g. "manners, round, tennis") for which a single solution word relates to all three (e.g. table: table manners, round table, table tennis). A 30 item Dutch (computerised) version was used (easy, neutral and difficult items; each $n=10)$.

\section{Alien Creature Test}

This test assesses the ability to violate category boundaries in creative thinking (Ward, Patterson, \& Sifonis, 2004). Participants were asked to "Draw an alien creature, the way that you think an alien would look". The drawings were scored using a standardized 
methodology on six clearly defined characteristics: the less similar to known earth-like animals (e.g. more than two eyes in its head, asymmetrical, etcetera), the more original. This test is based on the rationale that, in general, people base their creations on known properties and that drawings that diverge from these properties and violate category boundaries are more creative.

\section{Unsolvable Anagrams}

Anagrams are words of which the letters have been shuffled to form non-words (e.g. "etrnce" = "center"). Participants are asked retrieve the word for four anagrams that increase in difficulty, with the fourth being unsolvable. Time spend on the unsolvable anagram indicates motivation and persistence (Friedman \& Elliott, 2008).

\section{Workload scale}

A 14 item Dutch version of the NASA TLX scale was employed measuring motivation, interest in the task, eagerness to perform well, irritation after the task, mental demand, physical and temporal demand, performance, effort and frustration (Dijkstra, 1999).

\section{Data Analysis}

Affect Grid scores were analysed via ANCOVA, with condition as between-subject factor and pre-condition scores as a covariate. Accuracy and reaction times on the RAT were analysed via generalised linear mixed models, with condition (3 levels: positive affect induction, tea consumption, water consumption) as between-subject factor, difficulty (3 levels: easy, neutral, difficult) as within-subject factor, and subject as a random effect.

Analysis of reaction time for correct responses only was complicated by the absence of correct responses for difficult items for some participants (24 out of 150 participants). The adjustment for these missing data involved the considerable but necessary assumption that reaction times to difficult tasks could be inferred from reaction times to easier tasks. Specifically, participant's reaction times on difficult tasks were estimated by their performance relative to the average on easy and neutral tasks. The analysis was conducted employing treatment, difficulty level and their interaction as fixed effects, 
and subject as a random effect. Assessment of the interaction in question was conducted by comparing Least Squared Means as generated by the GLIMMIX procedure in SAS v9. These LSMeans are predicted population margins, in that they estimate the marginal means over a balanced population.

Originality of drawings was analysed via a Kruskal-Wallis test and reaction times on the unsolvable anagram and the workload items were analysed via one-way ANOVA, condition as between-subject factor. Log transformation was applied if distributions suggested non-normality and a positive skew. There were ten missing observations for the drawings (due to failure to follow instructions) and one for the anagram, which were considered independent of condition and no corrective action was taken.

Hypothesis tests employed a $5 \%$ level of significance. Where a significant effect or interaction was identified, a Tukey-Kramer adjustment was applied to pairwise tests. 


\section{RESULTS}

\section{Mood}

There was a significant effect of condition on valence $(F(2,146)=10.96, p<0.01)$ and arousal $(F(2,146)=23.78, p<0.01)$ (Fig. 1). Water consumption provoked lower pleasantness than tea consumption $(p=0.01)$ or the positive affect induction $(p<0.01)$, while the difference between the latter two was not significant. Also, the positive affect induction provoked higher arousal than either tea or water consumption $(p<0.01$ each) and there was a non-significant suggestion that tea leads to lower arousal than water $(p=0.08)$.

\section{Creativity}

On the RAT, there was no effect of condition on overall accuracy or reaction times, either across difficulty levels or in interaction with difficulty level. A difficulty level effect (Accuracy: $F(2,147)=0.14, p<0.0001 ;$ Response Time: $F(2,294)=81.46$, $p<0.0001)$ reflected the best performance for the easy tasks. With regard to reaction times for correct responses only, there was a significant condition by difficulty interaction $(F(4,277.1)=2.53, p=0.04)$ (see Figure 2). For difficult tasks, the comparison was close to significant $(F(2,350.7)=2.72, p=0.07)$, with slower responses after water than either tea or the positive control (both $p$ 's $<0.05$ ). Again there was a significant effect of difficulty $(F(2,277.1)=81.99, p<0.01)$, with fastest responses for easy tasks. There were no significant effects on the alien drawings.

\section{Motivation and Workload}

There was no effect of condition on the anagram. There was an effect for workload item 6 (i.e., no desire for success; $F(2,134)=3.07, p=0.05$ ) reflecting that tea provoked a lower score, thus a greater desire for success than the positive control. Furthermore, an effect for item 10 (i.e. physical effort; $F(2,128)=3.63, p=0.02$ ) indicated that more physical effort was perceived to be required after positive control than after water consumption $(p=0.03)$. 


\section{DISCUSSION}

We hypothesized that preparation and consumption of tea would increase positive affect, which in turn may improve creativity. Results demonstrated that tea consumption improved the valence of mood, similar to a validated positive affect induction. Notably, the positive control also provoked higher arousal than tea or water consumption. Moreover, there was a trend that the tea consumption and positive control conditions led to faster responses on the RAT for difficult problems, one of the creativity outcomes, but effects fell shy of significance. There was no effect on accuracy of the RAT solutions or performance on the Alien Creatures Test. Finally, there were no changes in motivation resulting from the conditions.

The current study was the first to assess self-reported mood benefits immediately after tea consumption. In line with findings on satisfaction (Desmet \& Schifferstein, 2008), physiology (Aspen \& Quinlan, 1998; Quinlan et al., 2000), and consumer belief, we demonstrated that tea consumption improves valence or pleasure. Notably, the level of pleasure induced by tea consumption approached the level induced by the positive control. The positive control provoked higher arousal than tea or water consumption.

These findings are of importance not only to the research on the benefits of tea, but also to the research on inductions of positive affect and positive mood in general. With the ever-growing evidence on the role of positive emotions in short and long term well-being and success (e.g., Fredrickson, 2001; Lyubomirsky et al., 2005), it is viable that simple everyday activities generate small improvements in mood which may accumulate to greater benefits over time (c.f. Lyubomirsky et al., 2005). Our study shows that a simple everyday activity such as tea consumption can effectively boost mood for a short period of time after consumption.

This study was also the first to address effects of tea on creativity. It was hypothesized that the positive affect induced by tea consumption (and the positive affect induction) may improve creativity. However, only one measure, namely insight for difficult problems, showed a trend in that direction, but the effect did not reach statistical significance. Notably, the majority of studies addressing creative problem solving focused on providing correct solutions rather than response speed. Positive affect inductions gener- 
ally increase the number of solved items of moderate difficulty as compared to no intervention or a physical activity intervention (Isen et al., 1987; Estrada et al., 1994). Finally, Isen et al. (2004) also showed that different positive affect inductions, including the iced tea consumption, resulted in solving more items of moderate difficulty, but unfortunately effects of the different inductions were not reported separately.

Few studies also included the time needed to reach a (correct) solution. Positive mood is known to broaden the attentive state and therefore the detection of semantic associations (Rowe, Hirsch, \& Anderson, 2007), resulting in faster solutions as compared negative mood (De Dreu et al., 2008). Moreover, participants also solved problems faster with insight than they did analytically (Subramaniam et al., 2009). Thus, being faster in identifying the correct solution could indicate positive affect may lead to faster insights, indicative of a more associative and flexible processing style (De Dreu et al., 2008). Alternatively, positive mood may stimulate the use of more simple decision heuristics. However, this would lead to more incorrect responses, which was not the case as the number of correctly solved items was not affected by condition. Thus, while this trend of faster insight for difficult problems must be interpreted with caution, it is very interesting to explore in future studies, for example using a larger set of difficult items.

The strength of this study is the use of scientifically validated measures of mood and creativity, with a procedure that is close to a regular tea consumption experience. Furthermore, the positive control enables the comparison between tea and a known mood enhancer, and across studies on affect and creativity. Notably, the positive control also increased arousal. Valence and arousal, albeit mediated by separate neural systems, are nevertheless often highly correlated (Ashby, Isen, \& Turken, 1999). Hence, it comes as no surprise that the positive control also increased arousal. Tea consumption positively affected valence but not arousal. Yet, in contrast to the need for both high arousal and high valence to induce creativity identified in the meta-analysis by Baas et al. (2008), there was no difference in creative problem solving between the positive control and the tea condition.

A limitation is that it remains to be disentangled which aspect(s) of tea consumption led to changes in valence of mood. Firstly, these findings may be attributed to the fact that the tea was warm. Experiencing physical warmth has been shown to affect 
social information processing, in that holding a warm drink increased perceived warmness of another person (Williams \& Bargh, 2008) and perceived emotional intimacy (Ijzerman \& Semin, 2009). While a cold tea-based drink improved positive affect as indicated by improved creativity (Isen et al., 2004), the warm temperature may provide an additive effect on mood. Also, although tests were administered immediately after ingestion thus leaving no time for actual ingredient effects, anticipatory effects of ingredients on performance are known to occur. For example, exercise performance can be improved by mere mouth rinsing, without ingestion, with a drink containing carbohydrates (Chambers, Bridge, \& Jones, 2009) or caffeine (Beaven, Maulder, Pooley, Kilduff, \& Cook, 2013). It is hypothesized that these ingredients may exhibit direct effects on reward centres (ACC and striatum) via the oral receptors (Chambers et al., 2009). Moreover, the expectation of having consumed caffeine can improve mood (Ijzerman \& Semin, 2009), as well as performance (Dawkins, Shahzad, Ahmed, \& Edmonds, 2011). Furthermore, the tea condition also differed by included tea preparation, which may have stimulated personal rituals that can affect mood. Moreover, participants needed more time to drink the tea. Finally, although the current study strived to make the tea consumption experience as realistic as possible by allowing preparation as preferred, the study was conducted in a laboratory setting. Moreover, as participants were randomly assigned to be tested between 9 am and 9 pm, testing did not necessarily coincide with their habitual tea consumption time. One may hypothesize that effects may be bigger when testing coincides with normal intake time. Hence, the current study might underestimate actual valence effects.

To conclude, findings extend earlier work by confirming the supposed immediate effects of tea consumption on positive affect. Specifically, we demonstrated that tea consumption positively affects valence of mood, as does a positive affect induction, while water does not. There was a non-significant trend that tea consumption and the positive control resulted in faster insights for difficult problems, while leaving the number of correct solutions or originality of drawings unaffected. Although the exact mechanism needs to be further disentangled, the findings indicate that tea consumption can improve mood. 


\section{FIGURES}

Fig. 1 Affect Grid mean valence and arousal scores post treattment

(error bars indicate SD's; the score 10 reflects the neutral middle)

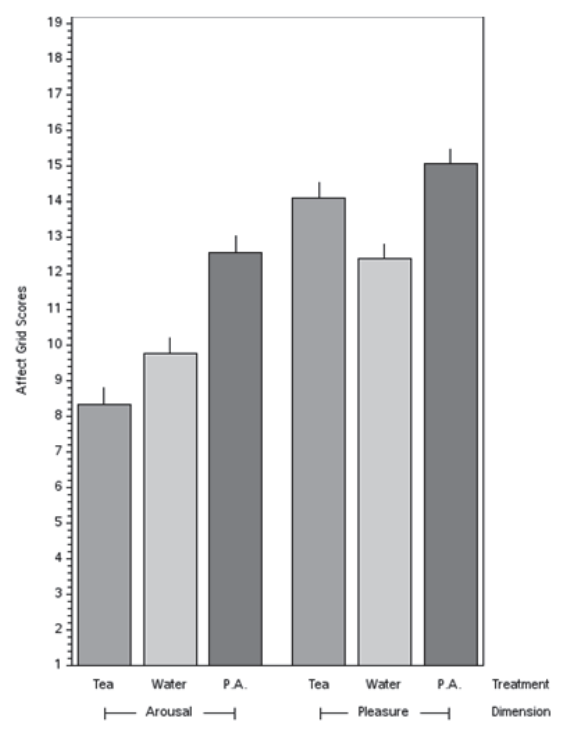

Fig. 2 RAT mean response times for correct responses, total and per difficulty level (error bars indicate SD's)

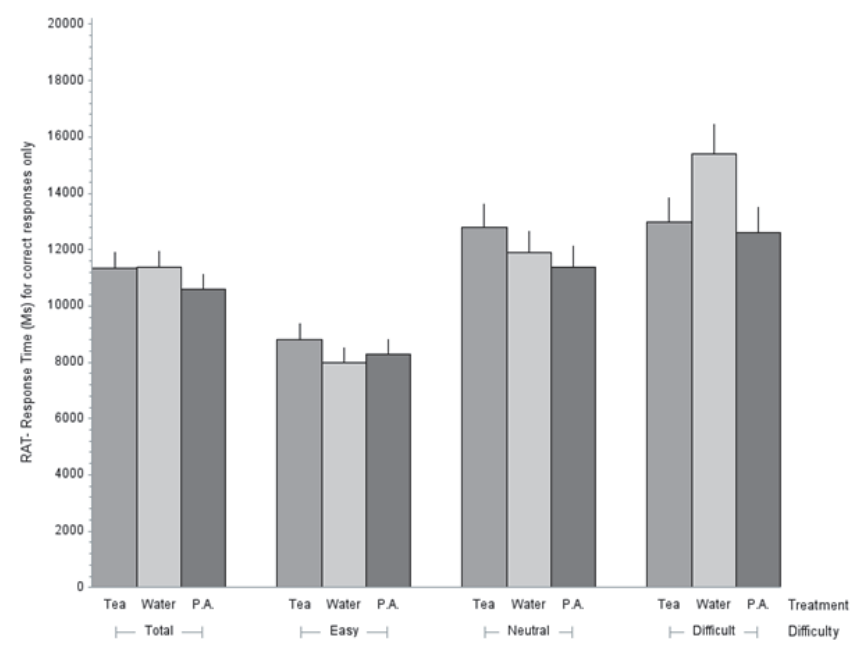





\section{Infusing Pleasure:}

Mood effects of the consumption of a single cup of tea

Einöther, S.J.L., Rowson, M., Ramaekers, J.G., \& Giesbrecht, T. (Submitted). Infusing pleasure: Mood effects of the consumption of a single cup of tea. 


\section{ABSTRACT}

Tea has historically been associated with mood benefits. Nevertheless, few studies have empirically investigated mood changes after tea consumption. We explored immediate effects of a single cup of tea up to an hour post-consumption on self-reported valence, arousal, discrete emotions, and implicit measures of mood. In a parallel group design, 153 participants received a cup of tea, water or placebo tea. Immediately after consumption, tea increased valence but reduced arousal, as compared to placebo. There were no differences at later time points. Discrete emotions did not differ significantly between conditions, immediately or over time. Water consumption increased implicit positivity as compared to placebo, with no effect on implicit negativity. Finally, consumption of tea and water resulted in higher interest in activities overall and in specific activity types compared to placebo. The present study shows that effects of a single cup of tea may be limited to an immediate increase in pleasure and decrease in arousal, which can increase interest in activities. More servings over a longer time may be required to evoke arousing effects and appropriate consumption settings may evoke more enduring valence effects. 


\section{INTRODUCTION}

Many people report to eat, drink, or smoke to feel good (Bennett, 1988). That some of the products which we consume as part of our diet can affect mood is conceivable. For instance tea, the most consumed beverage in the world after water, has long been associated with mental benefits, such as relaxation and clarity of mind (Graham, 1992; Shimbo, Nakamura, Jing, Kizuki, Seino et al., 2005). While these alleged benefits enjoy strong support from consumers, scientific evidence has been largely anecdotal until recently.

Based on the scientific literature to date (Einöther \& Martens, 2013), acute mental effects of tea can be characterized along two dimensions (i.e., cognitive and affective) and three post-consumption stages (i.e., immediately after, shortly after, and after 30 minutes or more). Cognitive effects are well established in terms of improved attention after 30 minutes (De Bruin, Rowson, Van Buren, Rycroft, \& Owen, 2011), and are ascribed to tea ingredients theanine and caffeine. Yet, little is known about effects of tea on affective states, in particular 'emotions', which are relatively brief but intense (Mesquita \& Frijda, 1992), in comparison to 'mood', a low-intense but enduring state (Lucas, Diener, \& Larsen, 2009).

Studies to date typically focused on effects experienced 30 or more minutes after tea consumption, and consistently reported increased self-reported alertness and arousal, in line with tea's attention benefits (De Bruin et al., 2011; Hindmarch, Quinlan, Moore, \& Parkin, 1998; Quinlan, Lane, Moore, Aspen, Rycroft et al., 2000; Quinlan, Lane, \& Aspinall, 1997). Quinlan et al. (1997) also reported improved hedonic tone after tea, compared to water, but Quinlan et al. (2000) showed no effect on contentment. Importantly, in one study the tea contained more caffeine, $100 \mathrm{mg}$ compared to the normal 35-61 mg (Quinlan et al., 1997) and in others participants consumed multiple cups (De Bruin et al., 2011; Hindmarch et al., 1998; Quinlan et al., 2000).

Mood may also be affected by the "tea experience" itself. During a typical tea consumption moment, this potentially includes the effect of preparation rituals (Dohle, Rall, \& Siegrist, 2014), expectations regarding consumption or effects thereof (e.g. regarding 
effects of caffeine; Dawkins, Shahzad, Ahmed, \& Edmonds, 2011), anticipation of consumption pleasure (Rozin, 1999), and providing a break from other activities (e.g. brief work withdrawal; Miner, Glomb, \& Hulin. 2005). Importantly, it also includes the perception of its sensory properties (i.e., smell, taste, mouth feel, and appearance) before, during and after consumption. Tea consumption has been shown to exert physiological effects due to sensory properties (immediate: small increases in heart rate and larger increases in skin conductance), and due to its temperature (5 - 30 minutes postconsumption: increased skin temperature and skin conductance) (Aspen \& Quinlan, 1998; Quinlan et al., 1997; Quinlan et al., 2000). This opens up a range of opportunities for investigating how tea consumption may influence affective state.

One study to date partially addressed this hypothesis (Einöther, Baas, Rowson, \& Giesbrecht, 2015). Tea preparation and consumption improved valence of mood immediately after consumption compared to water. Improvements were comparable to those produced by positive imagery. It this seems that simple everyday activities such as tea preparation and consumption can improve mood. Moreover, growing evidence shows that small improvements in mood may accumulate to greater benefits on well-being over time (e.g., Fredrickson, 2001; Lyubomirsky, King, \& Diener, 2005). Also, positive affect has been shown to improve cognitive processes such as global information processing (Clore \& Huntsinger, 2007) and creativity (Baas, De Dreu, \& Nijstad, 2008). Thus, it may be that tea consumption also affects other cognitive processes via improved mood. Einöther et al. (2015) reported that positive affect from tea consumption tended to decrease response time in creativity tests.

In sum, data on affective qualities of tea consumption are mixed. Notably, while some scientists assume that affective states can be reduced to two dimensions (Russell \& Carroll, 1999; Watson, Clark, \& Tellegen, 1988), others propose a number of discrete emotions, i.e. happiness, fear, and sadness, each representing unique experiential states with a distinct cause (Ekman, 1992). As such, affect questionnaires are broadly classified as bipolar or discrete. Bipolar questionnaires such as the Affect Grid (Russell, 1989) assess valence, the degree of pleasure (i.e., negative/positive), and arousal, the degree of activation (i.e., low/high), while others assess discrete emotions, e.g. Profile of Mood States (McNair, Lorr, \& Droppleman, 1992). 
Discrete emotions have been related to food and beverage consumption, particularly to sensory attributes. For example, sweet taste has been associated with happiness and surprise, and bitter taste with anger and disgust (Rousmans, Robin, Dittmar, \& Vernet-Maury, 2000). Also, discrete emotion based tools such as PrEmo® and EsSense Profile ${ }^{\circledR}$ have been able to distinguish between products from the same category (i.e. breakfast drinks or salty snacks) with different sensory attributes (Gutjar, de Graaf, Kooijman, de Wijk, Nys et al., 2014; King, Meiselman, \& Carr, 2010).

The primary aim of this study was to assess effects of tea consumption on valence and arousal dimensions of mood (as measured by the Affect Grid), immediately postconsumption. In line with findings from Einother et al. (2015), we hypothesised that consumption of a cup of tea would increase the valence dimension of mood immediately after consumption, as compared to water and placebo tea (hypothesis 1). The secondary aim was to assess effects of tea consumption on the valence and arousal dimensions of mood over time, up to 60 minutes after consumption. Based on findings by De Bruin et al. (2011), we hypothesised that consumption of a cup of tea will increase arousal of mood over time, and particularly at 60 minutes post consumption, as compared to water and the placebo tea (hypothesis 2).

In addition, we investigated the effects of tea consumption on self-reported discrete emotions, immediately and up to 60 minutes post-consumption, using the Affect Self-Report scale (ASR). As this was the first study addressing discrete emotions in relation to tea consumption, this outcome was considered exploratory. Notably, the ASR also includes dimensional aspects of mood, which are similar to the ones of the Affect Grid. However, to our knowledge, this scale has not been used to capture effects of food and beverages consumption before. Therefore, we considered its outcomes as secondary to the Affect Grid, with regard to tapping into the valence and arousal dimension of mood.

Although validity of self-report mood measures has been demonstrated, it should be noted that mood changes can occur in the absence of conscious experience required by self-report (Larsen, Berntson, Poehlmann, Ito, \& Cacioppo, 2008; Winkielman \& Berridge, 2004). Since affect can influence how we think and act, i.e., affect-asinformation theory (Clore \& Huntsinger, 2007) or broaden-and-build theory of positive 
emotion (Fredrickson, 2004), measures of behavioural consequences may provide a more complete picture of affective state. We explored immediate effects on two implicit mood tests: the Implicit Positive and Negative Affect Test (IPANAT) and the Momentary Pleasant Activity List (MPAL). Both outcomes were considered exploratory, and whilst accompanied by hypothesis tests for completeness, the outcomes of these tests are for hypothesis-building purposes only. 


\section{METHODS}

\section{Participants}

One-hundred-fifty-three Dutch regular tea drinkers (30 males; $M=33.5$ yrs \pm 8.33 ) were recruited. Volunteers were included if they consumed black tea without milk and sugar at least once a week, were 18-45 years of age, Dutch native speakers and nondyslexic (because of the linguistic nature of the tests). The study was conducted in compliance with the Declaration of Helsinki, and the protocol was approved by the Unilever Independent Ethics Committee at Wageningen University, The Netherlands. Participants signed an informed consent and were paid for participation.

\section{Design}

The study employed three parallel consumption conditions: $200 \mathrm{~mL}$ of black tea (Lipton Yellow Label), placebo tea (flavoured and coloured water), both served at a drinkable temperature of $60^{\circ}$ (Brown \& Diller, 2008), and water at room temperature. Tea was consumed without sugar and milk. The placebo tea looked and tasted like tea, but contained no real tea (ingredients: 93.4\% caramel coloured maltodextrin, $6 \%$ tea flavor and $0.6 \%$ silicon dioxide). Water, as the neutral control, is a realistic everyday alternative to tea. The experimenter who interacted with the subjects was not involved in the drinks preparation and was partially blinded to the drinks being consumed (i.e., with respect to the true tea and the placebo tea). Participants were randomly allocated to groups, resulting in 54 participants in the placebo group, 52 in the tea group and 47 in the water group.

\section{Procedure}

Participants attended the test location for a single session with up to six participants per session. Sessions lasted 75 minutes and were conducted between 10.00 and 22.00 hours, with time of testing counterbalanced across groups. Participants were told that the study aimed to investigate how people experience different types of drinks, and recruitment 
included questions regarding different drinks (e.g. coffee and cola), to ensure that they were unaware of the focus on tea.

Participants sat in individual booths to prevent them from seeing each other, with a personal computer and keyboard. After receiving general instructions and completing the informed consent form, participants were instructed on using the Affect Grid and viewed a short neutral film clip (3'26') to ensure a similar neutral affective state (Einöther et al., 2015). Before beverage consumption, baseline assessments of Affect Grid, ASR and IPANAT (but not MPAL) were completed. Next, participants were given one of the three beverages (tea, water, or placebo tea) and were allowed five minutes to consume their beverage. Subsequently, tests were administered at specified times up to one hour post-consumption (Table 1).

\section{Outcome measures}

Outcome measures are described below in the order of inclusion. A fifth unrelated measure, completed at baseline, and the 5, 30 and 60 minutes assessments, is not reported here.

\section{Affect Grid}

Valence and Arousal of mood were assessed with the Affect Grid (Russell, 1989) at baseline, immediately after consumption, 20 minutes after and every 10 minutes up to 60 minutes after consumption (7 times). Participants marked how they felt on a 19x19 grid with valence on the horizontal axis, ranging from unpleasant to pleasant, and arousal on the vertical axis, ranging from sleepy to active.

\section{Affect Self Report (ASR) scale}

The Affect Self Report scale (Christie \& Friedman, 2004) assesses dimensional emotions (i.e., valence or arousal) as well as discrete emotions (e.g. happiness, anger, content etc.). It has been used to link self-reported emotions to autonomic responses (Stephens, Christie, \& Friedman, 2010). Our version included the original 10 dimen- 
sional items, 10 discrete items and four additional positive items (interested, joyful, optimistic, and curious). The ASR was completed four times; at baseline, immediately after consumption, 30 minutes and 60 minutes after consumption.

\section{Implicit Positive and Negative Affect Test (IPANAT)}

This implicit mood test (Quirin, Kazen, Rohrmann, \& Kuhl, 2009) is based on the framework of positive and negative affect (Watson et al., 1988). Participants rated to what extent artificial words (e.g. SAFME) fit with three positive (happy, cheerful, energetic) and three negative (helpless, tense, inhibited) emotions. Implicit positivity is the average fit with the positive emotion words, and implicit negativity is the average fit with the negative emotion words. As a state measure, implicit positivity was significantly related to self-reported positive affect (PA) and negativity to self-reported negative affect (NA), while positivity was not related to NA or negativity to PA (Quirin et al., 2009). Due to the nature of the task, it was only completed twice; at baseline, and immediately after consumption.

\section{Momentary Pleasant Activities List (MPAL)}

The Pleasant Activities List (Roozen, Wiersema, Strietman, Feij, Lewinsohn et al., 2008) assessed the frequency and enjoyability of common activities in the past 30 days. In this adapted version, participants rated 50 of the original activities on how pleasurable it would be to perform them, at this moment. Scores were averaged to an overall interest score and scores per activity type, i.e. Social Activities (SA), Sensation Seeking Activities (SSA), Domestic Activities (DA), Culture, Science and Traveling (CST), Passive, Relaxing Activities (PRA), Sport-Related Activities (SRA), and Intimacy and Personal Attention (IPA). Due to the nature of the task, the PAL was administered only once, immediately after consumption. 


\section{Data Analysis}

Immediate Affect Grid valence and arousal scores were assessed via ANCOVA, with condition as fixed effect and baseline as covariate (hypothesis 1). The total Area Under the Curve (AUC), derived from Affect Grid assessments at baseline, 5, 20, 30, 40, 50 and 60 minutes after consumption, was assessed similarly via ANCOVA (hypothesis 2). Where an effect of condition was identified, a Tukey-Kramer adjustment was applied to all pairwise comparisons. In a few instances, but never at baseline, duplicate or triplicate scores were recorded due to a fault in the computerised program. Here the approach was as follows: where the range of scores was less than three units, the potential for error was considered negligible and the average was taken, but where the range of scores exceeded three units, the data was omitted from the AUC analysis. Consequently, three AUC scores were averaged, i.e. one for arousal (Subject 24, T=5) and two for valence (Subject 130, $\mathrm{T}=30$ and $\mathrm{T}=60$ ), and three were excluded, i.e. one for arousal (Subject 69, $\mathrm{T}=60$ ), and two for valence (Subject 100, $\mathrm{T}=30$; Subject 103, $\mathrm{T}=60$ ).

The ASR scale yielded two dimensional outcomes based on aggregated scores (arousal and valence), and ten discrete outcomes, based on individual scores. Dimensional outcomes were analysed in the same way as the Affect Grid dimensions. As discrete outcome residuals were not normally distributed (i.e. a lot of very low scores), the data did not lend itself to linear modelling and were analysed via a Kruskal-Wallis nonparametric test (immediate change from baseline, and AUC). IPANAT Positivity and Negativity scores were analysed via ANCOVA. The seven composite scores and the total score for the MPAL were analysed by a series of one-way ANOVAs, with a Tukey-Kramer adjustment applied to pairwise comparisons. 


\section{RESULTS}

\section{Affect Grid}

Table 2 shows the Affect Grid outcomes. Both immediate Valence $(\mathrm{F}(2,152)=3.53$, $\mathrm{p}=0.03)$ and Arousal $(\mathrm{F}(2,152)=4.31, \mathrm{p}=0.02)$ differed between conditions (See Fig. 1). Specifically, those who consumed tea displayed higher pleasure scores $(p=0.03$; based on post-hoc Tukey-Kramer-adjusted test) but lower arousal scores $(\mathrm{p}=0.02$; based on post-hoc Tukey-Kramer-adjusted test) than those who consumed placebo. Moreover, a difference significant only at the $10 \%$ level $(\mathrm{p}=0.07)$ between water and tea indicated lower arousal after tea as compared to after water consumption. There were no significant differences in Arousal or Valence over time (i.e. total AUC).

\section{ASR}

Table 2 also shows the means and standard deviations for the valence and arousal dimensions of the ASR. There was no evidence of a group effect on the ASR; either on the dimensional scales Arousal and Valence), or the discrete items (e.g., happiness, anger, sadness, disgust), immediately after consumption or over time (AUC).

\section{IPANAT}

Implicit positivity differed significantly between conditions $(F(2,152)=3.36, p=0.04)$, with the water evoking higher scores than placebo $(p=0.03)$. Differences in negativity were not significant (Table 3).

\section{MPAL}

The majority of factors in the Momentary Pleasant Activities List (MPAL) displayed good reliability (Cronbach $\alpha$ : SA $=0.837 ; \mathrm{SRA}=0.815 ; \mathrm{CA}=0.724 ; \mathrm{SSA}=0.719 ; \mathrm{CST}$ $=0.704)$. Two factors displayed acceptable reliability $(\mathrm{DA}=0.637$; PRA $=0.666)$ and only one displayed poor reliability $(\mathrm{IPA}=0.574)$. Figure 2 shows mean scores per factor. 
The Total Score showed an effect of condition $(F(2,152)=3.84, p=0.02)$, with higher scores after consumption of tea and water, compared to placebo (both $\mathrm{p}=0.05$ ). Conditions also differed with regard to Social Activities $(\mathrm{F}(2,152)=4.21, \mathrm{p}=0.02)$, with higher interest after water compared to placebo $(\mathrm{p}=0.02)$. Interest in Creative $(\mathrm{F}(2,152)$ $=4.01, \mathrm{p}=0.02)$ and Domestic Activities $(\mathrm{F}(2,152)=3.21, \mathrm{p}=0.04)$, was higher after tea compared to placebo (CA: $\mathrm{p}=0.02$; DA: $\mathrm{p}=0.05$ ), while interest in Culture, Science \& Travel $(F(2,152)=3.06, p=0.05)$ was higher after water compared to placebo $(p=0.05)$. There was no evidence of differences for Intimacy/Personal Attention, Sensation Seeking, Passive/Relaxing or Sports Activities. 


\section{DISCUSSION}

Whilst tea has historically been associated with mental benefits, few studies have empirically investigated these alleged effects. The current study explored the effects of a single cup of black tea, immediately after consumption as well as over time, on selfreported valence and arousal, discrete emotions and implicit mood, compared to cold water and a placebo tea. Specifically, we hypothesised that consumption of a cup of tea would increase valence of mood on the Affect Grid immediately after consumption (hypothesis 1), and increase arousal of mood on the Affect Grid over time (hypothesis 2), as compared to water and placebo tea. Measures assessing discrete emotions (ASR), implicit mood (IPANAT) and interest in activities (MPAL) were considered exploratory.

Hypothesis 1 was only partially confirmed. Immediately after consumption of tea, the valence aspect of mood was significantly increased on the Affect Grid, as compared to placebo, but not as compared to water. There were no differences over time between the different conditions and hypothesis 2 was therefore rejected. The ASR scale revealed no differences in relation to dimensions of emotions or discrete emotions. With regard to implicit mood, water consumption increased implicit positivity as compared to placebo, and tea and water consumption increased interest in activities overall and specific types of activities, compared to placebo.

Tea consumption resulted in an immediate yet subtle increase in valence of mood on the Affect Grid, as compared to placebo. These findings indicate that the consumption of a cup of tea increased the experienced pleasure. In contrast to Einöther et al. (2015), the difference between tea and water, albeit in the same direction, was not significant. As our tea was standardized (i.e. prepared by the staff), this may point to the importance of self-preparation. Indeed, simple preparation rituals like stirring or breaking can heighten product involvement and thereby pleasure experienced (Dohle et al., 2014; Vohs, Wang, Gino, \& Norton, 2013). Others have stressed the importance of anticipatory pleasure (Rozin, 1999), and the clearly distinguishable neural correlates of wanting, i.e. desire, and liking, i.e. the hedonic impact of the desired (Berridge \& Robinson, 2003). Also, 
our tea was unbranded, whereas branded products may elicit stronger positive emotions (Isen, Labroo, \& Durlach, 2004; Spinelli, Masi, Zoboli, Prescott, \& Monteleane, 2015). Interestingly, consumption of a single cup of tea reduced arousal immediately after consumption compared to the placebo tea and, albeit to a lesser degree, also compared to water. Notably, Einother et al. (2015) also demonstrated that tea (and to some extent water consumption) resulted in lower arousal than the positive affect induction. These findings may indicate a role for tea consumption in relaxation.

Neither valence nor arousal were affected beyond the initial measurement point, and hence were not demonstrably modulated over time by tea consumption. The former is perhaps not surprising considering earlier inconsistent findings (De Bruin et al., 2011; Quinlan et al., 2000), but the latter is. In line with caffeine's arousing effects, consumption of caffeinated beverages including tea, increased arousal after 30 to 90 minutes (Quinlan et al., 2000; De Bruin et al., 2011). Notably, in these studies participants consumed slightly larger volumes (300 and $400 \mathrm{~mL}$, respectively), or multiple cups of tea. Thus, a single $200 \mathrm{~mL}$ serving may not be sufficient for evoking arousing effects.

Markedly, the current study employed three measures to address valence of mood immediately after consumption, i.e. Affect Grid, ASR, and IPANAT. Yet only the Affect Grid, also the first measure employed here, demonstrated a difference in valence between drinks consumed. Neither the ASR, nor the IPANAT have been used before to measure effects of food and beverages consumption and may not be suitable for this purpose. Alternatively, findings may indicate that the effects of the tea consumption experience are rather transient and are only noticeable immediately after consumption. Indeed, effects were apparent immediate after consumption, but not over time.

Discrete emotions did not differ significantly between conditions. Potentially, effects of a single serving are too transient and subtle to be picked up by the ASR, as compared to more generic feelings of valance, and arousal, as assessed by the Affect Grid. Measuring distinct emotions could be of greater relevance to the social and situational consumption context in everyday life, e.g. catching up with a friend over tea or taking a break from work. 
Other exploratory measures revealed few differences between conditions. Firstly, water consumption increased implicit positivity as compared to placebo. Secondly, tea (and to some extent water) consumption resulted in a higher interest in activities overall, and specifically in creative and domestic activities (after tea), and social activities, culture, science and travel (after water). In line with the idea that people rely on affective states to inform their judgments (Schwarz, 1990), positive mood has been shown to generate interest in engaging in activities (Cunningham, 1988b), increase motivation for (moderately) enjoyable, but not dull, tasks (Kraiger, Billing, \& Isen, 1989), and foster prosocial behaviour such as initiating conversation (Cunningham, 1988a), and helping behaviour (Isen, 1999). Moreover, this increased interest in creative activities is thought-provoking in the light of faster creative problem solving following tea (Einöther et al., 2015). While the authors propose positive affect may lead to faster insights because of more flexible processing (De Dreu et al., 2008), current findings may propose a mechanism via greater interest in such a task.

Interestingly, in addition to differences between tea and placebo tea, we also demonstrated unexpected differences between water and the placebo, but less clear-cut differences between tea and cold water, compared to the previous study (Einother et al., 2013). In addition to the points discussed above (the preparation of the tea, and the fact that the tea was unbranded), a number of explanations may be provided.

Although the placebo tea has been developed to match the colour and appearance of a black tea and to resemble its taste, this finding may demonstrate that it was less liked, as compared to the water (and the tea) condition, or that there was somehow a mismatch between expectations and reality concerning the placebo. Previous studies using a similar placebo tea focused on alertness/ arousal over time rather than valence or pleasure immediately after consumption and therefore have not picked up this difference.

Moreover, there are some indications that (cold) water consumption can improve mood. Two studies found an immediate effect on alertness, which did not seem to be sustained over time (Rogers, Kainth, and Smit, 2001; Pross, Demazieres, Girard, Barnouin, Santoro, et al., 2013) while a third study did not (Edmonds et al, 2013). Hydration status and habitual consumption may play a role in this effect, as a relationship 
between dehydration and negative mood states has been confirmed (Benton \& Young, 2015). No studies to date however reported on valence effects of water. It may be the case that the refreshing nature of water does improve affective state, but it is unclear whether this effect is specific to water (and not for example the placebo) and why.

The current study adds to the growing understanding of the alleged mood benefits of tea consumption (Einöther et al., 2013; Einöther et al., 2015), by exploring effects of a single cup of tea on validated mood scales, as well as novel implicit tools. A limitation was the lab-like setting of the experiment; although of good reliability, generalizability can be questioned, as frequency and intensity of positive emotions to food are generally higher in appropriate contexts (Piqueras-Fiszman \& Jaeger, 2015). Another limitation was that the multiple measures and comparisons employed may have led to chance findings. A final limitation was that the drinks were not completely blinded and may have evoked different expectations. The placebo tea will have given rise to the expectation that one would be consuming tea and it seems this these expectations have not been met in the actual consumption. Previous studies comparing different drinks have indeed shown the importance of expectations (Hindmarch et al., 1998).

Investigating the tea consumption experience may require a holistic approach, including situational, emotional, physical and/or social contexts (Macht, Meiniger, \& Roth, 2005) as each triggers specific needs, expectations and attentional focus. Here, neither was included: participants were brought in a relatively neutral mood state before consumption, were not allowed to have social contact with others in their session, and were randomly tested between 9 am and $9 \mathrm{pm}$. As such, results might underestimate actual effects.

One study to date assessed effects of beverage consumption on mood and performance during ten work days (Bryan, Tuckey, Einother, Garczarek, Garrick et al., 2012). Participants who consumed more tea reported feeling less tired and performing better. However, mood reports were completed four times a day, not necessarily before or after beverage consumption. This set-up not only limits conclusions on causality, it also potentially underestimates effects of tea consumption on mood. Future studies could benefit from frequent but simple measures of positive affect in a naturalistic set- 
ting, potentially using consumer-oriented devices allowing us to monitor psychological as well as physiological outcomes, at home and work.

To conclude, tea consumption can boost valence of mood and reduce arousal immediately after consumption, and can increase interest in activities. Findings encourage further investigation into a number of areas. It seems that rather than focusing on effects of a single cup it would be advisable to further explore the immediate consumption moment, including preparation of the tea and the consumption context or need state, and the effects of multiple cups, perhaps even throughout the day. Furthermore, as there were some indications of increased interest in activities, it would be interesting to further investigate whether tea consumption also affects motivation, for example at work. 


\section{TABLES AND FIGURES}

Table $1 \quad$ Study procedure outline

\begin{tabular}{|c|c|c|c|c|c|c|c|c|c|}
\hline \multicolumn{2}{|c|}{ Outcome meaaure } & -10 & Drink & 5 & 20 & 30 & 40 & 50 & 60 \\
\hline \multirow[t]{2}{*}{ Self-report } & Affect Grid & $\mathrm{X}$ & & $\mathrm{X}$ & $\mathrm{X}$ & $\mathrm{X}$ & $\mathrm{X}$ & $\mathrm{X}$ & $\mathrm{X}$ \\
\hline & ASR & $\mathrm{X}$ & & $\mathrm{X}$ & & $\mathrm{X}$ & & & $\mathrm{X}$ \\
\hline \multirow[t]{2}{*}{ Implicit } & IPANAT & $\mathrm{X}$ & & $\mathrm{X}$ & & & & & \\
\hline & MPAL & & & $\mathrm{X}$ & & & & & \\
\hline
\end{tabular}

\section{Table 2 Affect Grid and ASR}

\begin{tabular}{|c|c|c|c|c|c|}
\hline \multirow{2}{*}{ Time } & \multirow{2}{*}{ Condition } & \multicolumn{2}{|c|}{ Arousal } & \multicolumn{2}{|c|}{ Pleasure } \\
\hline & & $\mathbf{M}$ & $95 \% \mathrm{CI}$ & $\mathbf{M}$ & $95 \% \mathrm{CI}$ \\
\hline \multicolumn{6}{|c|}{ Affect Grid } \\
\hline \multirow{3}{*}{$5 *$} & Placebo & 9.01 & $+/-0.86$ & 11.81 & $+/-0.86$ \\
\hline & Water & 8.76 & $+/-0.93$ & 12.36 & $+/-0.92$ \\
\hline & Tea & 7.30 & $+/-0.88$ & 13.44 & $+/-0.88$ \\
\hline \multirow{3}{*}{$A U C * *$} & Placebo & 8.51 & $+/-0.75$ & 12.19 & $+/-0.66$ \\
\hline & Water & 7.68 & $+/-0.80$ & 11.89 & $+/-0.71$ \\
\hline & Tea & 7.36 & $+/-0.76$ & 12.43 & $+/-0.67$ \\
\hline \multicolumn{6}{|c|}{ ASR } \\
\hline \multirow{3}{*}{5} & Placebo & -1.73 & $+/-0.34$ & 2.72 & $+/-0.43$ \\
\hline & Water & -1.89 & $+/-0.36$ & 3.42 & $+/-0.46$ \\
\hline & Tea & -2.16 & $+/-0.34$ & 3.01 & $+/-0.44$ \\
\hline \multirow{3}{*}{$A C^{* *}$} & Placebo & -1.72 & $+/-0.28$ & 2.56 & $+/-0.37$ \\
\hline & Water & -2.11 & $+/-0.30$ & 2.91 & $+/-0.39$ \\
\hline & Tea & -1.99 & $+/-0.28$ & 2.41 & $+/-0.37$ \\
\hline
\end{tabular}

Estimated (adjusted) Means and associated 95\% confidence intervals per condition per comparison; ** Divided through by 60 for consistency with Affect Grid scale.

Table 3 IPANAT

\begin{tabular}{llcccc}
\hline \multirow{2}{*}{ Time } & \multirow{2}{*}{ Condition } & \multicolumn{2}{c}{ Positivity* $^{*}$} & \multicolumn{2}{c}{ Negativity } \\
\cline { 3 - 6 } & & M & SD & M & SD \\
\hline \multirow{3}{*}{$\mathbf{- 1 0}$} & Placebo & 2.18 & 0.44 & 1.99 & 0.49 \\
\cline { 2 - 6 } & Water & 2.12 & 0.43 & 2.12 & 0.37 \\
\cline { 2 - 6 }+5 & Tea & 2.00 & 0.51 & 1.89 & 0.40 \\
\cline { 2 - 6 } & Placebo & 2.08 & 0.45 & 1.98 & 0.51 \\
\hline & Water & 2.20 & 0.48 & 1.95 & 0.42 \\
\hline
\end{tabular}

* Significant at $p=0.05$ : higher after water versus placebo tea 
Fig. 1 Affect Grid: Mean (and SE) per dimension immediately after consumption (5min), adjusted for baseline (* Significant at $p=0.05$ )

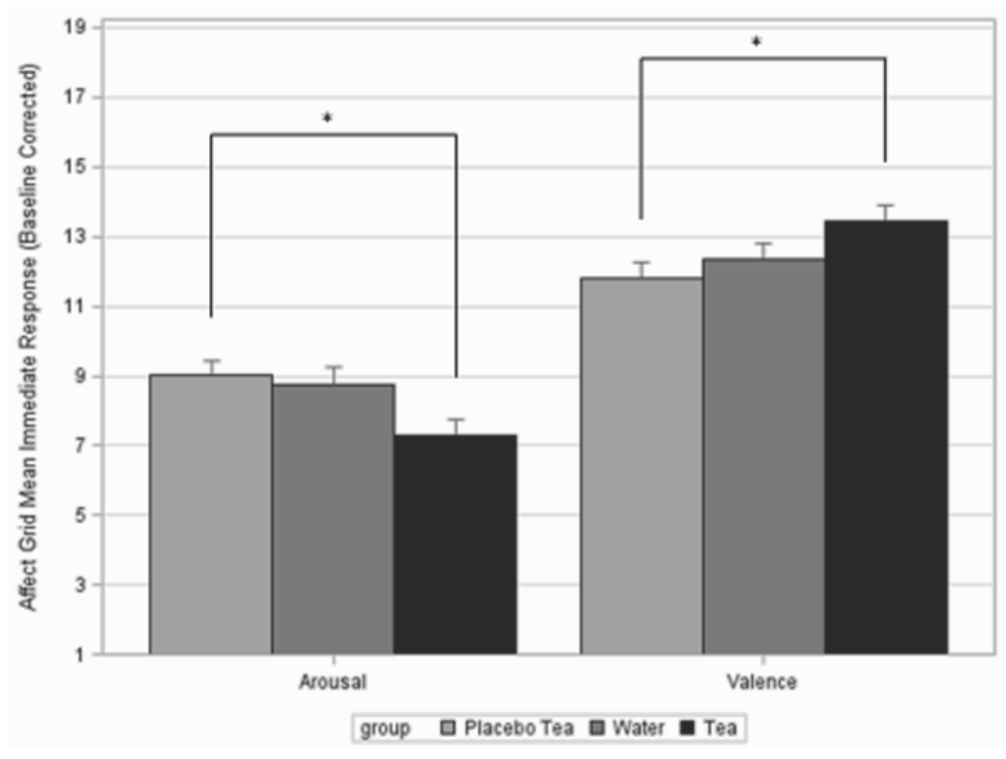

Fig. 2 MPAL: Mean score (and SE) per activity type

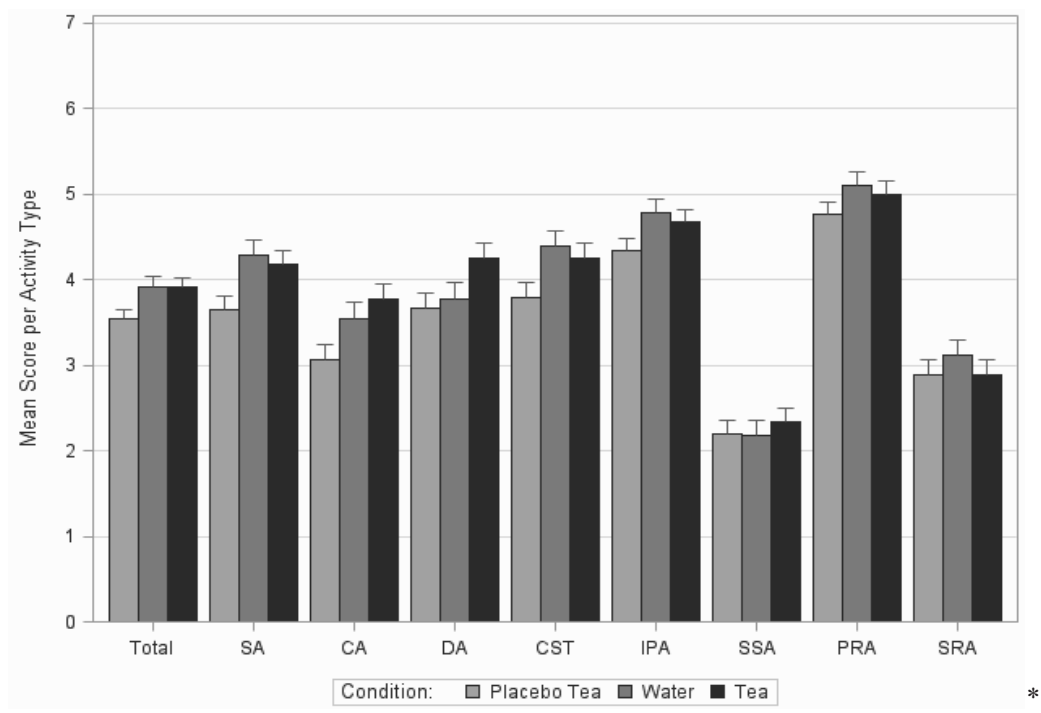



General Discussion

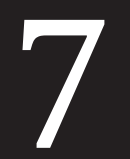


Across geographies and cultures, people share the belief that consumption of tea, particularly when made from the leaves of the Camellia Sinensis plant, provides mental benefits, such as feeling relaxed or energized and clarity of mind (Graham, 1992; Shimbo, Nakamura, Jing, Kizuki, Seino et al., 2005). The aim of this thesis was to further investigate whether consumption of tea, and/or specific tea ingredients, can be linked to acute mental benefits, using validated scientific measures.

In Chapter 1, the acute mental benefits of tea consumption were described in a framework consisting of two dimensions (i.e., cognitive and affective), and three postconsumption stages, notably immediately after consumption (i.e., 5 to 10 minutes), shortly after consumption (i.e., 10 to 30 minutes), and 30 minutes or more after consumption. We investigated the acute mental benefits of tea and/or its ingredients theanine and caffeine, in the light of this framework (see Fig. 1). Chapter 2 and 3 focused on cognitive effects of the consumption of theanine and caffeine, 30 minutes or more after consumption. Chapter 4 reviewed the research on both cognitive and affective benefits of tea and both tea ingredients to date. Chapter $\mathbf{5}$ and $\mathbf{6}$ focused on the affective benefits of a single cup of tea: Chapter 5 investigated immediate effects, while Chapter 6 included both immediate effects as well as effects over time.

\section{Fig. 1 Schematic overview of thesis}

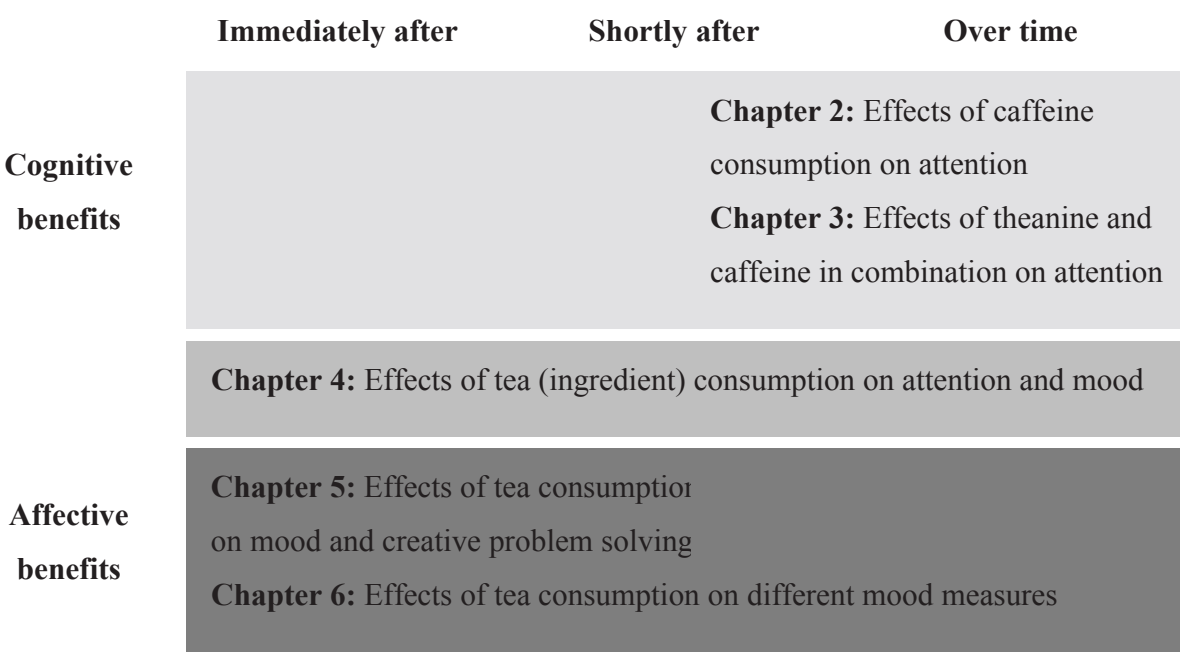




\section{SUMMARY AND DISCUSSION}

In Chapter 2, we discussed a comprehensive review of the literature with respect to the acute effects of caffeine consumption on attention performance, covering 66 individual studies. A number of specific hypotheses around the effects of caffeine were investigated. On the basis of the known mechanism of action for caffeine, the first hypothesis was that caffeine would demonstrate a general effect spanning different attention domains, rather than affecting specific attention domains. To investigate this hypothesis, we characterised each individual attention task in the studies reviewed according to two taxonomies of attention. The first taxonomy was the traditional division between simple and complex attention processes. The second was a taxonomy that distinguishes three functionally and anatomically different domains of attention: alerting (i.e., the ability achieve and maintain high sensitivity to incoming stimuli), orienting (i.e., the ability to selectively attend by directing attention to cued areas), and executive control (i.e., the ability to resolve conflict among thoughts, feelings, and responses). Findings confirmed that caffeine affected both simple and complex attention tasks. Effects of caffeine on complex attention did not appear to solely depend on faster processing, as effects extended beyond improved response speed to improved accuracy. Moreover, caffeine increased performance on tasks in both the alerting and executive function domains, but to a lesser extent on tasks in the orienting domain. However, it must be noted that few orienting tasks were used overall, which prevented drawing definitive conclusions on this domain.

We further hypothesised that higher caffeine doses would lead to stronger attention effects. While effects occurred even at low doses (i.e., $50 \mathrm{mg}$, which is similar to one to two cups of tea), there were indications of an optimal effect at doses around 200 mg. However, few studies directly compared multiple doses in a within subject design and the few that did yielded inconsistent results. Next, we investigated the hypothesis that habitual caffeine users would require a higher dose of caffeine as compared to nonusers of caffeine, in order to experience a comparable attention benefit. Evidence suggests that habitual caffeine consumption increases the number of adenosine receptors in the brain and hence more caffeine is needed to block them. Interestingly, we found that 
in some studies more pronounced effects of caffeine were demonstrated in high habitual users. Finally, we hypothesised that effects of acute caffeine consumption on attention are not influenced by withdrawal. We provided tentative evidence to support this hypothesis. It is worth noting that the hypotheses regarding caffeine doses and withdrawal could not be confirmed or disconfirmed conclusively, due to the considerable amount of variation within studies.

In sum, the current literature review provided clear answers to some of the key questions regarding caffeine as an attention enhancer. Its attention effects appear to be more widespread across different attention domains than previously assumed. However, other questions regarding dose-response effects and the role of habitual consumption and withdrawal, were more difficult to answer. In addition, the majority of research to date has focused on acute effects of single doses, which precludes conclusions on the build-up of effects when caffeine is consumed throughout the day, as is common practice for many of its consumers. The learnings from this review do provide a context in which to view the results of the studies on tea and theanine and caffeine in combination. Specifically, the review shows that consumption of a caffeine containing drink such as tea, would likely improve different facets of attention, when consumed in doses as commonly consumed during the day (i.e. 2 to 4 cups of tea), and that each cup consumed can benefit even the habitual caffeine consumer in a noticeable manner.

The study presented in Chapter 3 investigated the effects of an iced tea beverage containing a combination of theanine and caffeine, on attention. Assessments of attention performance and subjective attention (i.e., perceived alertness) were taken at baseline and starting 10 and 60 minutes after consumption of the theanine/ caffeine beverage and a placebo. The beverage contained an enhanced dose of theanine relative to caffeine; 50 $\mathrm{mg}$ caffeine (i.e., similar to the amount in two cups of tea) and $100 \mathrm{mg}$ theanine (i.e., similar to the amount in five cups of tea). It was compared to a placebo drink without theanine and caffeine. The aim was to gain further insights on the benefits of the theanine/ caffeine combination, by seeking to replicate and extend the findings of an earlier study (Owen et al., 2008). We confirmed that the combination increased the number of correct responses on a switch task (Rogers \& Monsell, 1995), which required switching between task sets. Also, tentative evidence was found that the combination 
improved performance on an intersensory task (Gomez-Ramirez et al., 2007), which required orienting attention to visual or auditory stimuli. The combination did not affect the subjective manifestation of attention, i.e. feelings of alertness.

Findings supported earlier evidence on the effects of theanine in combination with caffeine on switch task performance (Giesbrecht et al., 2010; Haskell et al., 2008; Owen et al., 2008). Moreover, findings could be extended to performance on a different complex attention task; the intersensory task. However, effects on this task were smaller. Therefore, one might argue that the theanine/ caffeine combination improves executive control and alerting to a greater extent than orienting of attention. This would be in line with findings from our caffeine review (Chapter 2). Unlike in the aforementioned previous studies, the combination did not enhance feelings of alertness. Whereas, caffeine predominantly yields arousing effects, a high dose of theanine may counteract these effects, therefore allowing for improved performance on higher-order tasks but not higher perceived alertness. The main limitation of the present study is that we cannot conclude on synergistic effects between theanine and caffeine, or the effect of theanine on its own, as ingredients were not tested in isolation. Also, we cannot directly conclude on tea consumption, as theanine levels were enhanced by five-fold. However, combined with the evidence to date, the research in Chapter 3 does suggest that a beverage containing the combination of theanine and caffeine, can improve certain aspects of attention.

In Chapter 4, we reviewed the research to date on the acute effects of tea consumption, both cognitive and affective. Moreover, we included specific reference to its ingredients theanine and caffeine, as well as outcomes reflecting real-life contexts where attention is important (i.e., at work, while driving and in military settings). To date, four studies assessed effects of tea consumption on cognition, nine assessed mood effects of tea, and six assessed effects of a combination of caffeine and theanine, mainly on cognition and perceived alertness. With regard to cognitive benefits, it was concluded that tea, and tea ingredients theanine and caffeine in combination consistently improve attention and self-reported alertness acutely starting around 30 minutes post-consumption. Also, a handful of studies demonstrates promising findings that the benefits of tea may translate to real life scenarios. 
Affective benefits of tea, beyond alertness, have been less extensively researched and findings are mixed. A similar pattern of mixed findings was also found for studies on theanine and caffeine in combination. Moreover, studies focused on effects that are experienced 30 minutes after consumption of tea or later, in line with the research on cognitive effects. Hence, there is no research regarding immediate affective effects. Some studies reported increased feelings of pleasure after tea consumption compared to water (Quinlan et al., 1997), while others did not (De Bruin et al., 2011; Quinlan et al., 2000). Interestingly, while tea is typically associated with relaxation and theanine has been shown to affect activity in the alpha wave of the EEG spectrum (Nobre, Rao, \& Owen, 2008), which is related to being in state of relaxation, no studies have directly assessed the acute effects of tea on self-reported relaxation.

We also concluded that studies to date largely neglected the possibility that mood effects of tea may occur during and/or immediately after consumption, due to the consumption experience itself. This consumption experience includes the combination of the tea preparation, sensory properties and potentially even simply providing a break from other activities. Moreover, short-term improvements in mood have been related to longer-term well-being, motivational and cognitive benefits. This is of interest, as it may help us to understand what brief uplifts in mood could potentially deliver in our day-to-day lives.

Chapter 5 pertains to the first investigation of the affective benefits of a cup of tea immediately after consumption. In a parallel design, we compared the preparation and consumption of tea, to consumption of water and a validated positive affect induction (i.e., happy memory recall). The primary aim was to assess immediate effects on arousal and valence of mood. An area which has recently received a lot of attention, is the relation between positive affect and improved creativity, and we were interested to see whether improved mood from tea consumption could improve creativity. Hence, our secondary aim was to assess effects on a creative problem solving task (convergent creativity) and a creative drawing task (divergent creativity). We found that tea consumption enhanced the valence dimension of mood, indicating greater feelings of pleasure. This effect was significant as compared to water, and was similar to the effect of 
the affect induction. Tea (and water) consumption reduced the arousal dimension of mood, as compared to the affect induction, indicating slightly increased relaxation.

We also found tentative evidence that tea consumption and the affect induction lead to faster responses for difficult problems in the creative problem solving task. This could indicate that positive affect from tea (and the positive control) may lead to faster insights, indicative of more associative and flexible processing (De Dreu, Baas, \& Nijstad, 2008). There were no difference between conditions in originality of drawings. This may indicate a specificity of effects on convergent creativity rather than divergent creativity, but also that effects may be short-lived, as the creative problem solving task was always completed first.

In sum, we concluded that a simple everyday activity such as tea consumption can effectively boost pleasure immediately after consumption. Also, this study takes a first step to understanding how mood benefits could potentially result in subsequent behaviour and improved performance, for example in the form of creativity. However, it remains to be disentangled which aspect(s) of tea consumption led to the reported changes in valence of mood, as sensory aspects such as taste and aroma, consumption expectations, and even the tea preparation itself may be contributing to this effect.

Finally, Chapter 6 further investigated the affective benefits of consuming a cup of tea, immediately after consumption as well as over time. Consumption of tea was compared to consumption of cold water and a placebo tea, in a parallel design. This design allowed a comparison to the previous study (Chapter 5) regarding immediate valence effects of tea as compared to water, as well a comparison to studies on tea and the theanine/ caffeine combination (Chapter 3 to 4), regarding arousal effects later in time as compared to placebo. Mood effects were explored using two different frameworks, i.e. the bipolar framework of valence and arousal (Russell, 1989), and the dimensional framework of discrete emotions (Ekman, 1992). In addition, two measures of implicit mood were included. Immediately after consumption, tea increased valence and reduced arousal as compared to placebo tea, but not compared to water. There were no differences related to tea in discrete emotions evoked, or in implicit positivity or negativity. Consumption of tea and water did increase interest in activities overall and specific types of activities, as compared to placebo. Specifically, tea consumption resulted in 
higher interest in creative and domestic activities, while water resulted in higher interest in social activities, and culture, science and travel related activities.

We concluded that mood effects of a single cup of tea appeared shortly after consumption but were not sustained over time. In contrast to Chapter 5, differences between tea and water, albeit in the same direction, were not significant. This could be explained by the lack of preparation and the standardized unbranded tea in the current study. Consumption pleasure may depend on personal preferences in strength and brewing time, and anticipation as well as simple preparation rituals can further enhance pleasure experienced. In addition, the products in this study were unbranded in order to allow a blind comparison between the true tea and the placebo tea. We know however that a congruent consumption context, including a familiar brand can enhance positive affect (Isen et al., 2004). Moreover, consumption of a single cup may not be sufficient to stimulate arousal and may actually lead to reduced arousal immediately after consumption.

We further concluded that discrete emotions may be of greater relevance when addressing the social and situational consumption context in everyday life, rather than the current semi-lab based study. The increased interest in activity after tea (and to some extent water) consumption, further strengthens the idea that mood benefits from tea consumption could have relevance for subsequent behaviour and performance. Finally, it would be advisable for future studies to focus on the immediate consumption moment, including preparation and a congruent and relevant context or need state. 


\section{IMPLICATIONS}

Based on the findings presented in this thesis, we can conclude that consumption of (black) tea has acute cognitive and affective benefits. However, some of these effects follow a different temporal profile due to their underlying mechanism of action.

Cognitive benefits of tea consumption appear to be largely driven by its ingredients theanine and caffeine. The nature of these effects is clear and consistent: from around 30 minutes after the consumption of tea, or a combination of caffeine and theanine, attention performance was improved on a range of attention tasks (i.e., simple and complex attention, and alerting and executive function). In addition to these improvements in objective measures of attention, subjective alertness was also improved, as indicated by a selection of questionnaires. These findings are in line with consumer beliefs regarding mental clarity and alertness from tea consumption.

Evidence for affective benefits, beyond alertness is limited. Some effects, like feelings of pleasure, are experienced immediately after consumption and are of a rather short duration. These seem to be due to the consumption experience itself, rather than due to pharmacological effects of tea ingredients. Mood effects over time, potentially induced by tea ingredients, were found to be inconsistent in the research to date. However, this may have been due to the variation in study designs. Moreover, the prevalence and size of the immediate mood effects of tea seems to rely heavily on factors such as the product preparation, a congruent consumption context and the amount of servings consumed. We have made a first attempt to capture some of these factors, but they remain to be explored. Hence, there seems to be some tentative evidence for consumer beliefs around relaxation and positive emotions following tea consumption, but this warrants further investigations, preferably in relevant and congruent settings.

\section{Cognitive benefits}

Attention effects of tea and its ingredients appeared consistently. However, there are few remaining questions, regarding scope and size of these effects. Findings on the scope of the cognitive effects seem promising in that the attention effect of tea and tea ingredients is not restricted to specific attention tasks, but seems rather generic. Fur- 
thermore, the small number of studies conducted outside the laboratory have shown promising results, but were limited to caffeine (Brice \& Smith, 2001; Ker et al., 2010; McLellan et al., 2005a; McLellan et al., 2005b; Peeling \& Dawson, 2007). An exception is a cross-sectional study that showed benefits of tea consumption on self-reported work performance during 10 workdays (Bryan et al., 2012). Laboratory tasks possess the rigor and control needed to demonstrate causal effects of interventions, and most scientists would argue that findings from such laboratory studies can serve to explain and predict behaviour. However, real-life tasks and field studies can help to complement laboratory findings, and can give a more complete picture of how cognitive processes translate into complex everyday behaviours. Therefore, it is highly relevant to better understand the effects of the consumption of tea (or tea ingredients) on real life activities requiring attention.

Also, tea consumption is not isolated but part of a greater set of consumption and behaviour routines. Thus, it would be highly relevant to understand the effect of single, or multiple consumptions of tea within naturalistic consumption patterns. Future studies could benefit from researching daylong consumption of tea and other beverages in a naturalistic setting (e.g. at home and work), which includes commons factors such as stress that can affect mental performance, mood and/or wellbeing. Moreover, different individuals may vary in sensitivity to and/or their need for the effects of tea ingredients, in particular caffeine, and dose-response relationships between dose and cognitive benefits is not yet known. This will allow us to further understand the build-up and patterns of effects when consumed throughout the day, as is commonly seen.

With regard to the size of the effect, the findings in this thesis provided limited insights. A recent publication reviewed 11 studies, including studies on the theanine/ caffeine combination reported in Chapter 3, and the tea studies in Chapter 4 (Camfield et al., 2014). The authors conducted a set of meta-analyses on the switch task (5 studies) and the intersensory task (4 studies), with specific questions regarding the effects during the first and the second hour post-consumption (analysis of mood data is discussed in the next section). They reported small to medium effect sizes for switch accuracy, and small effect sizes for intersensory accuracy, in favour of tea. However, the authors failed to observe significant effects for caffeine or theanine dose. They concluded that theanine and caffeine in combination enhance attention and warn that some effects may 
have been underestimated due to the time frame studied. To optimally capture effects, future studies could be better tailored to the time needed for these ingredients to reach peak plasma levels (i.e. caffeine: 30 minutes; theanine: 50 minutes), or ideally peak brain levels.

A final note considering the small to medium size of effects, is that one should keep in mind that the product under investigation is a natural food product, which can be consumed safely on multiple occasions daily as part of a normal diet. As such, cognitive effects will not be of the same magnitude as drugs or medications. However, scope and size of the current effects does seem to fit with the health and wellness trends around food and beverages consumption; i.e. with the growing daily demands, people are looking for ways to stay mentally alert, in a natural way.

\section{Affective benefits}

Our studies have shown some immediate and rather general affective benefits of tea on immediate feelings of pleasure, but effects over time on remain inconsistent in the research to date. The meta-analysis by Camfield et al. (2014) confirms these findings, as there were no significant effects for calmness, contentedness or anxiety after tea consumption . However, there was a suggestion of a trend where calmness ratings decreased as caffeine dose increased, and calmness ratings increased as the theanine dose increased. Questions remain with regard to the role of context, the influence of daily patterns, and disentangling the crucial parts of the tea experience itself.

People indeed report to eat, drink, or smoke to feel good and different groups of people may select different beverages in general, and for specific occasions or in specific contexts. Perhaps this is based on their experience with and/or beliefs around the effects of these beverages and the need state that they are in when they choose a particular beverage. However, the context of consumption was not included in any of the studies to date. Not surprisingly, the frequency and intensity of positive emotions to food are generally higher in appropriate contexts (Piqueras-Fiszman \& Jaeger, 2015). Therefore, to better understand the affective benefits of the tea consumption experience, future studies should employ a more holistic approach, including a situational, emotional, physical and/or social context. 
Moreover, although some psychological (and related physiological) patterns seem to occur in relation to black tea consumption, to date these have not been observed in a continuous manner (e.g., throughout the day) in real-life settings, taking into account common mood fluctuations during the day and between days. Only one study investigated the relation between beverage consumption and mood over time, and with promising results (Bryan et al., 2012). Participants who consumed more tea reported feeling less tired and performing better. Future research could benefit from employing an observational setting, for example at work, and using non-intrusive continuous monitoring of psychological as well as physiological outcomes.

Finally, the role of different aspects of the tea experience could be further investigated. In addition to factors mentioned earlier (i.e., preparation, a congruent and relevant consumption context), we can identify a few other relevant ones, such as expectations and sensorial aspects. When interacting with any food product or beverage, we integrate the information perceived from the product with the information that we have stored from previous experiences of the same or a similar product, to form our expectations of that product. Expectations are considered crucial in the liking and acceptance of food products, and may also be partially responsible for some of the effects demonstrated, both cognitive and affective. For example, studies have demonstrated effects of the expectation of having consumed caffeine (Dawkins, Shahzad, Ahmed, \& Edmonds, 2011), and taste expectations raised by packaging and sensory properties (Piqueras-Fiszman \& Spence, 2015).

In addition, the role of the different sensory aspects in the consumption experience remains unknown. Tea has a number of distinctive sensory properties such as the temperature at which it is drunk, its taste, astringency, and aroma. Not only is black tea highly aromatic during consumption, pouring hot water on (a bag containing) tea leaves also leads to an immediate aroma release prior to consumption. Interestingly, aromas can have a direct effect on emotions, likely due to the overlap in the brain areas associated with olfaction and emotion (Royet, Plailly, Delon-Martin, Kareken, Segebarth, et al. 2003; Rolls 2004). Moreover, recent studies suggest a role for specific aroma volatiles from green tea or so-called "green odours" such as those from the tea plant, in stress reduction and relaxation (Murao, Yoto, \& Yokogoshi, 2013). However, there is no research to date into the role of tea aroma (or its other sensory properties) in relation to the tea experience. 


\section{CONCLUSION}

Tea has historically been associated with mental benefits. The aim of this thesis was to further investigate whether consumption of tea, and/or specific tea ingredients theanine and caffeine, provides acute cognitive and affective benefits. In a review of the literature, we found that attention effects of caffeine were more pronounced than previously assumed. In our first study, we showed that attention performance was improved by consuming a drink containing a combination of theanine and caffeine, as compared to a placebo drink. A second review concluded that consumption of tea, and theanine and caffeine in combination, consistently improves attention and self-reported alertness. Other and more immediate affective benefits of tea consumption were not extensively researched and were less consistent. A second study found that tea consumption (and preparation) improved valence of mood immediately after consumption, as compared to water consumption, and may improve creative problem solving. The final study confirmed that mood effects of a single cup of tea appeared shortly after consumption, but were not sustained over time. Also, tea resulted in increased interest in activities overall and specific types of activities, as compared to placebo. Based on the combined evidence presented in this thesis, we conclude that converging evidence from (neuro-) physiological, behavioural, and self-report-based outcomes lends credibility to the idea that tea consumption is associated with cognitive and affective benefits, as widely believed. 

References 
Adan, A., \& Serra-Grabulosa, J. M. (2010). Effects of caffeine and glucose, alone and combined, on cognitive performance. Human Psychopharmacology, 25, 310-317.

Addicott, M. A., \& Laurienti, P. J. (2009). A comparison of the effects of caffeine following abstinence and normal caffeine use. Psychopharmacology, 207, 423-31.

Amendola, C. A., Gabrieli, J. D. E., \& Lieberman, H. R. (1998). Caffeine's effects on performance and mood are independent of age and gender. Nutritional Neuroscience, 1, 269-280.

Appleton, M., \& Rogers, P. J. (2004). Food and Mood. Womens Health Medicine, 1, 4-6.

Arab, L., Biggs, M. L., O'Meara, E. S., Longstreth, W. T., Crane, P. K., \& Fitzpatrick, A. L. (2011). Gender differences in tea, coffee, and cognitive decline in the elderly: the Cardiovascular Health Study. Journal of Alzheimer's Disease, 27, 553-566.

Ashby, F. G., Isen, A. M., \& Turken, A. U. (1999). A neuropsychological theory of positive affect and its influence on cognition. Psychological Review, 106, 529-550.

Aspen, J., \& Quinlan, P. T. (1998). The immediate alerting effects of hot beverage ingestion: mediated by caffeine or sensory factors? Chemical Senses, 23, 561-562.

Attwood, A. S., Higgs, S., \& Terry, P. (2007). Differential responsiveness to caffeine and perceived effects of caffeine in moderate and high regular caffeine consumers. Psychopharmacology, 190, 469-477.

Baas, M., De Dreu, C. K., \& Nijstad, B. A. (2008). A meta-analysis of 25 years of mood-creativity research: hedonic tone, activation, or regulatory focus? Psychological Bulletin, 134, 779-806.

Baas, M., De Dreu, C. K., \& Nijstad, B. A. (2011). When prevention promotes creativity: the role of mood, regulatory focus, and regulatory closure. Journal of Personality and Social Psychology, 100, 794809.

Barry, R. J., Johnstone, S. J., Clarke, A. R., Rushby, J. A., Brown, C. R., \& McKenzie, D. N. (2007). Caffeine effects on ERPs and performance in an auditory Go/NoGo task. Clinical Neurophysiology, 118, 2692-2699.

Beaven, C. M., Maulder, P., Pooley, A., Kilduff, L., \& Cook, C. (2013). Effects of caffeine and carbohydrate mouth rinses on repeated sprint performance. Applied Physiology, Nutrition, and Metabolism, 38, 633-637.

Bennet, G. (1988). Eating Matters: Why we eat what we eat. London: Heinemann.

Benton, D., \& Young, H. A. (2015). Do small differences in hydration status affect mood and mental performance?. Nutrition reviews, 73, 83-96.

Berridge, K. C., \& Robinson, T. E. (2003). Parsing reward. Trends in Neuroscience, 26, 507-513.

Blanchard, J., \& Sawers, S. (1983). Comparative pharmacokinetics of caffeine in young and elderly men. Journal of Pharmacokinetics and Biopharmacology, 11, 26.

Bond, A., \& Lader, M. (1974). The use of analogue scales in rating subjective feelings. British Journal of Psychology, 47, 211-218.

Botvinick, M. M., Braver, T. S., Barch, D. M., Carter, C. S., \& Cohen, J. D. (2001). Conflict monitoring and cognitive control. Psychological Review, 108, 624-652.

Brice, C., \& Smith, A. (2001). The effects of caffeine on simulated driving, subjective alertness and sustained attention. Human Psychopharmacology, 16, 523-531.

Brown, F., \& Diller, K. R. (2008). Calculating the optimum temperature for serving hot beverages. Burns, 34, 648-654.

Bruce, M., Scott, N., Lader, M., \& Marks, V. (1986). The psychopharmacological and electrophysiological effects of single doses of caffeine in healthy human subjects. British Journal of Clinical Pharmacology, 22, 81-87.

Brunye, T. T., Mahoney, C. R., Lieberman, H. R., \& Taylor, H. A. (2010a). Caffeine modulates attention network function. Brain \& Cognition, 72, 181-188.

Brunye, T. T., Mahoney, C. R., Lieberman, H. R., Giles, G. E., \& Taylor, H. A. (2010b). Acute caffeine consumption enhances the executive control of visual attention in habitual consumers. Brain \& Cognition, 74, 186-192. 
Bryan, J. (2008). Psychological effects of dietary components of tea: caffeine and L-theanine. Nutrition reviews, 66, 82-90.

Bryan, J., Tuckey, M., Einother, S. J., Garczarek, U., Garrick, A., \& De Bruin, E. A. (2012). Relationships between tea and other beverage consumption to work performance and mood. Appetite, 58, 339-346.

Bush, G., Luu, P., \& Posner, M. I. (2000). Cognitive and emotional influences in anterior cingulate cortex. Trends in Cognitive Science, 4, 215-222.

Caldwell, J. A., \& Caldwell, J. L. (2005). Fatigue in military aviation: an overview of US military-approved pharmacological countermeasures. Aviation, Space and Environmental Medicine, 76, C39-C51.

Camfield, D. A., Stough, C., Farrimond, J., \& Scholey, A. B. (2014). Acute effects of tea constituents Ltheanine, caffeine, and epigallocatechin gallate on cognitive function and mood: a systematic review and meta-analysis. Nutrition reviews, 72, 507-522.

Canli, T., Omura, K., Haas, B. W., Fallgatter, A., Constable, R. T., \& Lesch, K. P. (2005). Beyond affect: a role for genetic variation of the serotonin transporter in neural activation during a cognitive attention task. Proceedings of the National Academy of Sciences, 102, 12229.

Canli, T., Qui, M., Omura, K., Condon, E., Haas, B. W., Amin, Z. et al. (2006). Neural correlates of epigenesis. Proceedings of the National Academy of Sciences, 103, 16038.

Chambers, E. S., Bridge, M. W., \& Jones, D. A. (2009). Carbohydrate sensing in the human mouth: effects on exercise performance and brain activity. Journal of Physiology, 587, 1779-1794.

Chen, M. (2003). Tea and Health - an overview. In Y.S. Zhen, Z. Chen, S. Cheng, \& M. Chen (Eds.), Tea: bioactivity and therapeutic potential (pp. 1-16). London: Taylor and Francis.

Childs, E., \& de, W. H. (2006). Subjective, behavioural, and physiological effects of acute caffeine in light, nondependent caffeine users. Psychopharmacology, 185, 514-523.

Childs, E., \& de, W. H. (2008). Enhanced mood and psychomotor performance by a caffeine-containing energy capsule in fatigued individuals. Experimental and Clinical Psychopharmacology, 16, 13-21.

Christie, I. C., \& Friedman, B. H. (2004). Autonomic specificity of discrete emotion and dimensions of affective space: a multivariate approach. International Journal of Psychophysiology, 51, 143-153.

Christopher, G., Sutherland, D., \& Smith, A. (2005). Effects of caffeine in non-withdrawn volunteers. Human Psychopharmacology, 20, 47-53.

Clore, G. L., \& Huntsinger, J. R. (2007). How emotions inform judgment and regulate thought. Trends in Cognitive Sciences, 11, 393-399.

Corbetta, M., Kincade, J. M., Ollinger, J. M., McAvoy, M. P., \& Shulman, G. L. (2000). Voluntary orienting is dissociated from target detection in human posterior parietal cortex. Nature Neuroscience, 3, 292297.

Cunningham, M. R. (1988a). Does happiness mean friendliness? Induced mood and heterosexual selfdisclosure. Personality and Social Psychology Bulletin, 14, 283-297.

Cunningham, M. R. (1988b). What do you do when you're happy or blue? Mood, expectancies, and behavioural interest. Motivation and Emotion, 12, 331.

Dager, S. R., \& Friedman, S. D. (2000). Brain imaging and the effects of caffeine and nicotine. Annals of Medicine, 32, 599.

Daly, J. W., Jacobson, K. A., \& Ukena, D. (1987). Adenosine receptors: development of selective agonists and antagonists. Progress in Clinical and Biological Research, 230, 41-63.

Daval, J. L., Von Lubitz, D. K., Deckert, J., \& Marangos, P. J. (1989). Protective effects of cyclohexyladenosine following cerebral ischemia in the gerbil hippocampus. Advances in Experimental Medicine and Biology, 253B, 447-454.

Davis, M. A. (2009). Understanding the relationship between mood and creativity: A meta-analysis. Organizational Behaviour and Human Decision Processes, 108, 25-38.

Dawkins, L., Shahzad, F. Z., Ahmed, S. S., \& Edmonds, C. J. (2011). Expectation of having consumed caffeine can improve performance and mood. Appetite, 57, 597-600.

De Bruin, E. A., \& Gilsenan, M. B. (2009). Effects of food energy on cognitive performance: no support from event-related potentials (yet?). British Journal of Nutrition, 101, 1047-1051. 
De Bruin, E. A., Rowson, M. J., Van Buren, L., Rycroft, J. A., \& Owen, G. N. (2011). Black tea improves attention and self-reported alertness. Appetite, 56, 235-240.

De Dreu, C. K., Baas, M., \& Nijstad, B. A. (2008). Hedonic tone and activation level in the mood-creativity link: toward a dual pathway to creativity model. Journal of Personality and Social Psychology, 94, 739-756.

Deslandes, A. C., Veiga, H., Cagy, M., Piedade, R., Pompeu, F., \& Ribeiro, P. (2005). Effects of caffeine on the electrophysiological, cognitive and motor responses of the central nervous system. Brazilian Journal of Medical and Biological Research, 38, 1077-1086.

Desmet, P. M. A., \& Schifferstein, H. N. J. (2008). Sources of positive and negative emotions in food experience. Appetite, 50, 290-301.

Dijkstra, J. J. (1999). User agreement with incorrect expert system advice. Behaviour \& Information Technology, 18, 399-411.

Dohle, S., Rall, S., \& Siegrist, M. (2014). I cooked it myself: Preparing food increases liking and consumption. Food Quality and Preference, 33, 16.

Dufresne, C. J., \& Farnworth, E. R. (2001). A review of latest research findings on the health promotion properties of tea. Journal of Nutritional Biochemistry, 12, 404-421.

Durlach, P. J. (1998). The effects of a low dose of caffeine on cognitive performance. Psychopharmacology, 140, 116-119.

Edmonds, C. J., Crombie, R., Ballieux, H., Gardner, M. R., \& Dawkins, L. (2013). Water consumption, not expectancies about water consumption, affects cognitive performance in adults. Appetite, 60, 148-153.

Einöther, S. J. L., \& Giesbrecht, T. (2013). Caffeine as an attention enhancer: reviewing existing assumptions. Psychopharmacology, 225, 274.

Einöther, S. J. L., \& Martens, V. E. G. (2013). Acute effects of tea consumption on attention and mood. American Journal of Clinical Nutrition, 98, 1700S-1708S.

Einöther, S. J. L., Baas, M., Rowson, M., \& Giesbrecht, T. (2015). Investigating the effects of tea, water and a positive affect induction on mood and creativity. Food Quality and Preference, 39, 56-61.

Einöther, S. J. L., Martens, V. E., Rycroft, J. A., \& De Bruin, E. A. (2010). L-theanine and caffeine improve task switching but not intersensory attention or subjective alertness. Appetite, 54, 406-409.

Ekman, P. (1992). An argument for basic emotions. Cognition and Emotion, 6, 169-200.

Estrada, C. A., Young, M., \& Isen, A. M. (1994). Positive affect influences creative problems solving and reported source of practice satisfaction in physicians. Motivation and Emotion, 18, 285-299.

Fagan, D., Swift, C. G., \& Tiplady, B. (1988). Effects of caffeine on vigilance and other performance tests in normal subjects. Journal of Psychopharmacology, 2, 19-25.

Fan, J., Fossella, J., Sommer, T., Wu, Y., \& Posner, M. I. (2003). Mapping the genetic variation of executive attention onto brain activity. Proceedings of the National Academy of Sciences, 100, 7406-7411.

Fan, J., McCandliss, B. D., Fossella, J., Flombaum, J. I., \& Posner, M. I. (2005). The activation of attentional networks. Neuroimage, 26, 471-479.

Fan, J., McCandliss, B. D., Sommer, T., Raz, A., \& Posner, M. I. (2002). Testing the efficiency and independence of attentional networks. Journal of Cognitive Neuroscience, 14, 340-347.

Fastbom, J., Post, C., \& Fredholm, B. B. (1990). Antinociceptive effects and spinal distribution of two adenosine receptor agonists after intrathecal administration. Pharmacology and Toxicology, 66, 69-72.

Feng, L., Gwee, X., Kua, E. H., \& Ng, T. P. (2010). Cognitive function and tea consumption in community dwelling older Chinese in Singapore. The Journal of Nutrition Health and Aging, 14, 433-438.

Feng, L., Li, J., Kua, E. H., Lee, T. S., Yap, K. B., John, R. A. et al. (2012). Association between tea consumption and depressive symptoms in older Chinese adults. Journal of the American Geriatric Society, 60, 2358-2360.

Ferre, S. (2008). An update on the mechanisms of the psychostimulant effects of caffeine. Journal of Neurochemistry, 105, 1067-1079.

Ferre, S. (2010). Role of the central ascending neurotransmitter systems in the psychostimulant effects of caffeine. Journal of Alzheimer's Disease, 20, S35-S49. 
Ferre, S., Fredholm, B. B., Morelli, M., Popoli, P., \& Fuxe, K. (1997). Adenosine-dopamine receptor-receptor interactions as an integrative mechanism in the basal ganglia. Trends in Neuroscience, 20, 482-487.

Ferre, S., Fuxe, K., von, E. G., Johansson, B., \& Fredholm, B. B. (1992). Adenosine-dopamine interactions in the brain. Neuroscience, 51, 501-512.

Fine, B. J., Kobrick, J. L., Lieberman, H. R., Marlowe, B., Riley, R. H., \& Tharion, W. J. (1994). Effects of caffeine or diphenhydramine on visual vigilance. Psychopharmacology, 114, 233-238.

Fisone, G., Borgkvist, A., \& Usiello, A. (2004). Caffeine as a psychomotor stimulant: mechanism of action. Cellular and Molecular Life Sciences, 61, 857-872.

Foskett, A., Ali, A., \& Gant, N. (2009). Caffeine enhances cognitive function and skill performance during simulated soccer activity. International Journal of Sport Nutrition and Exercise Metabolism, 19, 410-423.

Foxe, J. J., Morie, K. P., Laud, P. J., Rowson, M. J., De Bruin, E. A., \& Kelly, S. P. (2012). Assessing the effects of caffeine and theanine on the maintenance of vigilance during a sustained attention task. Neuropharmacology, 62, 2320-2327.

Foxe, J. J., Simpson, G. V., \& Ahlfors, S. P. (1998). Parieto-occipital $\sim 10 \mathrm{~Hz}$ activity reflects anticipatory state of visual attention mechanisms. Neuroreport, 9, 3929-3933.

Fredrickson, B. L. (2001). The role of positive emotions in positive psychology. The broaden-and-build theory of positive emotions. American Psychologist, 56, 218-226.

Fredrickson, B. L. (2004). The broaden-and-build theory of positive emotions. Philosophical Transactions of the Royal Society B: Biological Sciences, 359, 1367-1378.

Friedman, R., \& Elliott, A. J. (2008). The effect of arm crossing on persistence and performance. European Journal of Social Psychology, 38, 449-461.

Giesbrecht, T., Rycroft, J. A., Rowson, M. J., \& De Bruin, E. A. (2010). The combination of L-theanine and caffeine improves cognitive performance and increases subjective alertness. Nutritional Neuroscience, 13, 283-290.

Giles, G. E., Mahoney, C. R., Brunye, T. T., Gardony, A. L., Taylor, H. A., \& Kanarek, R. B. (2012). Differential cognitive effects of energy drink ingredients: caffeine, taurine, and glucose. Pharmacology, Biochemistry and Behaviour, 102, 569-577.

Gomez-Ramirez, M., Higgins, B. A., Rycroft, J. A., Owen, G. N., Mahoney, J., Shpaner, M. et al. (2007). The deployment of intersensory selective attention: a high-density electrical mapping study of the effects of theanine. Clinical Neuropharmacology, 30, 25-38.

Gomez-Ramirez, M., Kelly, S. P., Montesi, J. L., \& Foxe, J. J. (2009). The Effects of L-theanine on alphaband oscillatory brain activity during a visuo-spatial attention task. Brain Topography, 22, 44-51.

Graham, H. N. (1992). Green tea composition, consumption, and polyphenol chemistry. Preventive Medicine, 21, 334-350.

Gutjar, S., de Graaf, C., Kooijman, V., de Wijk, R. A., Nys, A., ter Horst, G. J. et al. (2015). The role of emotions in food choice and liking. Food Research International, 76, 216-223.

Hasenfratz, M., \& Battig, K. (1992). Action profiles of smoking and caffeine: Stroop effect, EEG, and peripheral physiology. Pharmacology, Biochemistry and Behaviour, 42, 155-161.

Hasenfratz, M., \& Battig, K. (1994). Acute dose-effect relationships of caffeine and mental performance, EEG, cardiovascular and subjective parameters. Psychopharmacology, 114, 281-287.

Haskell, C. F., Kennedy, D. O., Milne, A. L., Wesnes, K. A., \& Scholey, A. B. (2008). The effects of 1theanine, caffeine and their combination on cognition and mood. Biological Psychology, 77, 113-122.

Haskell, C. F., Kennedy, D. O., Wesnes, K. A., \& Scholey, A. B. (2005). Cognitive and mood improvements of caffeine in habitual consumers and habitual non-consumers of caffeine. Psychopharmacology, $179,813-825$.

Heatherley, S. V., Hayward, R. C., Hill, J., Smit, H. J., Cater, K. F., \& Rogers, P. J. (2004). Effects of caffeine and caffeine withdrawal on simulated driving performance. Journal of Psychopharmacology, 18, A29.

Heatherley, S. V., Hayward, R. C., Seers, H. E., \& Rogers, P. J. (2005). Cognitive and psychomotor performance, mood, and pressor effects of caffeine after 4, 6 and $8 \mathrm{~h}$ caffeine abstinence. Psychopharmacology, 178, 461-470. 
Heckman, M. A., Weil, J., \& Gonzalez de, M. E. (2010). Caffeine (1, 3, 7-trimethylxanthine) in foods: a comprehensive review on consumption, functionality, safety, and regulatory matters. Journal of Food Science, 75, R77-R87.

Heiss, M. L., \& Heiss, R. J. (2007). The Story of Tea: A Cultural History and Drinking Guide. Berkeley, California: Ten Speed Press.

Hewlett, P., \& Smith, A. (2006). Acute effects of caffeine in volunteers with different patterns of regular consumption. Human Psychopharmacology, 21, 167-180.

Hewlett, P., \& Smith, A. (2007). Effects of repeated doses of caffeine on performance and alertness: new data and secondary analyses. Human Psychopharmacology, 22, 339-350.

Hindmarch, I., Quinlan, P. T., Moore, K. L., \& Parkin, C. (1998). The effects of black tea and other beverages on aspects of cognition and psychomotor performance. Psychopharmacology, 139, 230-238.

Hindmarch, I., Rigney, U., Stanley, N., Quinlan, P., Rycroft, J., \& Lane, J. (2000). A naturalistic investigation of the effects of day-long consumption of tea, coffee and water on alertness, sleep onset and sleep quality. Psychopharmacology, 149, 203-216.

Horne, J. A., Reyner, L. A., \& Barrett, P. R. (2003). Driving impairment due to sleepiness is exacerbated by low alcohol intake. Occupational and Environmental Medicine, 60, 689-692.

Hozawa, A., Kuriyama, S., Nakaya, N., Ohmori-Matsuda, K., Kakizaki, M., Sone, T. et al. (2009). Green tea consumption is associated with lower psychological distress in a general population: the Ohsaki Cohort 2006 Study. American Journal of Clinical Nutrition, 90, 1390-1396.

Hunt, M. G., Momjian, A. J., \& Wong, K. K. (2011). Effects of diurnal variation and caffeine consumption on Test of Variables of Attention (TOVA) performance in healthy young adults. Psychological Assessment, 23, 226-233.

Huxley, R., Lee, C. M., Barzi, F., Timmermeister, L., Czernichow, S., Perkovic, V. et al. (2009). Coffee, decaffeinated coffee, and tea consumption in relation to incident type 2 diabetes mellitus: a systematic review with meta-analysis. Archives of Internal Medicine, 169, 2053-2063.

Ijzerman, H., \& Semin, G. R. (2009). The thermometer of social relations: mapping social proximity on temperature. Psychological Science, 20, 1214-1220.

Isen, A. M. (1999). Positive affect. In T. Dagleish \& M. Power (Eds.), Handbook of Cognition and Emotion (pp. 521-539). Sussex, England: Wiley.

Isen, A. M., Daubman, K. A., \& Nowicki, G. P. (1987). Positive affect facilitates creative problem solving. Journal of Personality and Social Psychology, 52, 1122-1131.

Isen, A. M., Labroo, A. A., \& Durlach, P. (2004). An influence of product and brand name on positive affect: Implicit and explicit measures. Motivation and Emotion, 28, 43-65.

James, J. E. (2004). Critical review of dietary caffeine and blood pressure: A relationship that should be taken more seriously. Psychosomatic Medicine, 66, 63-71.

James, J. E., \& Rogers, P. J. (2005). Effects of caffeine on performance and mood: withdrawal reversal is the most plausible explanation. Psychopharmacology, 182, 1-8.

Jasso, H., \& Triesch, J. (2007). Learning to attend - from bottom-up to top-down. In R.E. Paletta L. (Ed.), Attention in cognitive systems: theories and systems from an interdisciplinary viewpoint (pp. 106122). Berlin, Heidelberg: Springer-Verlag.

Jing, Y., Han, G., Hu, Y., Bi, Y., Li, L., \& Zhu, D. (2009). Tea consumption and risk of type 2 diabetes: a meta-analysis of cohort studies. The Journal of General Internal Medicine, 24, 557-562.

Juneja, L. R., Chu, D. C., Okubi, T., Nagato, Y., \& Yokogoshi, H. (1999). L-theanine - a unique amino acid of green tea and its relaxation effect in humans. Trends in Food Science \& Technology, 10, 199-204.

Kakuda, T., Hinoi, E., Abe, A., Nozawa, A., Ogura, M., \& Yoneda, Y. (2008). Theanine, an ingredient of green tea, inhibits $[3 \mathrm{H}]$ glutamine transport in neurons and astroglia in rat brain. Journal of Neuroscience Research, 86, 1846-1856.

Kakuda, T., Nozawa, A., Sugimoto, A., \& Niino, H. (2002). Inhibition by theanine of binding of [3H]AMPA, $[3 \mathrm{H}]$ kainate, and [3H]MDL 105,519 to glutamate receptors. Bioscience, Biotechnology, and Biochemistry, 66, 2683-2686. 
Kakuda, T., Nozawa, A., Unno, T., Okamura, N., \& Okai, O. (2000). Inhibiting effects of theanine on caffeine stimulation evaluated by EEG in the rat. Bioscience, Biotechnology, and Biochemistry, 64, 287-293.

Keane, M. A., \& James, J. E. (2008). Effects of dietary caffeine on EEG, performance and mood when rested and sleep restricted. Human Psychopharmacology, 23, 669-680.

Kelemen, W. L., \& Creeley, C. E. (2001). Caffeine (4 mg/kg) influences sustained attention and delayed free recall but not memory predictions. Human Psychopharmacology, 16, 309-319.

Kelly, S. P., Gomez-Ramirez, M., Montesi, J. L., \& Foxe, J. J. (2008). L-theanine and caffeine in combination affect human cognition as evidenced by oscillatory alpha-band activity and attention task performance. Journal of Nutrition, 138, 1572S-1577S.

Kenemans, J. L., \& Lorist, M. M. (1995). Caffeine and selective visual processing. Pharmacology, Biochemistry and Behaviour, 52, 461-471.

Kenemans, J. L., \& Verbaten, M. N. (1998). Caffeine and visuo-spatial attention. Psychopharmacology, 135, 353-360.

Kenemans, J. L., Wieleman, J. S., Zeegers, M., \& Verbaten, M. N. (1999). Caffeine and stroop interference. Pharmacology, Biochemistry and Behaviour, 63, 589-598.

Kennedy, D. O., \& Haskell, C. F. (2011). Cerebral blood flow and behavioural effects of caffeine in habitual and non-habitual consumers of caffeine: A near infrared spectroscopy study. Biological Psychology, 86, 298-306.

Ker, K., Edwards, P. J., Felix, L. M., Blackhall, K., \& Roberts, I. (2010). Caffeine for the prevention of injuries and errors in shift workers. Cochrane Database of Systematic Reviews, CD008508.

Kim, A., Chiu, A., Barone, M. K., Avino, D., Wang, F., Coleman, C. I. et al. (2011). Green tea catechins decrease total and low-density lipoprotein cholesterol: a systematic review and meta-analysis. Journal of the American Dietetic Association, 111, 1720-1729.

Kimura, K., Kurita, M., \& Murata, T. (1975). Influence of alkylamides of glutamic acid and related compounds on the central nervous system III. Effect of theanine on spontaneous activity of mice. Chemical \& Pharmaceutical Bulletin, 95, 892-895.

Kimura, K., Ozeki, M., Juneja, L. R., \& Ohira, H. (2007). L-Theanine reduces psychological and physiological stress responses. Biological Psychology, 74, 39-45.

King, S. C., Meiselman, H. L., \& Carr, B. T. (2010). Measuring emotions associated with foods in consumer testing. Food Quality and Preference, 21, 1114-1116.

Klimesch, W., Doppelmayr, M., Russegger, H., Pachinger, T., \& Schwaiger, J. (1998). Induced alpha band power changes in the human EEG and attention. Neuroscience Letters, 244, 76.

Koppelstaetter, F., Poeppel, T. D., Siedentopf, C. M., Ischebeck, A., Kolbitsch, C., Mottaghy, F. M. et al. (2010). Caffeine and cognition in functional magnetic resonance imaging. Journal of Alzheimer's Disease, 20, S71-S84.

Kraiger, K., Billing, R. S., \& Isen, A. M. (1989). The influence of positive affective states on task perceptions and satisfaction. Organizational Behaviour and Human Decision Processes, 44, 12-25.

Larsen, J. T., Berntson, G. G., Poehlmann, K. M., Ito, T. A., \& Cacioppo, J. T. (2008). The psychophysiology of emotion. In M. Lewis, J. M. Haviland-Jones, \& L. Feldman-Barrett (Eds.), Handbook of emotions (pp. 180-195). New York: Guilford Press.

Levin, E. D., Bushnell, P. J., \& Rezvani, A. H. (2011). Attention-modulating effects of cognitive enhancers. Pharmacology, Biochemistry and Behaviour, 99, 154.

Lieberman, H. R. (1992). Caffeine. In A.P. Smith \& D. M. Jones (Eds.), Handbook of Human Performance (2 ed., pp. 49-72). London: Academic Press.

Lieberman, H. R., Wurtman, R. J., Emde, G. G., Roberts, C., \& Coviella, I. L. (1987). The effects of low doses of caffeine on human performance and mood. Psychopharmacology, 92, 308-312.

Lim, J., \& Dinges, D. F. (2010). A meta-analysis of the impact of short-term sleep deprivation on cognitive variables. Psychological Bulletin, 136, 375-389.

Lorist, M. M., \& Tops, M. (2003). Caffeine, fatigue, and cognition. Brain and Cognition, 53, 82-94. 
Lorist, M. M., Snel, J., Kok, A., \& Mulder, G. (1994). Influence of caffeine on selective attention in wellrested and fatigued subjects. Psychophysiology, 31, 525-534.

Lorist, M. M., Snel, J., Kok, A., \& Mulder, G. (1996). Acute effects of caffeine on selective attention and visual search processes. Psychophysiology, 33, 354-361.

Lu, K., Gray, M. A., Oliver, C., Liley, D. T., Harisson, B. J., \& Bartolomeusz, C. F. (2013). The acute effects of L-theanine in comparison with alprazolam on anticipatory anxiety in humans. Human Psychopharmacology, 19, 465 .

Lucas, R. E., Diener, E., \& Larsen, R. J. (2009). Measuring positive emotions. In E. Diener (Ed.), Assessing Well-Being (pp. 139-155) Springer.

Lumme, V., Aalto, S., Ilonen, T., Nagren, K., \& Hietala, J. (2007). Dopamine D2/D3 receptor binding in the anterior cingulate cortex and executive functioning. Psychiatry Research, 156, 69-74.

Lyubomirsky, S., King, L., \& Diener, E. (2005). The benefits of frequent positive affect: does happiness lead to success? Psychological Bulletin, 131, 803-855.

Macfarlane, A., \& Macfarlane, I. (2004). The empire of tea: the remarkable history of the plant that took over the world. New York: Overlook Press.

Macht, M., Meiniger, J., \& Roth, J. (2005). The pleasures of eating: A qualitative analysis. Journal of Happiness Studies, 6, 137-160.

Magkos, F., \& Kavouras, S. A. (2005). Caffeine use in sports, pharmacokinetics in man, and cellular mechanisms of action. Critical Reviews in Food Science and Nutrition, 45, 535-562.

Magkos, F., \& Sidossis, L. S. (2005). Methodological approaches to the study of metabolism across individual tissues in man. Current Opinion in Clinical Nutrition and Metabolic Care, 8, 501-510.

Maridakis, V., Herring, M. P., \& O'Connor, P. J. (2009). Sensitivity to change in cognitive performance and mood measures of energy and fatigue in response to differing doses of caffeine or breakfast. International Journal of Neuroscience, 119, 975-994.

Maridakis, V., O'Connor, P. J., \& Tomporowski, P. D. (2009). Sensitivity to change in cognitive performance and mood measures of energy and fatigue in response to morning caffeine alone or in combination with carbohydrate. International Journal of Neuroscience, 119, 1239-1258.

Martin, F. H., \& Garfield, J. (2006). Combined effects of alcohol and caffeine on the late components of the event-related potential and on reaction time. Biological Psychology, 71, 63-73.

Mauss, I. B., \& Robinson, M. D. (2009). Measures of emotion. Cognition and Emotion, 23, 237.

McLellan, T. M., Kamimori, G. H., Bell, D. G., Smith, I. F., Johnson, D., \& Belenky, G. (2005a). Caffeine maintains vigilance and marksmanship in simulated urban operations with sleep deprivation. Aviation, Space and Environmental Medicine, 76, 39-45.

McLellan, T. M., Kamimori, G. H., Voss, D. M., Bell, D. G., Cole, K. G., \& Johnson, D. (2005b). Caffeine maintains vigilance and improves run times during night operations for Special Forces. Aviation, Space and Environmental Medicine, 76, 647-654.

McLellan, T. M., Kamimori, G. H., Voss, D. M., Tate, C., \& Smith, S. J. (2007). Caffeine effects on physical and cognitive performance during sustained operations. A Aviation, Space and Environmental Medicine, 78, 871-877.

McNair, D. M., Lorr, M., \& Droppleman, L. F. (1992). The Profile of Mood States (POMS) Manual. San Diego, CA: EdITS.

Mednick, S. A. (1962). The associative basis of the creative process. Psychological Review, 69, 220-232.

Mesquita, B., \& Frijda, N. H. (1992). Cultural variations in emotions: a review. Psychological Bulletin, 112, 179-204.

Mets, M. A. J., Baas, D., Van Boven, I., Olivier, B., \& Verster, J. C. (2012). Effects of coffee on driving performance during prolonged simulated highway driving. Psychopharmacology, 222, 337-342.

Miller, E. K., \& Cohen, J. D. (2001). An integrative theory of prefrontal cortex function. Annual Review of Neuroscience, 24, 167-202.

Miner, A., Glomb, T., \& Hulin, C. (2005). Experience sampling mood and its correlates at work. Journal of Occupational and Organizational Psychology, 78, 171-193. 
Monsell, S. (2003). Task switching. Trends in Cognitive Sciences, 7, 134-140.

Mumford, G. K., Evans, S. M., Kaminski, B. J., Preston, K. L., Sannerud, C. A., Silverman, K. et al. (1994). Discriminative stimulus and subjective effects of theobromine and caffeine in humans. Psychopharmacology, 115, 1-8.

Murao, S., Yoto, A., \& Yokogoshi, H. (2013). Effect of smelling green tea on mental status and EEG activity. International Journal of Affective Engineering, 12, 37-43.

Nehlig, A. (2010). Is Caffeine a Cognitive Enhancer? Journal of Alzheimer's Disease, 20, S85-S94.

Nehlig, A., Daval, J. L., \& Debry, G. (1992). Caffeine and the central nervous system: mechanisms of action, biochemical, metabolic and psychostimulant effects. Brain Research Reviews, 17, 139-170.

Ng, T. P., Feng, L., Niti, M., Kua, E. H., \& Yap, K. B. (2008). Tea consumption and cognitive impairment and decline in older Chinese adults. American Journal of Clinical Nutrition, 88, 224-231.

Nijstad, B. A., De Dreu, C. K., Rietzschel, E. F., \& Baas, M. (2010). The dual pathway to creativity model: Creative ideation as a function of flexibility and persistence. European Review of Social Psychology, 21, 34-77.

Niu, K., Hozawa, A., Kuriyama, S., Ebihara, S., Guo, H., Nakaya, N. et al. (2009). Green tea consumption is associated with depressive symptoms in the elderly. American Journal of Clinical Nutrition, 90, 1615-1622.

Nobre, A. C., Rao, A., \& Owen, G. N. (2008). L-theanine, a natural constituent in tea, and its effect on mental state. Asia Pacific Journal of Clinical Nutrition, 17, 167-168.

Nurk, E., Refsum, H., Drevon, C. A., Tell, G. S., Nygaard, H. A., Engedal, K. et al. (2009). Intake of flavonoid-rich wine, tea, and chocolate by elderly men and women is associated with better cognitive test performance. Journal of Nutrition, 139, 120-127.

Owen, G. N., Parnell, H., De Bruin, E. A., \& Rycroft, J. A. (2008). The combined effects of L-theanine and caffeine on cognitive performance and mood. Nutritional Neuroscience, 11, 193-198.

Panza, F., Solfrizzi, V., Barulli, M. R., Bonfiglio, C., Guerra, V., Osella, A. et al. (2015). Coffee, tea, and caffeine consumption and prevention of late-life cognitive decline and dementia: a systematic review. Journal of Nutrition Health and Aging, 19, 313-328.

Peeling, P., \& Dawson, B. (2007). Influence of caffeine ingestion on perceived mood states, concentration, and arousal levels during a 75-min university lecture. Advances in Physiology Education, 31, 332-335.

Pelegrino, D. A., Xu, H., \& Vetri, F. (2010). Caffeine and the Control of Cerebral Hemodynamics. Journal of Alzheimer's Disease, 20, S51-S62.

Pham, N. M., Nanri, A., Kochi, T., Kuwahara, K., Tsuruoka, H., Kurotani, K. et al. (2014). Coffee and green tea consumption is associated with insulin resistance in Japanese adults. Metabolism, 63, 400-408.

Philip, P., Taillard, J., Moore, N., Delord, S., Valtat, C., Sagaspe, P. et al. (2006). The effects of coffee and napping on nighttime highway driving: a randomized trial. Annals of Internal Medicine, 144, 785-791.

Piqueras-Fiszman, B., \& Jaeger, S. R. (2015). The effect of product-context appropriateness on emotion associations in evoked eating occasions. Food Quality and Preference, 40, 49-60.

Piqueras-Fiszman, B., \& Spence, C. (2015). Sensory expectations based on product-extrinsic food cues: an interdisciplinary review of the empirical evidence and theoretical accounts. Food Quality and Preference, 40, 165-179.

Porkka-Heiskanen, T. (1999). Adenosine in sleep and wakefulness. Annals of Medicine, 31, 125-129.

Posner, M. I., \& Dehaene, S. (1994). Attentional networks. Trends in Neurosciences, 17, 75-79.

Posner, M. I., \& Petersen, S. E. (1990). The attention system of the human brain. Annual Review of Neuroscience, $13,25-42$.

Posner, M. I., \& Rothbart, M. K. (2007). Research on attention networks as a model for the integration of Psychological Science. Annual Review of Psychology, 58, 1-23.

Pross, N., Demazieres, A., Girard, N., Barnouin, R, Santoro, F., et al. (2013) Influence of progressive fluid restriction on mood and physiological markers of dehydration in women. British Journal of Nutrition, $109,313-321$. 
Quinlan, P., Lane, J., \& Aspinall, L. (1997). Effects of hot tea, coffee and water ingestion on physiological responses and mood: the role of caffeine, water and beverage type. Psychopharmacology, 134, 164-173.

Quinlan, P. T., Lane, J., Moore, K. L., Aspen, J., Rycroft, J. A., \& O'Brien, D. C. (2000). The acute physiological and mood effects of tea and coffee: the role of caffeine level. Pharmacology, Biochemistry and Behaviour, 66, 19-28.

Quinlan, P., Lane, J., \& Aspinall, L. (1997). Effects of hot tea, coffee and water ingestion on physiological responses and mood: the role of caffeine, water and beverage type. Psychopharmacology, 134, 164-173.

Quirin, M., Kazen, M., Rohrmann, S., \& Kuhl, J. (2009). Implicit but not explicit affectivity predicts circadian and reactive cortisol: using the implicit positive and negative affect test. Journal of Personality, 77 , 401-425.

Ras, R. T., Zock, P. L., \& Draijer, R. (2011). Tea consumption enhances endothelial-dependent vasodilation; a meta-analysis. PLoS One, 6, e16974.

Raz, A., \& Buhle, J. (2006). Typologies of attentional networks. Nature Reviews Neuroscience, 7, 367-379.

Rees, K., Allen, D., \& Lader, M. (1999). The influences of age and caffeine on psychomotor and cognitive function. Psychopharmacology, 145, 181-188.

Reyner, L. A., \& Horne, J. A. (2002). Efficacy of a 'functional energy drink' in counteracting driver sleepiness. Physiology and Behaviour, 75, 331-335.

Richardson, N. J., Rogers, P. J., Elliman, N. A., \& O'Dell, R. J. (1995). Mood and performance effects of caffeine in relation to acute and chronic caffeine deprivation. Pharmacology, Biochemistry and Behaviour, 52, 313-320.

Robbins, T. W., \& Murray, M. M. (2006). Behavioural pharmacology: 40+ years of progress, with a focus on glutamate receptors and cognition. Trends in Pharmacological Science, 27, 148.

Robelin, M., \& Rogers, P. J. (1998). Mood and psychomotor performance effects of the first, but not of subsequent, cup-of-coffee equivalent doses of caffeine consumed after overnight caffeine abstinence. Behavioural Pharmacology, 9, 611-618.

Rogers, P.J., Kainth, A., \& Smit, H.J. (2001) A drink of water can improve or impair mental performance depending on small differences in thirst. Appetite, 36, 57-58.

Rogers, P. J. (2007). Caffeine, mood and mental performance in everyday life. Nutrition Bulletin, 32, 84-89.

Rogers, P. J., \& Dernoncourt, C. (1998). Regular caffeine consumption: a balance of adverse and beneficial effects for mood and psychomotor performance. Pharmacology, Biochemistry and Behaviour, 59, 1039-1045.

Rogers, P. J., Heatherley, S. V., Hayward, R. C., Seers, H. E., Hill, J., \& Kane, M. (2005). Effects of caffeine and caffeine withdrawal on mood and cognitive performance degraded by sleep restriction. Psychopharmacology, 179, 742-752.

Rogers, P. J., Smith, J. E., Heatherley, S. V., \& Pleydell-Pearce, C. W. (2008). Time for tea: mood, blood pressure and cognitive performance effects of caffeine and theanine administered alone and together. Psychopharmacology, 195, 569-577.

Rogers, R. D., \& Monsell, S. (1995). Costs of a predictable switch between simple cognitive tasks. Journal of Experimental Psychology: General, 124, 207-231.

Roozen, H. G., Wiersema, H., Strietman, M., Feij, J. A., Lewinsohn, P. M., Meyers, R. J. et al. (2008). Development and psychometric evaluation of the pleasant activities list. American Journal of Addiction, 17, 422-435.

Rousmans, S., Robin, O., Dittmar, A., \& Vernet-Maury, E. (2000). Autonomic nervous system responses associated with primary tastes. Chemical Senses, 25, 709-718.

Rowe, G., Hirsch, J. B., \& Anderson, A. K. (2007). Positive affect increases the breadth of attentional selection. Proceedings of the National Academy of Sciences, 101, 383-388.

Rozin, P. (1999). Preadaptation and the puzzles and properties of pleasure. In E. Diener \& N. Schwarz (Eds.), Well being: The foundations of hedonic psychology (pp. 109-133). New York: Russel Sage.

Ruijter, J., de Ruiter, M. B., \& Snel, J. (2000a). The effects of caffeine on visual selective attention to color: an ERP study. Psychophysiology, 37, 427-439. 
Ruijter, J., de Ruiter, M. B., Snel, J., \& Lorist, M. M. (2000b). The influence of caffeine on spatial-selective attention: an event-related potential study. Clinical Neurophysiology, 111, 2223-2233.

Ruijter, J., Lorist, M. M., \& Snel, J. (1999). The influence of different doses of caffeine on visual task performance. Journal of Psychophysiology, 13, 37-48.

Ruijter, J., Lorist, M. M., Snel, J., \& de Ruiter, M. B. (2000c). The influence of caffeine on sustained attention: an ERP study. Pharmacology Biochemistry and Behaviour, 66, 29-37.

Russell, J. A. (1989). Affect Grid: A single-item scale of pleasure and arousal. Journal of Personality and Social Psychology, 57, 493-502.

Russell, J. A., \& Carroll, J. M. (1999). On the bipolarity of positive and negative affect. Psychological Bulletin, 125, 3-30.

Ruxton, C. H. S. (2008). The impact of caffeine on mood, cognitive function, performance and hydration: a review of benefits and risks. Nutrition Bulletin, 33, 15-25.

Scheid, L., Ellinger, S., Alteheld, B., Herholz, H., Ellinger, J., \& Henn, T. (2012). Kinetics of L-theanine uptake and metabolism in healthy participants are comparable after ingestion of L-theanine via capsules and green tea. Journal of Nutrition, 209, 6.

Schmitt, J. A., Hogervorst, E., Vuurman, E. F., Jolles, J., \& Riedel, W. J. (2003). Memory functions and focussed attention in middle-aged and elderly subjects are unaffected by a low, acute dose of caffeine. Journal of Nutrition Health and Aging, 7, 301-303.

Scholey, A., Downey, L. A., Ciorciari, J., Pipingas, A., Nolidin, K., Finn, M. et al. (2012). Acute neurocognitive effects of epigallocatechin gallate (EGCG). Appetite, 58, 767-770.

Schwarz, N. (1990). Feelings as information: Informational and motivational functions of affective states. In E.T. Higgins \& R. Sorrentino (Eds.), Handbook of motivation and cognition: Foundations of social behaviour (2 ed., pp. 527-561). New York: Guilford.

Seng, K. Y., Fun, C. Y., Law, Y. L., Lim, W. M., Fan, W., \& Lim, C. L. (2009). Population pharmacokinetics of caffeine in healthy male adults using mixed-effects models. Journal of Clinical Pharmacy and Therapeutics, 34, 103-114.

Serra-Grabulosa, J. M., Adan, A., Falcon, C., \& Bargallo, N. (2010). Glucose and caffeine effects on sustained attention: an exploratory fMRI study. Human Psychopharmacology, 25, 543-552,

Shimbo, M., Nakamura, K., Jing, S. H., Kizuki, M., Seino, K., Inose, T. et al. (2005). Green tea consumption in everyday life and mental health. Public Health Nutrition, 8, 1300-1306.

Sio, U. N., \& Ormerond, T. C. (2009). Does incubation enhance problem solving? A meta-analytic review. Psychological Bulletin, 135, 94-120.

Smit, H. J., \& Rogers, P. J. (2000). Effects of low doses of caffeine on cognitive performance, mood and thirst in low and higher caffeine consumers. Psychopharmacology, 152, 167-173.

Smith, A. (2002). Effects of caffeine on human behaviour. Food and Chemical Toxicology, 40, 1243-1255.

Smith, A. (2009a). Effects of caffeine in chewing gum on mood and attention. Human Psychopharmacology, $24,239-247$.

Smith, A. P. (2005a). Caffeine. In H.R. Lieberman, R. B. Kanarek, \& C. Prasad (Eds.), Nutritional Neuroscience (pp. 335-359). Boca Raton, FL: CRC Press.

Smith, A. P. (2005b). Caffeine at work. Human Psychopharmacology, 20, 441-445.

Smith, A. P. (2009b). Caffeine, cognitive failures and health in a non-working community sample. Human Psychopharmacology, 24, 29-34.

Smith, A. P., Brockman, P., Flynn, R., Maben, A., \& Thomas, M. (1993). Investigation of the effects of coffee on alertness and performance during the day and night. Neuropsychobiology, 27, 217-223.

Smith, A. P., Christopher, G., \& Sutherland, D. (2006). Effects of caffeine in overnight-withdrawn consumers and non-consumers. Nutritional Neuroscience, 9, 63-71.

Smith, A. P., Clark, R., \& Gallagher, J. (1999b). Breakfast cereal and caffeinated coffee: effects on working memory, attention, mood, and cardiovascular function. Physiology and Behaviour, 67, 9-17.

Smith, A. P., Kendrick, A. M., \& Maben, A. L. (1992). Effects of breakfast and caffeine on performance and mood in the late morning and after lunch. Neuropsychobiology, 26, 198-204. 
Smith, A., Brice, C., Nash, J., Rich, N., \& Nutt, D. J. (2003). Caffeine and central noradrenaline: effects on mood, cognitive performance, eye movements and cardiovascular function. Journal of Psychopharmacology, 17, 283-292.

Smith, A., Kendrick, A., Maben, A., \& Salmon, J. (1994a). Effects of breakfast and caffeine on cognitive performance, mood and cardiovascular functioning. Appetite, 22, 39-55.

Smith, A., Maben, A., \& Brockman, P. (1994b). Effects of evening meals and caffeine on cognitive performance, mood and cardiovascular functioning. Appetite, 22, 57-65.

Smith, A., Sturgess, W., \& Gallagher, J. (1999a). Effects of a low dose of caffeine given in different drinks on mood and performance. Human Psychopharmacology Clinical, 14, 473-482.

Smith, A., Sutherland, D., \& Christopher, G. (2005). Effects of repeated doses of caffeine on mood and performance of alert and fatigued volunteers. Journal of Psychopharmacology, 19, 620-626.

Smith, A., Whitney, H., Thomas, M., Perry, K., \& Brockman, P. (1997). Effects of caffeine and noise on mood, performance and cardiovascular functioning. Human Psychopharmacology, 12, 27-33.

Song, J., Xu, H., Liu, F., \& Feng, L. (2012). Tea and cognitive health in late life: current evidence and future directions. The Journal of Nutrition Health and Aging, 16, 31-34.

Spinelli, S., Masi, C., Zoboli, G. P., Prescott, J., \& Monteleane, E. (2015). Emotional responses to branded and unbranded foods. Food Quality and Preference, 42, 1-11.

Stephens, C. L., Christie, I. C., \& Friedman, B. H. (2010). Autonomic specificity of basic emotions: evidence from pattern classification and cluster analysis. Biological Psychology, 84, 463-473.

Steptoe, A., Gibson, E. L., Vuononvirta, R., Williams, E. D., Hamer, M., Rycroft, J. A. et al. (2007). The effects of tea on psychophysiological stress responsivity and post-stress recovery: a randomised double-blind trial. Psychopharmacology, 190, 81-89.

Strayer, D. L., \& Drews, F. A. (2007). Attention. In F. Durso, R. Nickerson, S. Dumains, S. Lewandowsky, \& T. Perdect (Eds.), Handbook of Applied Cognition (Second ed., ). West Sussex, UK: John Wiley \& Sons, Ltd.

Subramaniam, K., Kounios, J., Parrish, T. B., \& Jung-Beeman, M. (2009). A brain mechanism for facilitation of insight by positive affect. Journal of Cognitive Neuroscience, 21, 415-432.

Sunram-Lea, S. I., Owen, L., Finnegan, Y., \& Hu, H. (2011). Dose-response investigation into glucose facilitation of memory performance and mood in healthy young adults. Journal of Psychopharmacology, $25,1076-1087$

Terashima, T., Takido, J., \& Yokogoshi, H. (1999). Time-dependent changes of amino acids in the serum, liver, brain and urine of rats administered with theanine. Bioscience, Biotechnology, and Biochemistry, 63, 615-618.

Tieges, Z., Richard, R. K., Snel, J., \& Kok, A. (2004). Caffeine strengthens action monitoring: evidence from the error-related negativity. Cognitive Brain Research, 21, 87-93.

Tieges, Z., Snel, J., Kok, A., \& Richard, R. K. (2009). Caffeine does not modulate inhibitory control. Brain \& Cognition, 69, 316-327.

Tieges, Z., Snel, J., Kok, A., Plat, N., \& Ridderinkhof, R. (2007). Effects of caffeine on anticipatory control processes: Evidence from a cued task-switch paradigm. Psychophysiology, 44, 561-578.

Tieges, Z., Snel, J., Kok, A., Wijnen, J. G., Lorist, M. M., \& Ridderinkhof, R. (2006). Caffeine improves anticipatory processes in task switching. Biological Psychology, 73, 101-113.

Van Boxtel, M. P., Schmitt, J. A., Bosma, H., \& Jolles, J. (2003). The effects of habitual caffeine use on cognitive change: a longitudinal perspective. Pharmacology, Biochemistry and Behaviour, 75, 921927.

Van der Pijl, P., Chen, L., \& Mulder, T. (2010). Human disposition of L-theanine in tea or aqueous solution. Journal of Functional Foods, 2, 239-244.

Varani, K., Portaluppi, F., Merighi, S., Ongini, E., Belardinelli, L., \& Borea, P. A. (1999). Caffeine alters A2A adenosine receptors and their function in human platelets. Circulation, 99, 2499-2502.

Vohs, K. D., Wang, Y., Gino, F., \& Norton, M. I. (2013). Rituals enhance consumption. Psychological Science, 24, 1714-1721. 
Warburton, D. M. (1995). Effects of caffeine on cognition and mood without caffeine abstinence. Psychopharmacology, 119, 66-70.

Warburton, D. M., Bersellini, E., \& Sweeney, E. (2001). An evaluation of a caffeinated taurine drink on mood, memory and information processing in healthy volunteers without caffeine abstinence. Psychopharmacology, 158, 322-328.

Ward, T. B., Patterson, M. J., \& Sifonis, C. M. (2004). The role of specificity and abstraction in creative idea generation. Creativity Research Journal, 16, 1-9.

Watson, D., Clark, L. A., \& Tellegen, A. (1988). Development and validation of brief measures of positive and negative affect: the PANAS scales. Journal of Personality and Social Psychology, 54, 10631070.

Westenhoefer, J., Bellisle, F., Blundell, J. E., de, V. J., Edwards, D., Kallus, W. et al. (2004). PASSCLAIM-mental state and performance. European Journal of Nutrition, 43, II85-II117.

Wightman, E. L., Haskell, C. F., Forster, J. S., Veasey, R. C., \& Kennedy, D. O. (2012). Epigallocatechin gallate, cerebral blood flow parameters, cognitive performance and mood in healthy humans: a double-blind, placebo-controlled, crossover investigation. Human Psychopharmacology, 27, 177-186.

Williams, L. E., \& Bargh, J. A. (2008). Experiencing physical warmth promotes interpersonal warmth. Science, 322, 606-607.

Winkielman, P., \& Berridge, K. C. (2004). Unconscious emotion. Current Directions in Psychological Science, 13, 120-123.

Yeomans, M. R., Ripley, T., Davies, L. H., Rusted, J. M., \& Rogers, P. J. (2002). Effects of caffeine on performance and mood depend on the level of caffeine abstinence. Psychopharmacology, 164, 241-249.

Yokogoshi, H., Kobayashi, M., Mochizuki, M., \& Terashima, T. (1998). Effect of theanine, rglutamylethylamide, on brain monoamines and striatal dopamine release in conscious rats. Neurochemical Research, 23, 667-673.

Yokogoshi, H., Mochizuki, M., \& Saitoh, K. (1998). Theanine-induced reduction of brain serotonin concentration in rats. Bioscience, Biotechnology, and Biochemistry, 62, 816-817.

Yoto, A., Motoki, M., Murao, S., \& Yokogoshi, H. (2012). Effects of L-theanine or caffeine intake on changes in blood pressure under physical and psychological stresses. Journal of Physiological Anthropology, 31, 28.

Zheng, X. X., Xu, Y. L., Li, S. H., Liu, X. X., Hui, R., \& Huang, X. H. (2011). Green tea intake lowers fasting serum total and LDL cholesterol in adults: a meta-analysis of 14 randomized controlled trials. American Journal of Clinical Nutrition, 94, 601-610. 

Summary 
Across geographies and cultures, people share the belief that consumption of tea, particularly when made from the leaves of the Camellia Sinensis plant, provides mental benefits, such as feeling relaxed or energized, and clarity of mind. The aim of this thesis was to further investigate whether the consumption of tea, and/or specific tea ingredients, can be linked to acute mental benefits, using validated scientific measures.

In Chapter 1, I presented a theoretical framework to characterize the acute mental benefits effects of tea consumption. In this framework, I distinguished two benefit dimensions, namely cognitive or performance-related and affective or mood-related. Research on the cognitive benefits of tea has mainly focused on attention. Attention is an important cognitive process, as it allows the brain to effectively deal with the vast amount of input that is continuously received. As such, attention is an important prerequisite for many cognitive processes, including perception, memory and reasoning. Affective benefits can be divided in feelings of arousal versus relaxation (i.e., the "arousal" dimension), and pleasant versus unpleasant feelings (i.e., the "valence" dimension). Also, I distinguished three post-consumption stages, namely immediately after consumption (5 to 10 minutes), shortly after consumption (10 to 30 minutes), and 30 minutes or more after consumption. I also clarified that the research in this thesis (primarily) focusses on black tea.

Chapter 2 pertains to a detailed literature review of the effects of caffeine consumption on attention performance. We investigated a number of specific hypotheses around the effects of caffeine on attention performance, using the findings from 66 scientific studies. We researched whether caffeine would affect attention in general, or specific attention domains. For this purpose, we characterised attention tasks encountered in these studies according to two taxonomies. The first distinguishes between simple and complex attention, while the second distinguishes three attention domains, namely "alerting" (achieving and maintaining a state of high sensitivity to incoming stimuli), "orienting" (the ability to selectively attend to certain stimuli by directing attention to cued areas, such as left or right, or cued modalities, such as auditory or visual) and "executive control" (the ability to resolve conflict among thoughts, feelings, and responses with conscious processing, such as reasoning and inhibition). In line with its proposed 
mechanism of action, findings confirmed that caffeine affected both simple and complex attention tasks and both alerting and executive function domains, but orienting only to a lesser extent. This was in line with our expectations based on the actions of caffeine in the brain. We further concluded that while effects on attention performance occurred even at low caffeine doses (i.e., similar to one or two cups of tea), there was insufficient evidence for a dose-response relationship. Moreover, there was no conclusive evidence that habitual caffeine users require a greater dose of caffeine in order to experience a performance benefit. Finally, we investigated the role of caffeine deprivation, as typically participants are not allowed to consume caffeine some hours before study participation, but we did not see consistent effects of caffeine withdrawal. Notably, the latter questions were more difficult to answer definitively, due to the considerable amount of variation within caffeine studies. In sum, this review indicated that consumption of caffeine, also in doses like present in tea, can improve attention.

In Chapter 3, we investigated the effects of an iced tea drink containing a combination of theanine and caffeine on attention performance and subjective alertness. The combination of theanine and caffeine is unique to tea and both ingredients are known to have psychoactive properties (i.e., they affect brain activity). The drink contained a relatively low dose of caffeine (similar to two cups of tea) and a higher dose of theanine (similar to five cups of tea). It was compared to a placebo drink, similar in colour and taste but without theanine or caffeine. Findings supported earlier evidence on the effects of theanine in combination with caffeine on a complex attention task, where participants were to switch between different response rules. On another complex attention task, where participants were to orient attention to visual or auditory stimuli, effects were smaller and less consistent. This could suggest that the theanine/ caffeine combination improves executive control and alerting rather than orienting of attention, in line with findings from the caffeine review (Chapter 2). Unlike in previous studies, there was no difference between drinks in the level of subjective alertness experienced after consumption. We cannot conclude on a synergistic effect between theanine and caffeine, or the effect of theanine on its own, as ingredients were not tested in isolation. However, combined with the evidence to date, the current study does suggest that a high dose of theanine combined with caffeine at the level of a single cup of tea, can improve certain aspects of attention. 
In Chapter 4, we reviewed the literature to date on the cognitive and affective benefits of tea, and theanine and caffeine in combination. Cognitive benefits of tea, and theanine and caffeine in combination, have been consistently demonstrated in terms of improved attention and alertness from around 30 minutes post-consumption onwards. Affective benefits beyond alertness, have been less extensively researched until now and findings are inconsistent. While tea consistently improved alertness, some studies reported increased feelings of valence or pleasure after tea consumption compared to water while others did not. However, typically studies focused on the former, not the latter. Moreover, studies to date largely neglected the possibility that mood effects may occur during and/or immediately after consumption, due to the tea consumption experience itself.

Chapter 5 contains the first study to examine affective benefits of tea immediately after consumption. Specifically, we investigated both the valence and arousal dimensions of mood, immediately after the preparation and consumption of tea. We also investigated the effects of tea on creative problem solving, as improved mood has been shown to improve creativity. We divided the study participants in three groups: one group prepared and consumed a cup of tea, others consumed a glass of cold water or were asked to recall a past happy memory, a validated positive affect induction. Immediately after consumption, participants who prepared and consumed the tea experienced a higher valence of mood, as compared to the water group and to a similar extent to the effect of the positive affect induction. At the same time, tea consumption evoked a lower level of arousal compared to the affect induction, potentially indicating more relaxation. We also found an indication that tea consumption and the affect induction led to faster responses for difficult problems in the creative problem solving task, as compared to water. It remains to be disentangled which aspect(s) of tea consumption (e.g. sensory aspects, expectations, preparation) led to the reported changes in valence of mood. This study shows that a simple activity such as the preparation and consumption of a cup of tea, can positively boost mood and thereby may affect behaviour, such as creative problem solving.

Finally, the study described in Chapter 6 further investigated the affective benefits of a single cup of tea, immediately after consumption up to one hour after consumption. We 
divided participants in three groups, namely consumption of tea, water or a placebo tea (coloured and flavoured hot water). Our primary interest was the effect of tea consumption on measures of valence and arousal compared to the other drinks. We also investigated differences in discrete or basic emotions (i.e. happiness and anger), and exploratory implicit measures of mood between the drinks. Immediately after consumption, tea increased valence of mood and reduced arousal as compared to placebo tea, but effects did not remain present over time, i.e. up to 60 minutes after consumption. Also, there were no difference in the experienced discrete emotions for the three drinks. Interestingly, consumption of tea or water increased interest in activities overall and specific types of activities, as compared to placebo tea. Specifically, tea consumption seemed to result in higher interest in creative and domestic activities. In contrast to Chapter 5, differences between tea and water, albeit in the same direction, were not significant. However, the tea in the former study was branded and prepared by participants themselves to their own satisfaction, while the tea in the latter study was unbranded and prepared by staff. Also, in contrast to the studies reviewed in Chapter 4, there was no difference between tea and placebo tea on feelings of alertness over time. However, these earlier studies used larger quantities of tea. We concluded that consumption of a single cup of tea may have subtle affective benefits, mainly on valence of mood. We suspect that these effects will likely increase when consumed in the right context. Finally, we also showed that positive mood from tea consumption can positively affect other outcomes, such as interest in activities.

In Chapter 7 we concluded on our investigations on the acute mental benefits of tea consumption, in the light of the theoretical framework shared in Chapter 1. Consumption of (black) tea has consistent cognitive benefits on attention. These effects, evident from 30 minutes post-consumption, can be linked to the mechanism of tea ingredients caffeine and theanine. Future studies could investigate how the current lab-based findings can be extrapolated to real-life activities which require attention, such as work performance and driving. Tea consumption can also have affective benefits. Effects on alertness and arousal from 30 minutes post-consumption are also rather consistent, and are linked to the effects of tea ingredients theanine and caffeine. Tea may also reduce arousal immediately after consumption, which could be indicative of relaxation. Finally, 
tea consumption has positive immediate effects on valence of mood, but effects over time are less consistent. However, effects seem to depend on a multitude of factors to be further investigated, such as preparation, sensory characteristics and context. We therefore conclude on the basis of this thesis, that there is sufficient evidence for widely shared beliefs in the acute mental benefits of tea consumption. 
Samenvatting 
Het drinken van thee, gemaakt van de Camelia Sinensis plant, wordt gedacht goed te zijn voor lichaam en geest. Zo wordt het drinken van thee geassocieerd met mentale ontspanning, maar ook met alertheid, en helderheid van geest. Het doel van dit proefschrift was om met behulp van gevalideerde wetenschappelijke testen te onderzoeken of de consumptie van thee, en/of specifieke thee ingrediënten, acute mentale effecten kan hebben.

In Hoofdstuk 1 presenteerde ik een theoretisch model om de acute mentale effecten van thee te karakteriseren. Ik onderscheidde hierbij twee dimensies, namelijk cognitieve ofwel prestatie-gerelateerd, en affectieve ofwel stemming-gerelateerd. Het onderzoek naar de cognitieve effecten van thee consumptie richt zich vooral op aandacht. Aandacht is een belangrijk cognitief proces, omdat het menselijk brein slechts een beperkte capaciteit heeft voor het verwerken van alle inkomende informatie. Aandacht speelt daarom een belangrijke rol bij andere cognitieve processen zoals waarnemen, denken, en ons geheugen. Bij onderzoek naar affectieve effecten wordt vaak onderscheid gemaakt tussen gevoelens van opwinding versus ontspanning (ofwel de "arousal" dimensie) en gevoelens van plezierigheid versus onplezierigheid (ofwel de "valentie" dimensie). Ook maakte ik onderscheid tussen drie fasen waarin deze effecten optreden, namelijk onmiddellijk na consumptie ( 5 tot 10 minuten erna), kort na consumptie (10 tot 30 minuten erna) en meer dan een half uur na consumptie. Het onderzoek in dit proefschrift richt zich daarbij (voornamelijk) op zwarte thee.

Hoofdstuk 2 betreft een literatuuronderzoek naar de effecten van cafeïne op aandacht. We formuleerden een aantal specifieke hypothesen, die we vervolgens met behulp van informatie uit 66 gepubliceerde studies onderzochten. Allereerst onderzochten we of cafeïne een algemeen effect heeft op aandacht, of juist specifieke effecten op bepaalde aspecten van aandacht. We deelden de verschillende aandachtstaken gebruikt in de studies op twee manieren in: als een maat van simpele of complexe vormen van aandacht, en volgens een model dat drie verschillende aandachts domeinen onderscheidt, namelijk "alerting" (de constante alertheid om relevante informatie op te kunnen pikken), "orienting" (het richten en verschuiven van aandacht naar bepaalde ruimtelijke posities, zoals links of rechts, of modaliteiten, zoals visueel of auditief) of "executive 
function" (bewuste, gecontroleerde processen, zoals redeneren en inhibitie, om conflicten op te lossen gedachten, gevoelens en responsen). We constateerden dat cafeïne een duidelijk positief effect had op simpele én complexe vormen van aandacht, en op alerting en executive function, maar in mindere mate op orienting. Dit kwam overeen met onze verwachtingen op basis van de werking van cafeïne in het brein. We concludeerden ook dat een lage dosis cafeïne (gelijk aan de dosis in één of twee koppen thee) al een positief effect had op taakprestaties. Er was echter onvoldoende bewijs dat een hogere dosis altijd leidt tot een sterker effect. Verder constateerden we dat het onwaarschijnlijk is dat mensen die gewoonlijk veel cafeïne consumeren, meer cafeïne nodig hebben om een positief effect te ervaren. Tot slot keken we naar de rol van cafeïne deprivatie. Zijn de effecten van cafeïne anders wanneer de proefpersoon gedurende enkele uren voorafgaand aan deelname aan het onderzoek geen cafeïne meer heeft geconsumeerd? We zagen echter geen consistente effecten van cafeïne deprivatie. Overigens, vanwege de grote verscheidenheid in studies waren deze laatste vragen niet definitief te beantwoorden. Dit onderzoek laat zien dat het consumeren van een drank die cafeïne bevat, ook in de hoeveelheid zoals aanwezig in thee, aandacht kan verbeteren.

In Hoofdstuk 3 onderzochten we de cognitieve effecten van een iced tea drankje met toegevoegde theanine en cafeïne. Deze combinatie van theanine en cafeïne is uniek voor thee. Bovendien is er bewijs dat beide ingrediënten psychoactief zijn, dat wil zeggen dat ze de activiteit van het brein kunnen beïnvloeden. Het onderzochte drankje bevatte een relatief lage dosis cafeïne (vergelijkbaar met twee koppen thee), maar een veel hogere dosis theanine (vergelijkbaar met vijf koppen thee). Het effect van dit drankje werd vervolgens vergeleken met een placebo drankje, vergelijkbaar wat betreft kleur en smaak maar zonder cafeïne of theanine. We keken hierbij naar de prestaties op twee verschillende complexe aandachtstaken en naar gevoelens van alertheid. Net als in eerdere studies verbeterde de combinatie van theanine en cafeïne het aantal correcte antwoorden op een aandachtstaak waarbij proefpersonen steeds moesten wisselen van taakopdracht. Effecten op de andere complexe taak, waarbij proefpersonen zich afwisselend moesten oriënteren naar visuele of auditieve stimuli, waren kleiner en minder consistent. Dit komt mogelijk doordat deze taak meer gerelateerd is aan orienting, waarvan we eerder al zagen dat het effect van cafeïne minder duidelijk was (Hoofdstuk 2). In 
tegenstelling tot in eerdere studies, was er geen verschil tussen de twee drankjes wat betreft het opwekken van gevoelens van alertheid. Op basis van deze studie kunnen we geen conclusies trekken over de unieke bijdrage van theanine of cafeïne, omdat ze alleen in combinatie geconsumeerd werden. Maar de resultaten van eerder studies in acht nemend, laat deze studie zien dat een hoge dosis theanine in combinatie met cafeïne aspecten van aandacht kan verbeteren.

Hoofstuk 4 bevat wederom een literatuuronderzoek, nu met betrekking tot cognitieve en affectieve effecten van thee en theanine en cafeïne in combinatie. Wat betreft cognitieve effecten zagen we consistente effecten op aandacht: een consistente verbetering in prestaties op aandachtstaken en gevoelens van alertheid vanaf ongeveer 30 minuten na consumptie. Bovendien bleken deze effecten op te treden na consumptie van thee en theanine en cafeïne in combinatie. We vonden minder studies die de affectieve effecten van thee consumptie onderzochten en de resultaten van deze studies waren bovendien minder consistent. Hoewel thee consistent alertheid en arousal verhoogde, werd de ene keer wel een effect gevonden op valentie en de andere keer niet. Echter, het onderzoek tot nu toe richtte zich ook voornamelijk op arousal, en niet valentie. Bovendien negeerden deze studies de mogelijkheid dat effecten van thee niet enkel door de ingrediënten zoals theanine en cafeïne worden veroorzaakt, maar ook door de ervaring van de consumptie zelf. Ook werd niet onderzocht of dit effect al onmiddellijk na consumptie zou kunnen optreden.

In Hoofdstuk 5 onderzochten we voor het eerst stemming direct na thee consumptie. Specifiek onderzochten we het effect van bereiding en consumptie van thee op valentie en arousal. Ook onderzochten we of deze verbetering in stemming door thee consumptie vervolgens ook een positief effect kon hebben op creatief probleem oplossen. We weten namelijk uit eerder onderzoek dat een verbeterde stemming kan leiden tot verbeterde creativiteit. We verdeelden de proefpersonen in drie groepen: de eerste groep bereidde en dronk een kop thee, de tweede dronk een glas water, en de derde groep werd gevraagd een positieve herinnering op te halen, een gevalideerde methode om een positieve stemming te induceren. De proefpersonen in de thee groep, en in de stemmingsmanipulatie groep, rapporteerden een hogere mate van plezierigheid dan de water groep. 
Proefpersonen in de thee groep rapporteerden minder opwinding, dus meer ontspanning, vergeleken met de stemmingsmanipulatie groep. Verder was er een indicatie dat zowel thee als de manipulatie leidden tot het sneller oplossen van moeilijke problemen in de creativiteitstaak vergeleken met water. Welk(e) aspect(en) van thee consumptie hier verantwoordelijk voor zijn, bijvoorbeeld sensorische aspecten, verwachtingen, of de bereiding, dient verder onderzocht te worden. Deze studie laat echter voor het eerst zien dat een simpele alledaagse activiteit zoals het bereiden en drinken van een kop thee, stemming positief kan beïnvloeden en zelfs gedrag kan beïnvloeden, in dit geval creatief probleem oplossen.

De studie in Hoofdstuk 6 onderzocht de affectieve effecten van een kop thee, direct en tot een uur na consumptie. We verdeelden de proefpersonen in drie groepen, namelijk consumptie van een kop thee, een glas water of een placebo thee (heet water met kleur en smaakstoffen). Allereerst onderzochten de mate van arousal en valentie die proefpersonen rapporteerden na consumptie van de verschillende drankjes. Ook onderzochten we verschillen in de ervaring van een aantal discrete of wel basis emoties (zoals geluk en woede) en impliciete stemming tussen de drankjes. Meteen na consumptie van thee nam de mate van plezierigheid toe en de mate van opwinding af vergeleken met placebo thee. Deze effecten waren echter niet terug te vinden later in de tijd. Er was geen verschil in de ervaren discrete emoties. Tot slot, zagen we ook een positief effect op een impliciete maat van stemming, in dit geval een verhoogde interesse in activiteiten na thee en in sommige gevallen ook water consumptie. In tegenstelling tot in Hoofdstuk 5 was het verschil tussen water en thee op de mate van plezierigheid in deze studie niet significant. In beide studies werden dezelfde theezakjes gebruikt. In de studie in Hoofdstuk 5 werd de thee echter door de proefpersonen zelf bereid en was het merk zichtbaar, terwijl in de laatste studie de thee volgens een standaard recept werd bereid door de onderzoeker en het merk niet zichtbaar was. In tegenstelling tot de studies in Hoofdstuk 4 vonden we geen effect van thee consumptie op de mate van alertheid over tijd. Echter, de studies beschreven in Hoofdstuk 4 gebruikten twee of meer koppen thee terwijl de proefpersonen in deze laatste studie slechts één kop thee dronken. We concludeerden dat consumptie van een kop thee subtiele effecten kan hebben op stemming, maar vooral op plezierigheid. We vermoeden dat deze effecten op plezierigheid groter zullen zijn 
als consumptie in de juiste context plaatsvindt. We lieten ook weer zien dat positieve stemming door thee consumptie ook andere uitkomsten positief kan beïnvloeden, in dit geval interesse in activiteiten.

In Hoofdstuk 7 trekken we algemene conclusies over de acute mentale effecten van thee met betrekking tot het theoretisch model uit Hoofdstuk 1. Consumptie van (zwarte) thee heeft duidelijke en consistente cognitieve effecten op aandacht. Deze effecten worden voornamelijk veroorzaakt door thee ingrediënten theanine en cafeïne en treden op vanaf 30 minuten na consumptie. Toekomstige studies zouden kunnen onderzoeken hoe effecten gevonden in het lab, zich vertalen naar een context in het dagelijks leven waarbij aandacht vereist is, zoals op het werk of in de auto. Consumptie van thee heeft ook affectieve effecten. Wat betreft alertheid zijn deze effecten tamelijk consistent. Net als de cognitieve effecten zijn ze gerelateerd aan de werking van theanine en cafeïne en treden ook op vanaf 30 minuten na consumptie. Onmiddellijk na consumptie van één kop thee vonden we echter ook een afname in arousal, wat kan duiden op meer ontspanning. Wat betreft valentie of plezierigheid zijn effecten minder consistent. Meteen na consumptie van thee zien we een toename in valentie, maar de effecten op stemming over tijd moeten nog verder onderzocht worden. Ook moeten de verschillende factoren die mogelijk een rol spelen bij deze effecten, zoals sensorische aspecten, verwachtingen of de bereiding van de thee, verder onderzocht worden. Tot slot kunnen we op basis van dit proefschrift concluderen dat er wel degelijk bewijs is voor de acute mentale effecten van thee, die sinds lange tijd verondersteld worden. 
Valorisation Addendum 
Across geographies and cultures, people seem to share the belief that consumption of tea can deliver mental benefits, and may choose to consume a cup of tea when in need for these benefits. The objective of this thesis was to investigate whether there is any truth to these common beliefs; i.e., to further investigate whether the consumption of tea, and/or specific tea ingredients, can be linked to acute mental benefits, specifically cognitive and affective benefits, using validated scientific measures. In this addendum, I discuss the relevance of the current findings, the key target audiences to which findings are of interest, and describe a number of applications.

\section{Relevance}

The acute mental benefits of tea are highly relevant in this day and age of high cognitive and emotional demand. People constantly seek for things that make them feel and perform better, for example in terms of job performance, managing work-life balance and social commitments.

\section{Cognitive benefits}

As seen in Chapters 2 to $\mathbf{4}$, the cognitive benefits of tea consumption predominantly manifest themselves in terms of improved attention. The human brain has limited information processing capacity. Therefore, our brain is unable to deal with all the input that is continuously received externally, through its sensory systems (e.g., vision, audition) and internally, through cognitive processes (e.g., memory, reasoning). Attention is an essential aspect of cognitive functioning, because it allows our brains to effectively deal with the continuous and complex input in spite of our limited capacity to process information. As such, it is an important prerequisite for many other cognitive processes, such as memory and reasoning. Consequently, when attention is disrupted, this has profound consequences for everyday functioning. As such, the benefits of tea consumption, and consumption of theanine in combination with caffeine, on attention as seen in this thesis are relevant in the very broad context of everyday cognitive functioning. 


\section{Affective benefits}

Moreover, for the first time, in this thesis tea consumption has been shown to generate immediate short-term and subtle improvements in mood. As indicated in Chapter $\mathbf{5}$ and 6, these findings are of importance not only to the research on the benefits of tea, but also to the research on (inductions of) positive mood in general. Improvement in mood have been related to longer-term well-being, motivational and cognitive benefits (e.g., Fredrickson, 2001; Lyubomirsky, King, \& Diener, 2005). Also, positive affect has been shown to improve cognitive processes such as global information processing (Clore \& Huntsinger, 2007) and creativity (Baas, De Dreu, \& Nijstad, 2008). Thus, while simple everyday activities such as tea preparation and consumption may improve mood, this may also in turn affect cognitive processes, via these improvements in mood.

\section{Target audience}

Findings can be relevant to different audiences, as explained below. Firstly and most obviously, findings are relevant to scientists researching the mental benefits of tea and tea ingredients. This thesis brings together some of the previous work in the form of two reviews, confirming that the consumption of black tea (ingredients) has consistent benefits for attention performance (Chapters 2 and 4). Moreover, we conducted a study to replicate and extend effects of a combination of theanine of caffeine on attention (Chapter 5), and two studies to explore immediate effects on mood, which were to date not yet researched (Chapter 5 and 6). Moreover, by demonstrating benefits that stretch beyond attention and subjective alertness, and beyond an ingredient-driven mechanism, this thesis opens up novel opportunities for research, particularly around the consumption experience and the context of tea consumption, in relation to mood.

Secondly, the findings are generally relevant to researchers who investigate the effects of food and beverages consumption, particularly with respect to affective benefits. Many people report to eat, drink, or smoke to feel good (Bennet, 1988), but researchers have struggled to find ways to support some of these effects scientifically. Immediate effects of palatable foods on the reduction of negative feelings are well established (Köster \& Mojet, 2015). With regard to beverages, researchers typically assessed emotional responses in relation to novel drinks (i.e. Kuenzel, Barton, Blanchette, 
Zandstra, Thomas, \& El-Deredy, 2010; Orjuela-Palacio, Zamora, \& Lanari, 2014) or different versions of a similar drink (Gutjar, de Graaf, Kooijman, de Wijk, Nys, ter Horst et al, 2014), rather than emotional responses to familiar and common beverages, such as tea. In line with the model set-forth by Desmet and Schifferstein (2008), our research demonstrates the importance of different sources of food emotions (i.e. sensory attributes, experienced consequences, anticipated consequences, personal or cultural meaning, and actions of associated agents), and the need to further investigate these in the context of consumption.

Thirdly, findings presented in this thesis are relevant to scientists who investigate ways of inducing positive affect, and the benefits thereof. Positive affect can be induced by listening to music, recalling happy memories, moment of gratitude, and the research presented in Chapter 5 and $\mathbf{6}$ of this thesis adds the simple everyday activity of drinking a cup of tea to that list. Moreover, increasing positive affect has been shown to promote positive, favorable characteristics, motivations and behaviours, and build enduring personal and cognitive resources (Lyubomirsky, King, \& Diener, 2005; Fredrickson, 2001). Hence, it is of interest that the improvements in mood seen after consumption of a cup of tea may lead to immediate behavioural and motivational benefits, such as improved creativity and interest.

In addition to the scientific relevance of the research questions, its impact also extends beyond academia. Firstly, findings are highly relevant to (fast moving consumer goods) companies that produce and market tea and tea-based beverages. As such, these findings are relevant to Unilever, which markets tea and tea-based beverages under a number of brands, the biggest being the Lipton ${ }^{\circledR}$ brand. Last but certainly not least, findings are also of relevance to tea consumers across the world. These consumers may already see tea as their beverage of choice, whether or not due to experience with its mental benefits, and feel comforted in their choice by these findings. Or, they may choose to drink a cup of tea more often now, knowing that it could give rise to some much sought after benefits in a natural way. 


\section{Application}

We can distinguish a number of concrete application activities with regard to the different findings from this thesis, in terms of communication (i.e., to the scientific community, to health influencers and/or to consumers) but also in terms of product development and improvement.

\section{Scientific communication}

Naturally, publication of the work reported in this thesis increases the scope of the scientific evidence on mental benefits of (black) tea consumption, as well as the credibility of earlier work in this area. Moreover, it enables strategic opportunities to communicate research findings to key scientific audiences at conferences and in direct interactions. Finally, it may also stimulate further research into this specific scientific area.

\section{Communication to health care professionals}

These findings can also be used for communication to health care professionals, in order to increase their knowledge of tea and its potential benefits. Unilever's health care professional program, for example, includes the communication of work from Unilever researchers as well as affiliated researchers, on benefits of tea and tea ingredient consumption for health, mood and wellbeing, and performance, through symposia, newsletters and direct interactions.

Work reported in this thesis has been used for such communication purposes. For example, the research in Chapter 3 was presented during the $4^{\text {th }}$ Tea Science Symposium (Jakarta, Indonesia in 2010), organised by Unilever. Also, during the $5^{\text {th }}$ International Symposium on Tea \& Human Health (Washington DC, USA in 2012) sponsored by Unilever, we presented the outcomes of the review of studies published as Chapter 4. Moreover, the research reported in Chapter $\mathbf{4}$ and $\mathbf{5}$ has been included in Unilever newsletters on the topics of "Scientific update on tea and attention" (2012), and "Scientific update on the psychological benefits of black tea" (2013). 


\section{Communication to consumers}

Finally, research from this thesis was and will continue to be used for consumeroriented communication, either directly via on-product communication or advertising, or indirectly via the media.

The findings reported in Chapter 4 for example, were presented during a media event at the Food \& Nutrition Conference \& Expo (FNCE; Houston, USA in 2013). Here, Lipton ${ }^{\circledR}$ hosted a 1-hour session with journalists and bloggers, presenting scientific evidence to support the launch of a new product (Lipton ${ }^{\circledR}$ Natural Energy tea). Also, the research in Chapter $\mathbf{5}$ has been actively communicated via Unilever press releases linked to the scientific publications. Finally, on other occasions, publications where deemed relevant by the press, resulting in popular media coverage of our work, i.e., the publications shared in Chapter $\mathbf{3}$ and $\mathbf{4}$.

\section{Product development}

Finally, the research presented in this thesis could also leverage innovative opportunities to increase the cognitive and affective benefits from tea consumption, by optimizing the key contributing factors.

\section{Conclusions}

The findings presented in this thesis show clear relevance. It shows that consumption of a relatively common product such as black tea, can generate benefits for attention and mood, as well as taking initial steps in understanding what such mood benefits could potentially mean for subsequent behavior and performance. Moreover, these findings are relevant to a number of different audiences, both scientific and commercial. Finally, findings have been and will continue to be applied in order to stimulate communication, research and product development activities for (black) tea. 


\section{ADDITIONAL REFERENCES}

Kuenzel, J., Barton, C., Blanchette, I., Zandstra, E., Thomas, A., \& El-Deredy, W. (2010). Affective expectations about food produce a placebo effect and influence liking. Appetite, 55, 167-168.

Köster, E. P., \& Mojet, J. (2015). From mood to food and from food to mood: A psychological perspective on the measurement of food-related emotions in consumer research. Food Research International, 76, $180-191$.

Orjuela-Palacio, J. M., Zamora, M. C., \& Lanari, M. C. (2014). Consumers' acceptance of a high-polyphenol yerba mate/black currant beverage: Effect of repeated tasting. Food Research International, 57, 2633. 

Dank | Thanks 
Het begon allemaal in het voorjaar van 2007. Gedurende drie maanden deed ik onderzoek bij Unilever R\&D Colworth. De wet van Murphy was zeker van toepassing, maar deze leuke en leerzame tijd was ook het startsein voor dit proefschrift. Na mijn eerste publicatie in 2010, heb ik hier naar toegewerkt, al het was het eindpunt niet altijd duidelijk in zicht. Maar ik ben er! En er zijn veel mensen die ik wil bedanken voor de weg ernaartoe.

Allereerst bij voorbaat ontzettend bedankt aan iedereen die op enige wijze verbonden is met dit proefschrift, de onderzoeken zelf, of de publicaties ervan.

I would like to thank my Unilever colleagues, in Vlaardingen, Colworth and elsewhere, with special thanks to the CPB-"family". Everyone who provided input, asked questions, helped me on my way, showed interest or cheered me up, it was all very welcome! Also a big thank you for everything that I have learned in the past $10+$ years.

Rob, Ilse, Chantalle, Liesbeth, bedankt voor jullie gezelligheid op werk en privé (Plein!). Marina, Vanessa, Arno, het is fijn werken bij Refreshment met jullie. Patricia, jij ook hoor! Gail, thank you for introducing me in the world of tea research. Eveline, jij stimuleerde me bij het schrijven van mijn eerste artikel, en ik bleek het nog leuk te vinden ook. Matt, thank you for the statistical support and your sense of humor! Jane, you were involved from those first studies, thank you for your support. René, jij hebt me steeds aangemoedigd, en uiteindelijk begon ik erin te geloven! Ik herinner me nog goed de gesprekken over het cafeïne review en je tips, want het wilde maar niet opschieten.

Annemie, dank voor je hulp in dit proces. Mijn promotoren, zonder jullie had ik hier niet gestaan! Jan, bedankt dat je mijn promotor wilde zijn. Ik vond het leuk dat we samen hebben kunnen werken aan het laatste artikel. Heel erg bedankt voor je input. Timo, jij zorgde dat ik doorzette en hebt me als geen ander geholpen hier te komen. Ontzettend bedankt voor je steun, je ideeën, je tips en dat je altijd zo snel en grondig was. Honderdduizend versies van alles heb je gelezen. We hebben gelukkig ook veel gelachen.

Beste leescommissie, hartelijk bedankt voor het lezen en beoordelen van mijn proefschrift. Het is een eer dat jullie bereid waren om betrokken te zijn bij mijn promotie. Dear Louise, I am honoured that you are part of my committee, thank you for your efforts and for your support. I look forward to meeting you in person. 
Lieve vrienden en familie, dichtbij of verder weg. jullie vonden het allemaal maar wat spannend, zeker aan het eind. Iedere ontmoeting vroegen jullie hoe het ermee stond, zelfs tijdens de Vasteloavend. Bedankt voor jullie enthousiasme en interesse, maar vooral voor jullie vriendschap, steun en de nodige afleiding! Mijn lieve vriendinnen sinds jaar en dag, Chantal, Loes, Miriam, Mijntje, Noor, Verenice, Kyra, we zien elkaar niet zo vaak meer maar het is altijd feest met jullie! Ik ben ook heel blij met de fijne mensen die de afgelopen jaren in mijn leven zijn gekomen in Den Haag. Een speciaal bedankje voor Maaike, voor het zo mooi mogelijk maken van dit boek en de uitnodigingen! Dear friends, near or far, thanks for your interest, support and distraction.

Oma Mia, ik vind het zo leuk dat jij hier bij bent, je leeft altijd zo met me mee!

Paranimfen, Sanne en Jeanine, ik ben super blij dat jullie naast me staan. Naast dat jullie fantastische vriendinnen zijn (ook feest!), weet ik zeker dat jullie me de beste peptalk(s) geven als ik die nodig heb. Bedankt ook voor jullie hulp met voorbereiden (Haagse Kroegentocht), en het meedenken en meelezen.

Pap, mam, Laura en Barbara, het is heel erg fijn dat ik uit zo'n warm en gezellig nest kom. Ik weet dat jullie in me geloven en me steunen, in alles wat ik doe en wat er ook gebeurt. Jullie waren altijd weer trots als ik iets gepubliceerd had of ergens mocht presenteren. Bedankt voor alles!

Jeroen, jij was er ook opeens dat voorjaar. Vanaf het begin riep jij dat ik hiervoor moest gaan en er is zoveel waar ik je voor wil bedanken nu het af is. Dat je altijd meedenkt, me steunt maar ook uitdaagt, presentaties die je hebt aangehoord en stukken die je hebt gelezen. Voor de fantastische avonturen die we hebben beleefd en gewoon voor wie je bent.

De illustratie op de cover is van Stefano Messina, hij zei erover het volgende: "I made this thinking about my partner. She is a huge fan of tea and drinks it when she wants to relax. She says that it's a moment where thoughts go away and she is quiet." Stefano, thank you for allowing me to use this beautiful image! 

Publications 


\section{Peer-reviewed publications}

Einöther, S.J.L., Baas, M., Rowson, M., Giesbrecht, T. (2015). Investigating the effects of tea, water and a positive affect induction on mood and creativity. Food Quality and Preference, 39, 56-61.

Einöther, S.J.L., Martens V.E.G (2013). Benefits of tea consumption for attention, mood, and performance. The American Journal of Clinical Nutrition, 98, 1700S1708 S.

Sathyanarayanan, V., Thomas, T., Einöther, S.J.L., Dobriyal, R., Joshi, M.K., Srinivasan, K. (2013). Brahmi for the better? New findings challenging cognition and anti anxiety effects of Brahmi (Bacopa Monniera) in healthy adults. Psychopharmacology, 227, 299-306.

Einöther S.J.L., Giesbrecht, T. (2013). Caffeine as an attention enhancer: reviewing existing assumptions. Psychopharmacology, 225, 251-274.

Bryan, J, Tuckey, M., Einöther S.J.L., Garczarek, U., Garrick, A., \& De Bruin, E.A. (2012). The relationship between tea and other beverage consumption, work performance and mood. Appetite, 58, 339-346.

Einöther S.J.L., Rycroft J.A., Martens V.E.G., De Bruin E.A (2010). L-Theanine and caffeine improve task switching but not intersensory attention or subjective alertness. Appetite, 54, 406-409.

\section{Book chapters}

Einöther S.J.L., Giesbrecht, T. De Bruin,E.A., Van Buren, L. Van der Pijl, P.C., \& Walden, C.M. (2012). Attention benefits of tea and tea ingredients: a review of the research to date. In: "Tea in Health and Disease Prevention" (V.R. Preedy, Eds.) Elsevier. 


\section{Publications in preparation}

Einöther, S.J.L., Rowson, M., Ramaekers, J.G., Giesbrecht, T. (Submitted). Infusing pleasure: Mood effects of the consumption of a single cup of tea.

Calder, P.C., Boobis, A., Braun, D., Champ, C.L., Dye, L., Einöther, S, Greyling, A., Matthys, C. Putz, P. Wopereis, S., Woodside, J.V., \& Antoine, J. (Submitted). Improving selection of markers in nutrition research: evaluation of the criteria proposed by the ILSI Europe Marker Validation Initiative.

\section{Conference abstracts}

Einöther, S.J.L. (2012). Tea and cognitive function. Abstract and presentation at the 5th International Symposium on Tea \& Health, September 18 2012, in Washington DC, USA.

Einöther, S.J.L., Baas, M., Rowson, M., Giesbrecht, T. (2012). A cup of creativity? Positive affect and insights after tea consumption. Abstract and presentation at the European Conference of Positive Psychology (ECCP), June 26-28 2012, in Moscow, Russia.

Einöther, S.J.L. (2010). L-theanine \& caffeine and attention: Your daily cup of attention. Presentation at the 4th Tea Science Symposium, July 13 2010, Jakarta, Indonesia.

Einöther, S.J.L., Rycroft J.A., Martens V.E.G., De Bruin E.A. (2009). L-theanine and caffeine improve task switching but not intersensory attention or subjective. Abstract and poster presentation at the European Society for Cognitive Psychology (ESCoP) Annual Conference, September 3-6 2009, in Krakow, Poland. 

Curriculum Vitae 
Suzanne Einöther was born on 27 July 1982 in Brunssum, the Netherlands.

She graduated from secondary school at Serviam College in Sittard in 2000. In 2005, she obtained her Master's degree in (Cognitive) Psychology from Maastricht University. During her studies, she spent three months as an exchange student at the Faculty of Psychology at Dalhousie University in Halifax, Canada. She completed her master thesis at Maastricht University on the determinants of risky sexual behaviour in Moroccan adolescents and young adults in the Netherlands. In 2005, she also completed a 3 month internship at Unilever Research \& Development in Vlaardingen.

Since September 2005, Suzanne works as a scientist at Unilever Research \& Development in Vlaardingen. Initially, she was involved in research aimed at generating insights into perception of products and behaviour change. Since 2008, she manages internal and external research on the effects of food and food ingredients on human cognitive performance, mood and well-being.

In 2015, Suzanne was selected to attend the European Nutrition Leadership Platform (ENLP), and in 2016 she was selected to return to the ENLP programme as junior faculty. 
